\title{
ULtRASOUND MEDIATED STUDY OF THE ELECTROKINETIC EFFECTS IN BIOLOGICAL TISSUES AND PHANTOMS
}

\author{
by \\ Aditya Pandya \\ Bachelor of Biomedical Engineering, Gujarat University, Ahmedabad, Gujarat, India, \\ 2008 \\ A thesis \\ presented to Ryerson University \\ in partial fulfillment of the \\ requirements for the degree of \\ Master of Science \\ in the Program of \\ Biomedical Physics \\ Toronto, Ontario, Canada, 2012 \\ (C)Aditya Pandya 2012
}


I hereby declare that I am the sole author of this thesis. This is a true copy of the thesis, including any required final revisions, as accepted by my examiners.

I authorize Ryerson University to lend this thesis to other institutions or individuals for the purpose of scholarly research.

I further authorize Ryerson University to reproduce this thesis by photocopying or by other means, in total or in part, at the request of other institutions or individuals for the purpose of scholarly research.

I understand that my thesis may be made electronically available to the public 
Ultrasound mediated study of the electrokinetic effects in biological tissues and phantoms

Master of Science 2012

Aditya Pandya

Biomedical Physics

Ryerson University

\begin{abstract}
Traditional ultrasound imaging monitors the backscattering properties of the object being imaged. Many hybrid imaging techniques such as photo-acoustic imaging, electro-acoustic imaging etc. have been developed to enhance the contrast in imaging. The main objective of this study was to monitor the electrically induced mechanical changes(EIMC) in ultrasound phantoms. Changes in time shifting of the signals and amplitude changes were investigated. Images were formed using a linear array ultrasound probe based on the time-delay estimates and the amplitude changes occurring in the tissue/phantom structure. EIMC in phantoms were found to depend on the applied electric field, gelling agent concentration, and salt concentration. The dependence of EIMC on the various parameters can be explained by the stiffness and fixed charge densities (FCD) of the phantoms. Knowledge gained from experimental results and its future extensions might culminate to form a method to image tissues based on their electrokinetic properties.
\end{abstract}




\section{Acknowledgements}

I would like to thank Dr. Yuan Xu for believing in me and giving me a chance to pursue this study. His guidance and forethought were very helpful in order to complete this study. I would also like to thank my family members, who were understanding and patient with me during the course of my study. I am grateful to all of my friends who helped and supported me. I would also like to thank Ozkan Doganay and Arthur Worthington for their assistance.

I would give special thanks to Dr. Richard Cobbold and Dr. Jahan Tavakkoli for being a part of my supervisory committee and providing me with their esteemed guidance. I am also very grateful to Dr. Tavakkoli and Dr. Kolios as they lent Sonix RP ultrasound scanner for some of my experiments.

This research was supported by a Discovery grant from Natural Sciences and Engineering Research Council of Canada (NSERC). 


\section{Dedication}

I dedicate this theses to my parents without whom I would not be able to pursue this study. 


\section{Contents}

\begin{tabular}{lll}
\hline & Introduction & 1
\end{tabular}

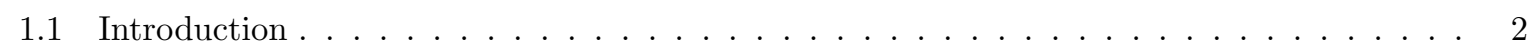

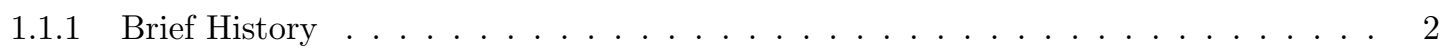

1.1 .2 Motivation . . . . . . . . . . . . . . . . . . . . . . 2

1.1 .3 Organisation of chapters $\ldots \ldots \ldots \ldots \ldots \ldots \ldots$

\begin{tabular}{|ll|l}
\hline & Literature Review & 5
\end{tabular}

2.1 Tissue-mimicking Phantoms used in ultrasound studies $\ldots \ldots \ldots \ldots$

2.1 .1 Gelatin . . . . . . . . . . . . . . . . . . . . . 6

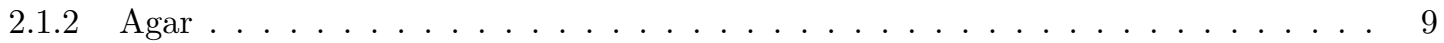

2.2 Conductivity of saline solution $\ldots \ldots \ldots \ldots \ldots \ldots \ldots \ldots$

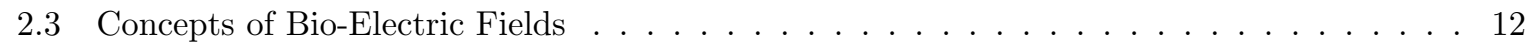

$2.4 \quad$ Exogenic electric fields in tissues $\ldots \ldots \ldots \ldots \ldots$

$2.5 \quad$ Applying $\mathrm{AC}$ and $\mathrm{DC}$ electric fields to biological tissues $\ldots \ldots \ldots \ldots \ldots \ldots$

2.6 Ultra-low frequency/DC and the importance of interstitium $\ldots \ldots \ldots \ldots \ldots$

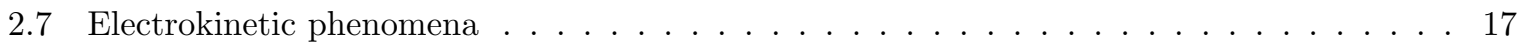

2.8 Recent progress in the area $\ldots \ldots \ldots \ldots \ldots \ldots \ldots$

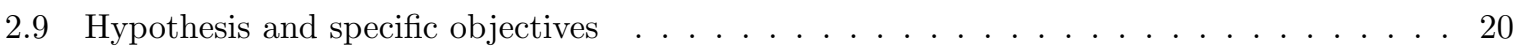

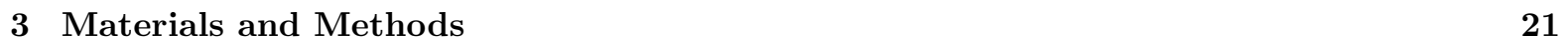

3.1 Important terms used in this chapter . . . . . . . . . . . . . . . . . . . . . 22

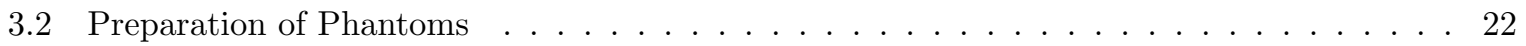

3.3 Experiments including single element transducers $\ldots \ldots \ldots \ldots \ldots \ldots \ldots \ldots$

$3.3 .1 \quad$ Brief description of the algorithm . . . . . . . . . . . . . . . . . 25

3.4 Experiments with Linear Array Transducers $\ldots \ldots \ldots \ldots \ldots \ldots$

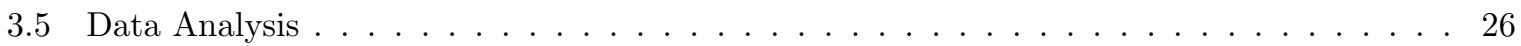

3.5 .1 Observing the changes in slow-time within various windows $\ldots \ldots \ldots$. . . 26

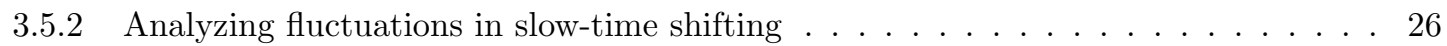

$3.5 .3 \quad$ Evaluating the strain generated . . . . . . . . . . . . . . . . . . . . . . . 29

3.5 .4 Overlay for B-mode images $\ldots \ldots \ldots \ldots \ldots$ 
\begin{tabular}{lll}
\hline 4 & Results & 31
\end{tabular}

4.1 General presentation of the results $\ldots \ldots \ldots \ldots \ldots \ldots \ldots$

4.1 .1 Phantom Nomenclature . . . . . . . . . . . . . . . . . . . . . . . . . . . 32

4.1 .2 Gelatin Phantoms . . . . . . . . . . . . . . . . . . . . . . 32

4.1 .3 Agar Phantoms $\ldots \ldots \ldots \ldots \ldots$

4.2 Fast-time shifting, slow-time shifting and the amplitude changes occurring within the signals 39

$4.2 .1 \quad$ Fast-time signal shifting comparison - different instances in slow time . . . . . . . 39

4.2 .2 Slow-time signal shifting comparison - different windows within the same signal . . 41

$4.2 .3 \quad$ Amplitude changes due to the application of electric fields . . . . . . . . . . . 43

4.3 Dependence of the EIMC on various experimental parameters . . . . . . . . . . . . . 45

$4.3 .1 \quad$ Dependence on the applied electric fields . . . . . . . . . . . . . . . 45

4.3 .2 Dependence on the concentration of gelling agents $\ldots \ldots \ldots \ldots \ldots$

$4.3 .3 \quad$ Dependence on salt concentration . . . . . . . . . . . . . . . . . 53

$4.3 .4 \quad$ Comment on temperature changes during the experimental procedure . . . . . . . 53

4.4 Experiments with a linear array transducer to generate images based on EK effects. . . . 54

\begin{tabular}{|lll}
5 & Discussion and Conclusions & 62
\end{tabular}

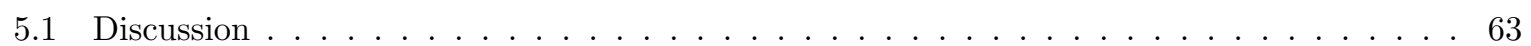

5.2 Conclusions and Future-work $\ldots \ldots \ldots \ldots \ldots \ldots$

\begin{tabular}{ll}
\hline References & 72
\end{tabular} 


\section{List of Tables}

$1.1 \quad$ Applications of electric fields to biological tissues/cells $[12] \ldots \ldots \ldots$

$2.1 \quad$ Some gelling agents used for preparing ultrasound phantoms [24] $\ldots \ldots \ldots \ldots$

$2.2 \quad$ A collection of the response of tissues/cells to electric fields $\ldots \ldots \ldots \ldots$

4.1 Phantom nomenclature and contents . . . . . . . . . . . . . . . . . . . 32

4.2 Temperature measurements in Phantoms $\ldots \ldots \ldots \ldots \ldots \ldots \ldots$ 


\section{List of Figures}

2.1 Microscopic images of (a) 2\% gelatin gel and (b) $10 \%$ gelatin gel. The white bar on the lower right corner represents $100 \mathrm{~nm}[32] \ldots \ldots \ldots \ldots \ldots$

2.2 Elastic modulus of gelatin as a function of concentration extracted from [36] $\ldots \ldots \ldots$

2.3 A plot displaying the ratio of $G^{\prime} / c^{2}$ vs ageing time in hrs. Gelatin weight $\square-1.00 \% \nabla-$ $1.95 \% \triangle-3.00 \% \bigcirc-4.00 \% \bigcirc-5.00 \%$ extracted from $[29] \ldots \ldots \ldots \ldots \ldots$

2.4 The dependence of $G^{\prime}(10 \mathrm{rad} / \mathrm{s})$ on the concentration of gelatin and agar (extracted from[42]) 11

2.5 Conductivity of various concentrations of saline [43] $\ldots \ldots \ldots \ldots \ldots \ldots$

2.6 Schematic representation of a cylindrical conductor $\ldots \ldots \ldots \ldots \ldots$

2.7 Flow of high frequency vs low frequency current through tissues (a schematic). The arrangement represents cell (blue ovals) and the extracellular matrix(white space around

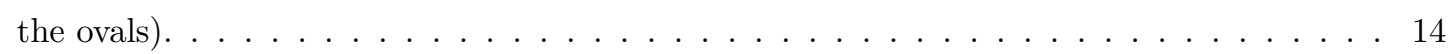

2.8 A schematic of macroscopic anisotropies, extracted from [44]. Layers such as fat, muscles, and bones form anisotropies on a macro-scale. . . . . . . . . . . . . . 15

2.9 A schematic of microscopic anisotropies, extracted from [44]. Microscopic arrangement of cells is responsible for the microscopic anisotropies. . . . . . . . . . . . . . 15

2.10 A simple circuit describing the capacitance of the cell membrane $\left(X_{c}\right)$, Resistance of the intracellular components $\left(R_{I C}\right)$, Resistance of extracellular components $\left(R_{E C}\right) \ldots \ldots$. . . 15

2.11 A pictorial depiction of the interstitium $[53] \ldots \ldots \ldots \ldots \ldots$

2.12 Schematic describing a primitive Electrical double layer $\ldots \ldots \ldots$. . . . . . . 18

2.13 A detailed schematic of an electrical double layer formed near the surface of an electrolyte

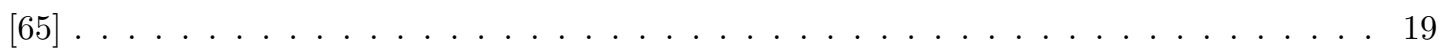

3.1 Experimental setup for single element transducer experiments . . . . . . . . . . . . . . 23

3.2 Experimental procedure for single element transducer experiments . . . . . . . . . . . . . 24

3.3 Description of algorithm being used $\ldots \ldots \ldots \ldots \ldots \ldots \ldots \ldots$

3.4 Experimental procedure for linear array transducer experiments . . . . . . . . . . . . 25

3.5 Procedure for analysing B-mode images $\ldots \ldots \ldots \ldots \ldots \ldots \ldots$

$3.6 \quad$ A schematic for analysing the slow time shifting $\ldots \ldots \ldots \ldots \ldots \ldots$

3.7 A schematic for analysing strain $\ldots \ldots \ldots \ldots \ldots \ldots \ldots \ldots$

3.8 Generating B-mode \& TDE overlays $\ldots \ldots \ldots \ldots$ 
4.1 Conductivity of various concentrations of $\mathrm{NaCl}$ in gelatin phantoms-experimental results.

4.2 (a) Ultrasound A-line as recorded from the phantom G-I. (b) Shifting of the front and the rear boundaries of the phantom G-I during the slow time (black lines represent application of voltage across the phantom). (c) Shifting of the whole phantom G-I from the front to the rear boundary of the phantom at the end of the electric field application. (d) Strain generated due to motion of the phantom G-I during the application of current. . . . . . . 34

4.3 (a) Ultrasound A-line as recorded from phantom G-II. (b) Shifting of the front and the rear boundaries of the phantom G-II during the slow time(black lines represent application of voltage across the phantom). (c) Shifting of the whole phantom G-II from the front to the rear boundary of the phantom at the end of the electric field application. (d) Strain generated due to motion of the phantom G-II during the application of current. . . . . . 35

4.4 (a) Ultrasound A-line as recorded from phantom G- III. (b) Shifting of the front and the rear boundaries of the phantom G-III during the slow time (black lines represent application of voltage across the phantom). (c) Shifting of the whole phantom G-III from the front to the rear boundary of the phantom. (d) Strain generated due to motion of the phantom G- III during the application of current. . . . . . . . . . . . . . . . . . . . . . 37

4.5 (a) Ultrasound A-line as recorded from phantom A-I. (b) Shifting of the front and the rear boundaries of the phantom A-I during the slow time(black lines represent application of voltage across the phantom). (c) Shifting of the whole phantom A-I from the front to the rear boundary of the phantom. (d) Strain generated due to motion of the phantom A-I during the application of current. . . . . . . . . . . . . . . . . . . . . . . . . 38

4.6 (a) Ultrasound A-line as recorded from phantom A-II. (b) Shifting of the front and the rear boundaries of the phantom A-II during the slow time(black lines represent application of voltage across the phantom). (c) Shifting of the whole phantom A-II from the front to the rear boundary of the phantom. (d) Strain generated due to motion of the phantom A-II during the application of current. . . . . . . . . . . . . . . . . . . . . . . . 39

4.7 Fast-time shifting trend changes of a gel $6 \%$ sample occurring during slow time instances 40

4.8 Fast-time shifting trend changes of a gel 3\% sample occurring during slow time instances 40

4.9 Fast-time shifting trend changes of a gel 9\% sample occurring during slow time instances 40

4.10 Fast-time shifting trend changes of an agar 1\% sample occurring during slow time instances 40

4.11 Slow time shifting variations in individual windows of a gel $6 \%$ sample . . . . . . . . . . . 42

4.12 Slow time shifting variations in individual windows of a gel $3 \%$ sample . . . . . . . . . . . 42

4.13 Slow time shifting variations in individual windows of a gel $9 \%$ sample . . . . . . . . . . . 42

4.14 Slow time shifting variations in individual windows of an agar $1 \%$ sample . . . . . . . . . 42

4.15 Amplitude changes near the front boundary of a gel $3 \%$ sample . . . . . . . . . . . . . . . 43

4.16 Amplitude changes near the front boundary of a gel $6 \%$ sample . . . . . . . . . . . . . . . 43

4.17 Amplitude changes near the front boundary of a gel $9 \%$ sample . . . . . . . . . . . . . . . 43

4.18 Amplitude changes near the front boundary of a gel $12 \%$ sample . . . . . . . . . . . . . . 43

4.19 Amplitude changes near the front boundary of an agar $1 \%$ sample . . . . . . . . . . . . . 44

4.20 Amplitude changes near the front boundary of an agar $1 \%$ sample . . . . . . . . . . . . . 44 


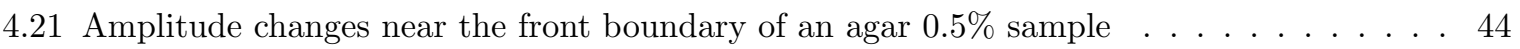

4.22 Amplitude changes within different windows of a Gel $6 \%$ sample $\ldots . . . . .45$

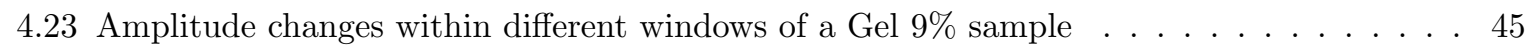

4.24 The shifting fluctuations in a 3\% gelatin sample during the application of electric field. . . 46

4.25 Frequency spectrum of the changes occurring during the application of electric field to a

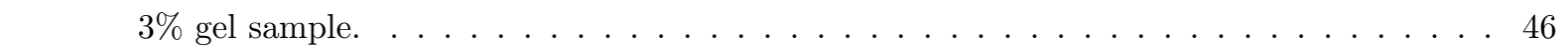

4.26 Linear regression performed on the amplitude of fft with respect to applied voltage of a

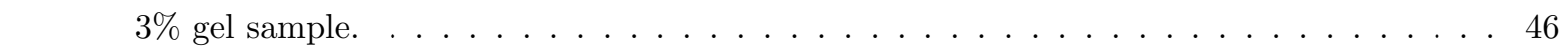

4.27 Frequency spectrum of the changes occurring during the application of electric field to a

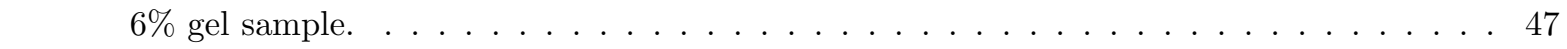

4.28 Linear regression performed on the amplitude of fft with respect to applied voltage $6 \%$

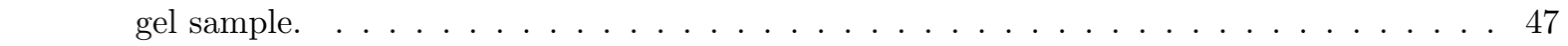

4.29 Frequency spectrum of the changes occurring during the application of electric field to a

$9 \%$ gel sample. . . . . . . . . . . . . . . . . . . . . . . 47

4.30 Linear regression performed on the amplitude of fft with respect to applied voltage $9 \%$

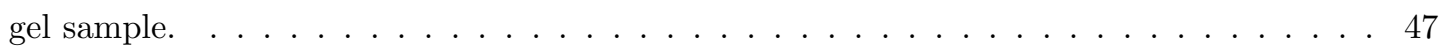

4.31 Slow-time shifting trends for various $3 \%$ gelatin samples $\ldots \ldots \ldots \ldots$. . . . . . . . 48

4.32 Shifting rate comparisons of $3 \%$ gelatin samples to the applied voltage. . . . . . . . . . 48

4.33 Slow-time shifting trends for various $6 \%$ gelatin samples $\ldots \ldots \ldots$. . . . . . . . . 48

4.34 Shifting rate comparisons of $6 \%$ gelatin samples to the applied voltage. . . . . . . . . . 48

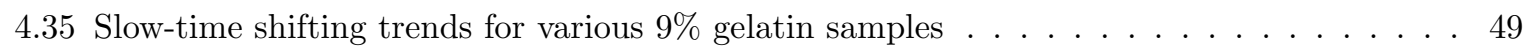

4.36 Shifting rate comparisons of $9 \%$ gelatin samples to the applied voltage. . . . . . . . . . 49

4.37 Variability in $6 \%$ gel experiments $\ldots \ldots \ldots \ldots \ldots \ldots$

4.38 Variability in $1 \%$ agar experiments $\ldots \ldots \ldots \ldots \ldots \ldots$

4.39 Comparision of strain profiles generated for different strengths of gelatin . . . . . . . . 51

4.40 Comparision of strain generated for different strengths of agar . . . . . . . . . . . . 51

4.41 Slow time strain comparison for gelatin phantoms with varying gelatin concentrations at

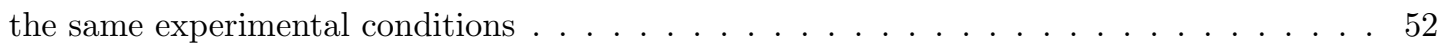

4.42 The dependence of the rate of change of strain on the concentration of gelatin. . . . . . 52

4.43 Slow time strain comparison for gelatin phantoms with varying agar concentrations at the same experimental conditions $\ldots \ldots \ldots \ldots \ldots \ldots 2$

4.44 The dependence of the rate of change of strain on the concentration of agar. . . . . . . . 52

4.45 Effect of increasing salt concentration in $6 \%$ gelatin phantoms $\ldots \ldots \ldots$. . . . . . 53

4.46 Effect of increasing salt concentration in $9 \%$ gelatin phantoms $\ldots \ldots \ldots . . . . . .553$

4.47 Porcine cardiac tissue - Sample I: (a) B-Mode image overlaid with an RGB image generated by the magnitude of the Localised Time Delay Estimates(TDE) before the application of $\vec{E}$ field . (b) B-Mode image overlaid with an RGB image generated by the magnitude of the Localised Amplitude Changes(LAC) before the application of $\vec{E}$ field. (c) TDE overlay after the application of $\vec{E}$ field. (d) LAC overlay after the application of $\vec{E}$ field. (e) B-Mode image of the sample under examination. . . . . . . . . . . . . 55 
4.48 Porcine layered tissue(Muscle and Fat) : (a) B-Mode image overlaid with an RGB image generated by the magnitude of the Localised Time Delay Estimates(TDE) before the application of $\vec{E}$ field . (b) B-Mode image overlaid with an RGB image generated by the magnitude of the Localised Amplitude Changes(LAC) before the application of $\vec{E}$ field. (c) TDE overlay after the application of $\vec{E}$ field. (d) LAC overlay after the application of $\vec{E}$ field. (e) B-Mode image of the sample under examination. . . . . . . . . . . . . . 57

4.49 Porcine cardiac tissue - Sample II : (a) B-Mode image overlaid with an RGB image generated by the magnitude of the Localised Time Delay Estimates(TDE) before the application of $\vec{E}$ field . (b) B-Mode image overlaid with an RGB image generated by the magnitude of the Localised Amplitude Changes(LAC) before the application of $\vec{E}$ field.

(c) TDE overlay after the application of $\vec{E}$ field. (d) LAC overlay after the application of $\vec{E}$ field. (e) B-Mode image of the sample under examination. . . . . . . . . . . . . 58

4.50 Porcine cardiac tissue - Sample III : (a) B-Mode image overlaid with an RGB image generated by the magnitude of the Localised Time Delay Estimates(TDE) before the application of $\vec{E}$ field . (b) B-Mode image overlaid with an RGB image generated by the magnitude of the Localised Amplitude Changes(LAC) before the application of $\vec{E}$ field. (c) TDE overlay after the application of $\vec{E}$ field. (d) LAC overlay after the application of $\vec{E}$ field. (e) B-Mode image of the sample under examination. . . . . . . . . . . . . . . 59

4.51 Gelatin(6\%) phantom(1\% salt) with graphite(5\%) Phantom-I : (a) B-Mode image overlaid with an RGB image generated by the magnitude of the Localised Time Delay Estimates(TDE) before the application of $\vec{E}$ field . (b) B-Mode image overlaid with an RGB image generated by the magnitude of the Localised Amplitude Changes(LAC) before the application of $\vec{E}$ field. (c) TDE overlay after the application of $\vec{E}$ field. (d) LAC overlay after the application of $\vec{E}$ field. (e) B-Mode image of the sample under examination. $\quad$. . 60

4.52 Gelatin(6\%) phantom(1\% salt) with graphite(5\%) Phantom-II : (a) B-Mode image overlaid with an RGB image generated by the magnitude of the Localised Time Delay Estimates(TDE) before the application of $\vec{E}$ field . (b) B-Mode image overlaid with an RGB image generated by the magnitude of the Localised Amplitude Changes(LAC) before the application of $\vec{E}$ field. (c) TDE overlay after the application of $\vec{E}$ field. (d) LAC overlay after the application of $\vec{E}$ field. (e) B-Mode image of the sample under examination. $\quad$. . 61 
Chapter 1

\section{Introduction}




\section{$1.1 \quad$ Introduction}

\subsubsection{Brief History}

\section{Diagnostic Ultrasound}

Diagnostic Ultrasound can be traced back to the World War II era. After the practical application of sonar and radar, physicists as wells as medical practitioners ideated the use of Ultrasound in diagnostic medicene. During the 1940s, the commercial availability of a compact instrument(supersonic reflectoscope) to detect defects in metals blossomed into the idea of medical diagnostics [1]. K.T. Dussik [2] published one of the first papers which suggested that ultrasound can be used for diagnostic purposes. The reflectoscope was applied to the human body in Japan, U.S. and Sweden during this decade [1]. These were the founding stones for ultrasound as a medical imaging modality.

Ultrasonography is a very flexible and versatile imaging modality, but it suffers from low resolution and contrast. Following the development of realtime scanning using ultrasound, 3D and 4D imaging also originated. Researchers started finding techniques to improve ultrasound as a medical imaging modaility. Contrast agents were the first to be developed. Microbubbles [3, 4, being a good contrast agents, were hard to localise and thus limiting their capabilities.

The last few decades were concentrated towards the development of hybrid imaging modalities. Microwave induced acoustic imaging [5], Photo-acoustic imaging [6], Electroacoustic Spectrography [7], Magnetoacoustic tomography with magnetic induction [8] are examples of a new breed of hybrid imaging techniques.

\section{Electric fields and biological tissues/cells}

Electrophysiology and bioelectricity of biological organisms have been studied since the 18th century. Luigi Galvani and Alessandro Volta were the first pioneers to study electrophysiology [9]. By the end of 18 th century and the start of 19 th, definitive evidence for animal electricity was discovered [10]. Electrocardiograph(ECG) signal recording was introduced in 1887 and 1908. Fricke, Cole and curtis were the first researchers who laid the foundations for understanding the cellular/tissue response to electric fields [1]. Schwan followed them with his own discovery of $\alpha$ dispersion to characterize the electrical properties of biomaterials [12]. Electrosurgery started from the 1930s followed by Electroencephalography(EEG) in 1940s and pacemakers, defibrillators in 1960s [13]. Concepts learned over the years have been utilised to develop various diagnostic and therapeutic techniques. Some such examples are displayed in table 1.1 .

\subsubsection{Motivation}

Diagnostic ultrasound is a very well established medical imaging technique. It is non-invasive, versatile and relatively cheap. Efforts have been put together to improve the quality of medical imaging. Probing the functional states as well as the structure of biological tissues is of keen interest to researchers. 
Table 1.1: Applications of electric fields to biological tissues/cells [12

Therapy

UHF-Therapy

Microwave therapy

Wound healing

Laboratory

Coulter Counter

Electronic hematocrit

Diagnostic

Electrocardiography

Impedance Imaging

Electrodiagnosis, Myography

Detection of physiological events by impedance measurements.

Tissue engineering

Ultrasound imaging relies on the mechanical properties of tissues [14. B-mode ultrasound imaging generates grayscale images of the underlying tissues which are characteristics to their backscattering coefficient. The spatial resoultion of the commercial ultrasound scanners is approximately a few hundred microns to a few millimeters. Spatial resolution is the ability to distinguish two closely-spaced objects. However, they suffer from low soft tissue contrast. Contrast is the ability to differentiate different types of tissues. The resolution can be improved by increasing the frequency of ultrasound with a lower penetration depth, while improving the soft-tissue contrast is a challenge. Ophir et al developed the elastography technique which generated strain images based on the the local strain estimates within the tissues due to an axial compressive force [15]. This technique improved soft tissue contrast and potentiated the use of elastographic imaging.

Electrodiagnosis generally employs recording of the endogenic electric potentials as well as the response to exogenic potentials. Impedance imaging generates topographic maps of impedevity by injecting electric current/potentials via multiple electrodes [16]. The contrast provided by this technique is usually fair but it suffers from low spatial resolution. Such a technique could be more useful for understanding the functional state of the underlying tissue.

Electrokinetic effects are observed when exogenic electric potentials are applied to colloidal(microscopic dispersion of one substanse in another) or biological media. As the name suggests, it is defined as the motion of charged particles under the effect of an electric field. A lot of studies have been done on such effects since the 19th century [17. The studies performed have been widely on individual particles, cells and other colloidal materials and it has found applications in places such as sorting cells, segregation of particles etc. There have been very few studies on bulk media, especially bulk biological media. It was found to be challenging while observing such effects in bulk media [18. There have been a few studies which have been done on bulk media(cartilage tissue) where the electro-mechanical changes are observed[19, 20, 21].

Recently, experiments performed on biological tissues under the influence of exogenic electric fields have displayed changes in the ultrasound backscatter signals 22. Changes in amplitude as well as arrival 
time of the US signals received from a tissue have been observed while a physiological level electric field is applied to them. The results also show that effects such as piezoelectricity, electrostriction, Joule heating were ruled out as a cause of effect. It was suggested that electrokinetic effects were playing a major role towards the changes in ultrasound signals and these changes were termed as EIMC(electrical-field induced mechanical changes) 22].

The motivation of this research was to further study and quantify such effects in phantoms as well as ex-vivo biological tissues. Using a clinical ultrasound linear array probe, two dimensional maps of such changes can be obtained which might improve soft tissue contrast based on their electrokinetic properties. Changing properties such as stiffness and conductivity of phantoms might help to understand the nature of such changes. Comparing these changes in US signals based on the stiffness, electrical conductivity and applied electric fields would improve the knowledge of such effects and ultimately lead to characterisation of biological tissues based on their electrokinetic properties(electrophoresis, electroosmosis etc.).

\subsubsection{Organisation of chapters}

The first chapter described a brief history and motivation for this research. The second chapter includes an in-depth literature review pertaining to this study. The third chapter describes the materials and methods used for the research and defines the terms used in this study. It will be followed by a presentation of results and observation of the dependence of the EIMC on experimental parameters. The last chapter includes discussion, conclusions and futurework. 
Chapter 2

\section{Literature Review}




\title{
2.1 Tissue-mimicking Phantoms used in ultrasound studies
}

A wide variety of tissue-mimicking phantoms are being used in ultrasound studies. The aim of phantoms is to serve as consistent targets for calibration, ultrasound system testing and training. The most important properties of soft-tissues which are to be mimicked include the compressional speed of sound, characteristic acoustic impedance, attenuation, backscattering coefficient and the non-linearity parameter 23]. For hard tissues, these properties are compressional and shear wave speeds of sound, characteristic acoustic impedance and attenuation [24. Another important advantage of phantoms is that being easily customizable; they can adhere to high specificity. Controlling individual properties of such phantoms is easier and hence aids in better characterization of novel effects. Preparation of phantoms has been studied in detail and there have been many different types of materials being used to prepare such phantoms. A list of some of the gelling agents being used in preparation of phantoms is displayed in table 2.1. These agents have been tabulated from a comprehensive review presented in 24] where they were described in more details. There are many challenges presented when designing

\author{
Agarose-based \\ Gelatin-based \\ Magnesium Silicate-based \\ Oil gel-based \\ Open Cell Foam-based \\ Polyacrylamide Gel-Based \\ Polyurethane \\ Polyvinyl Alcohol-based \\ Tofu \\ Water-based \\ Condensed Milk-based \\ Urethane Rubber \\ Zerdine
}

Table 2.1: Some gelling agents used for preparing ultrasound phantoms [24]

customized tissue-mimicking phantoms for a particular study. Speed of sound and attenuation are highly dependent on temperature. Elastic properties of biological tissues are dependent on many factors such as the age, body location, the state of tissues(in vivo, ex vivo), fiber orientation and loading [23, 25. The choice of phantoms for this study were finalized after reviewing various studies [26, 27, 28]. Two gelling agents were used in this study Agar and Gelatin. Gelatin and agar are widely studied and used for preparing tissue-mimicking phantoms. They were used in this study as it would be easier to control the physical and chemical properties to investigate their effects on EIMC.

\subsubsection{Gelatin}

Gelatin is defined as a protein formed by hydrolysis of collagen extracted from skin, white connective tissue and bones of animals [29]. Collagen is considered to be the principle proteinaceous component of the white connective fibrous tissues. They serve as the chief tensile stress-bearing elements for all 
vertebrates [30. Gelatin belongs to a class of Hydrocolloids (colloids dispersed in water). The major applications of gelatin include usage in preparation of food items, medical usage for preparing capsules etc., coating of photographic films, and preparing tissue-mimicking phantoms [31. Gelatins are classified in accordance to the method of manufacture. Acid treated gelatins are denoted as type-A gelatins whereas alkaline treated gelatins are treated as type-B gelatins [31].

\section{Structure}

All types of gelatins are prepared basically from collagen hence the structure of gelatin bears a strong resemblance to collagen. Tropocollagen, the collagen monomer is a triple helix or rod of about $300 \mathrm{~nm}$ long and 1.5 $\mathrm{nm}$ diameter of a molecular weight of 300,000 [31. In solution, collagen monomers form cross links which increase in tensile strength with age. Commercially available gelatins are very heterogeneous in molecular size because of these stable cross links which affect the manufacturing process. [31]. Figure 2.1 shows a microscopic structure of low and high concentration of gelatin gels. The fishnet structure of gels displays the gelatin filaments of lengths between 20 and $100 \mathrm{~nm}$ for $2 \%$ gels and 10-30 $\mathrm{nm}$ in $10 \%$ gel 32 . The $2 \%$ gelatin gel shows a disordered network with meshes of variable sizes whereas the $10 \%$ gel shows a homogeneous and dense network. At temperatures above $35-40^{\circ} \mathrm{C}$, gelatins in solutions behave as randomly oriented coils. They are disordered and have no structure. On cooling, concentrations higher than $1 \%$ would form a clear gel depending on the quality of gelatin and its $\mathrm{pH}$. Gelatin is a type of physical gel which is formed by hydrogen bonds. This makes them thermo reversible and on warming the gels to $35-40^{\circ} \mathrm{C}$, dissolution occurs. The pyrolidine-rich junctions of gelatin form inter chain hydrogen bonds which forms the basic framework of gelatin gels [31. Dry gelatin has practically an infinite shelf life, if stored under the proper conditions. When preparing a phantom, the water dipoles adsorb to the protein structure of gelatin [33. If additives are included in the phantom, it is possible that a few positively charged ions adsorb to the negatively charged structure of gelatin based on their electronegativity [33. Gelation is the term generally employed to define the mechanism of gel formation. A concentration $c_{o}$ can be defined to be the critical concentration of gelatin above which gelation occurs.

The speed of sound in gelatin phantoms can be controlled with different types of additives (an example would be n-propanol). Some example ranges can be found Physical properties of tissues by Francis A. Duck 34]. Ranges from 1500-1580 m/s and higher have been reported and can be controlled with relative ease. Attenuation coefficients ranging from $0.368-1.453\left(\mathrm{~dB} \mathrm{~cm}^{-1} \mathrm{MHz}^{-b}\right)$ where b ranges from 0.99-1.24 have been reported [34. These values were reported at a temperature of $25 \mathrm{C}$.

\section{Concentration dependence of gelation}

Studies in the past have suggested that the dependence of the equilibrium shear modulus $G_{e}$ (the ratio of sheer stress to sheer strain under equilibrium conditions) on the concentration of gelatin is quite variable 29]. There were some specific ranges of concentrations where it was found that $G_{e} / c^{2}$ or $G^{\prime} / c^{2}$ had a constant value indicating that $G^{\prime}$ (storage modulus-ratio of stress to strain which includes the phase difference between stress and strain for viscoelastic materials) or $G_{e}$ are linear functions of $c^{2}$ 
a

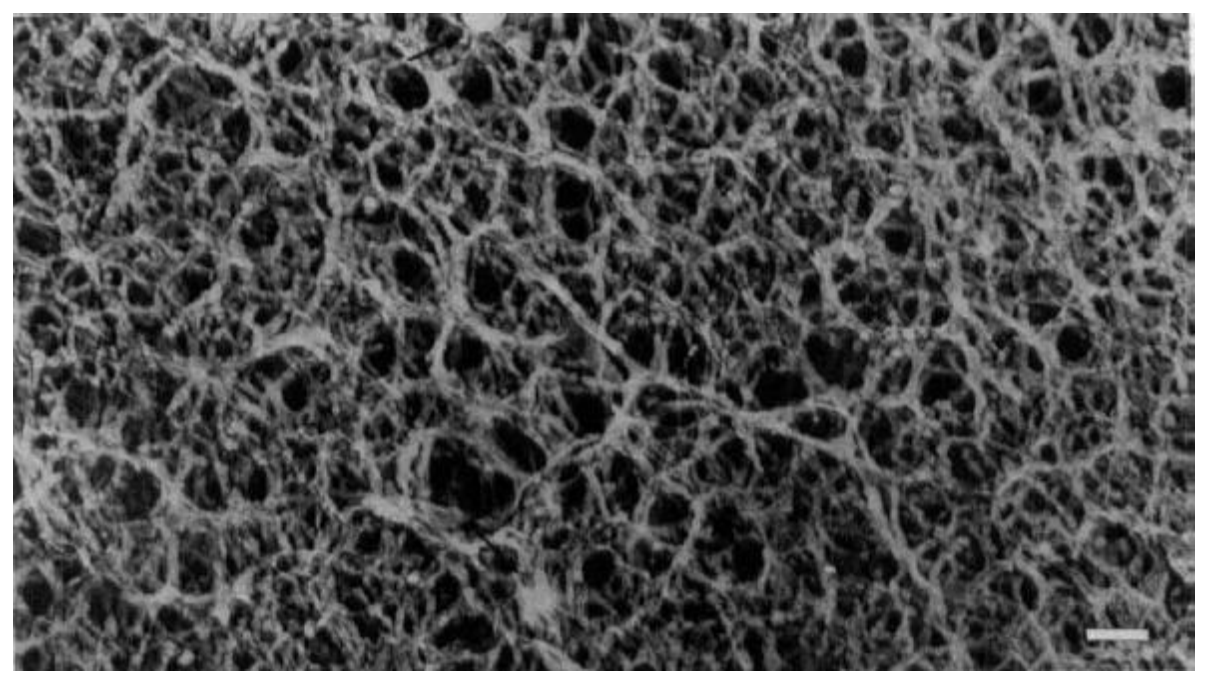

b

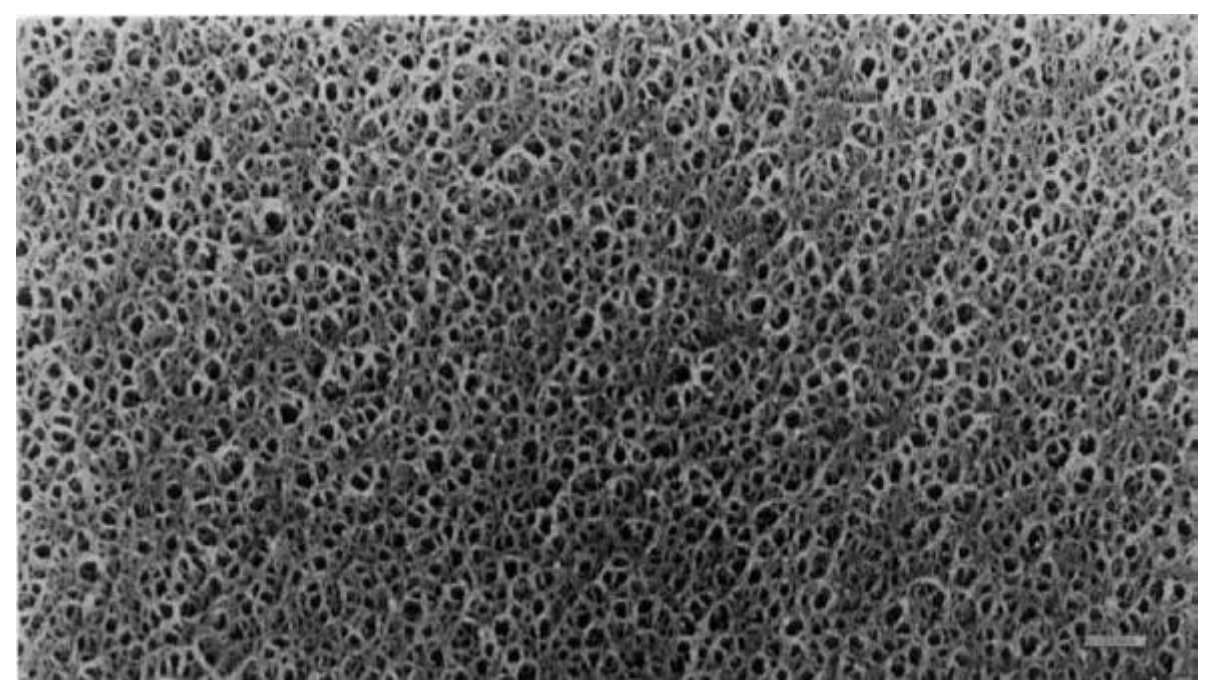

Figure 2.1: Microscopic images of (a) 2\% gelatin gel and (b) 10\% gelatin gel. The white bar on the lower right corner represents $100 \mathrm{~nm}[32$ 
[35, 36, 29, 37. The doctoral thesis of Te Nijenhuis [35] shows that for $2-5 \mathrm{wt} \%$ gelatin solutions, plots of $G^{\prime}$ vs $c^{2}$ were found to be linear for aging times ranging from 0.3 to 100 hours 2.3 . This power law dependence is not completely satisfied for the concentration range of $10-50 \mathrm{wt} \%[29$. Figures $2.2 \& 2.3$ show such dependence.

It has also been shown that when salts are added while forming gels, the properties of the gel might be affected. The value of storage moduli recorded in [38] suggests the softening of gels at high $\mathrm{NaCl}$ concentrations. The effect of adding salt was seen to be similar to the effect of an increase in temperature for gelatin gels.

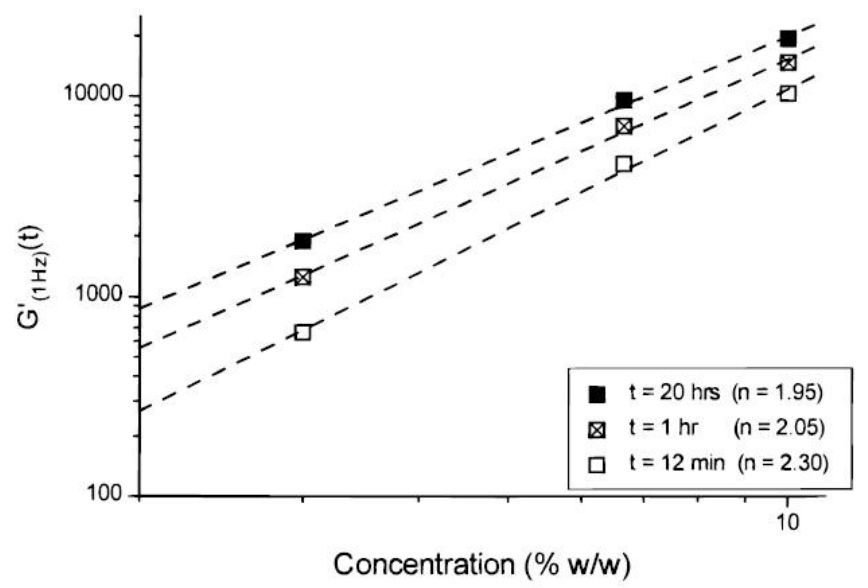

Figure 2.2: Elastic modulus of gelatin as a function of concentration extracted from [36]

\subsubsection{Agar}

Agar is a linear polysaccharide which consists of two major components, agarose and agaropectin[39]. It dissolves in water at temperatures above $90^{\circ} \mathrm{C}$. Agar forms physical gels in which the structure is formed solely by the production of hydrogen bonds within the polymer chains 31. The mechanism of their gelation is very similar to gelatin with a difference in the amount of agar required to form gels. A $2.5 \%$ agarose gel has a pore size of approximately $500 \AA$ [ 40 . Figure 2.4 displays the comparison of gelatin and agar gels. It also defines critical concentrations $c_{o}$ for agar $(0.17 \%)$ and gelatin $(1.2 \%)$. Speed of sound range could be controlled in agar phantoms in a similar fashion as gelatin phantoms. Attenuation coefficients ranging from $0.124-1.62\left(\mathrm{~dB} \mathrm{~cm}^{-1} \mathrm{M} \mathrm{Hz}^{-b}\right)$ where b ranges from 0.94-1.18 have been reported [34]. These values were reported at a temperature of $22 \mathrm{C}$.

\section{Concentration dependence of gelation}

Unlike Gelatin, there is no widely accepted dependence of stiffness on concentration for Agar. Many different regions exist where there can be suggestions that there is a concentration dependence for the 


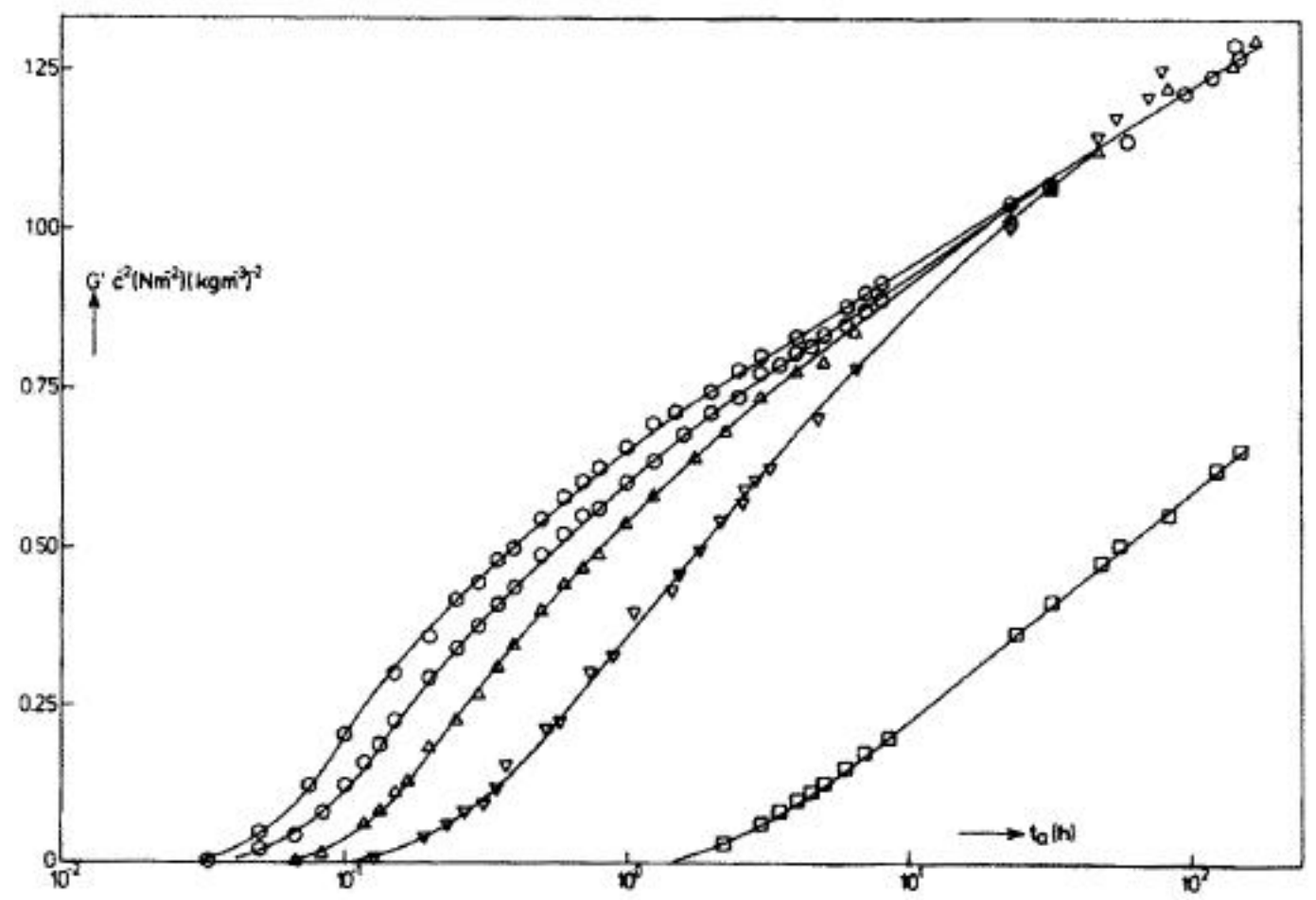

Figure 2.3: A plot displaying the ratio of $G^{\prime} / c^{2}$ vs ageing time in hrs. Gelatin weight $\square-1.00 \% \nabla-$ $1.95 \% \triangle-3.00 \% \bigcirc-4.00 \% \bigcirc-5.00 \%$ extracted from [29] 


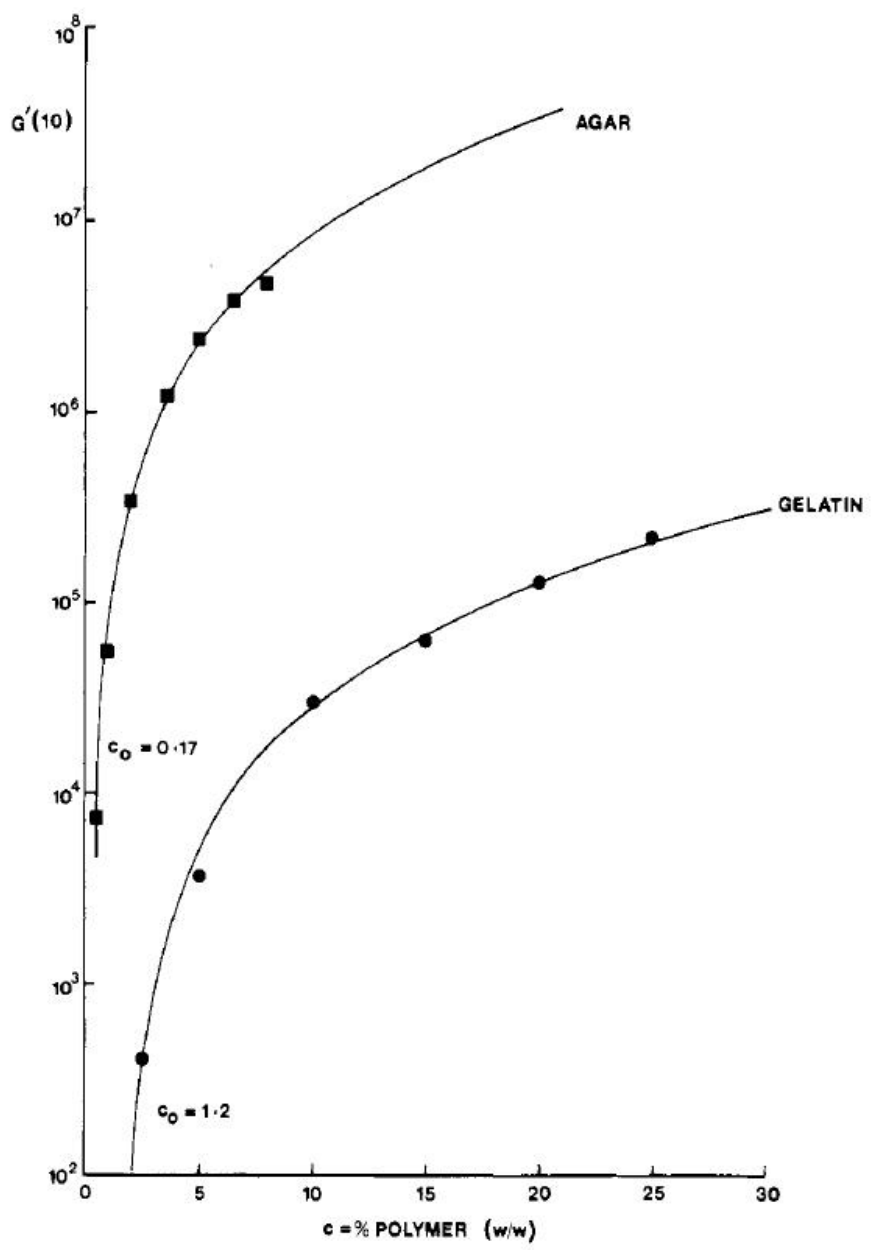

Figure 2.4: The dependence of $G^{\prime}(10 \mathrm{rad} / \mathrm{s})$ on the concentration of gelatin and agar (extracted from [42])

storage modulus $G^{\prime}$. For gelatin, the dependence is found to be on $c^{2}$ where for agar, it is not such a simple dependence. It highly depends on the type of agarose and the dependence has found to be varying from an exponent of 2 to an exponent of 4 [4]. From figure 2.4 it can be seen that to prepare a gel with a higher $G^{\prime}$, substantially higher amount of gelatin is needed when compared to agar.

\subsection{Conductivity of saline solution}

Figure 2.5 show conductivity versus concentration for saline solution. It is seen in the figure that the conductivity depends on frequency and the increase in concentration increases conductivity in a linear sense. The conductivity of gelatin and agar phantoms is expected to be similar to such observed values. 


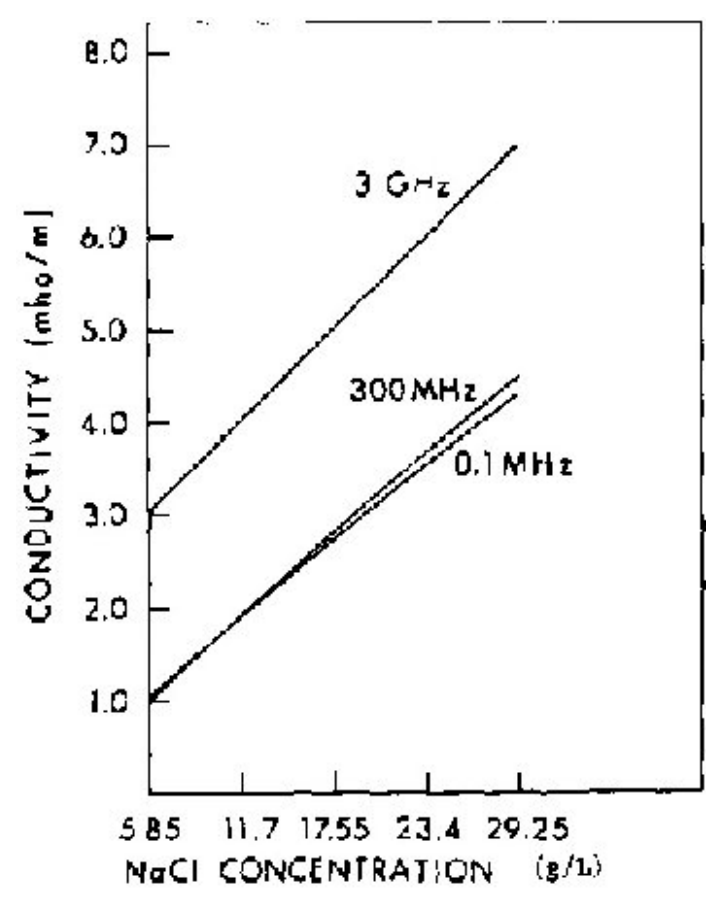

Figure 2.5: Conductivity of various concentrations of saline [43]

\subsection{Concepts of Bio-Electric Fields}

Biological tissues are complex anisotropic electrochemical systems. Electrical fields, when discussed in the context of biological media, can be classified into two types; Exogenic \& Endogenic [44. Exogenic fields are attributed to the application of electric fields using an external source independent of the biological system. Endogenic electric fields are internally generated within the biological media due to the inherent biological activities of the system. The best examples of Endogenic electric fields are the fields aiding in the conduction of neuronal impulses, pacing of heart, transport of ions through the biological ion channels etc. [44]. The study of Endogenic electric fields in itself is a very wide subject and hence will not be pursued in detail as it is out of scope of this thesis.

When external sources are used to apply an electric current/voltage to biological tissues, Exogenic electric fields are generated in response. Such electric fields are applied either for therapeutic or diagnostic purposes (Electrical Impedance measurements [16]). The anisotropy of tissues increases with the increase in frequency. The local distribution of electric fields depends on the chemical and physical nature of the type of tissues. In order to understand the response of tissues, we need to review the concepts of electrical conductors, dielectric properties, electric fields in electrolytic solutions, and effects such as polarisation and interfacial electric double layers and the structure of tissues.

The most common definition of conductor is A material which allows the electrical current to flow through. The opposition caused by a material to the flow of electrical current is termed as resistance $R$ (when dealing with DC electric fields) or impedance $Z$ (when dealing with AC electric fields) [44. The 
magnitude of Electric field (E) is measured in units of $V / m$ and the current density $(\mathrm{J})$ is measured as $A / m^{2}$. The electric field is measured across the length of a concuctor and the current density is measure through the cross section as shown in figure 2.6. Based on these known values, the resistivity of a material can be expressed as

$$
\rho=\frac{E(V / m)}{J\left(A / m^{2}\right)}
$$

The conductivity is defined as the inverse of $\rho$ and its units are $m h o m^{-1}$ or $\operatorname{Siemens}(S) m^{-1}$. Another

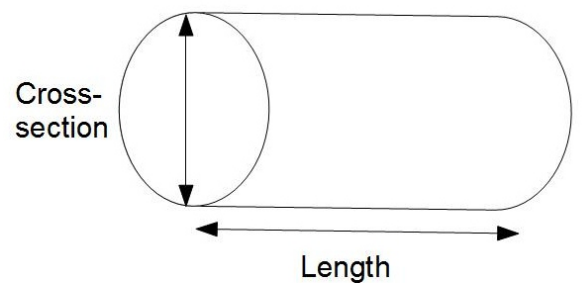

Figure 2.6: Schematic representation of a cylindrical conductor

important parameter when discussing about the electric field induction is Permittivity $(\epsilon)$. It is defined as the ability of a material to hold electrical charge. In case of tissues, a complex permittivity is defined which incorporates the phase difference representing the causal nature between the external electric field and the response it generates.

For metallic conductors, the flow of electric current is actuated by the movement of electrons. Electrolytic medium can also act as a conductor as long as it has free ions to conduct the electricity. An electrolyte can be considered as a substance with ionic dc conductivity. The nature of the ions present in the electrolyte as well as the method of applying electric field plays an important role in the flow of current through the electrolyte [44. Temperature rise in tissue volume due to the flow of electric current is given as

$$
\Delta T=\frac{J^{2} t}{\sigma c d}
$$

where $\mathrm{J}$ is the current density, $t$ is the time, $\sigma$ is the conductivity, $c$ is the specific heat capacity and $d$ is the density. 44

\subsection{Exogenic electric fields in tissues}

An organised tissue structure exhibits a different response to an application of AC or DC exogenic fields. As shown in the schematic (Fig. 2.7), a low frequency/DC current passes only through the extracellular space whereas the high frequency current passes through the intracellular as well as the extracellular space 45, 46]. This is due to the fact that the membrane acts as a capacitor blocking the low frequency/DC current. The flow of current is also affected by the macroscopic and the microscopic anisotropies. Figure $2.8-2.9$ show such a schematic representation describing the macroscopic and the microscopic anisotropies. A DC field is affected by both of these anisotropies whereas an AC field is not affected by the microscopic anisotropies. To describe tissues, many electrical models have 


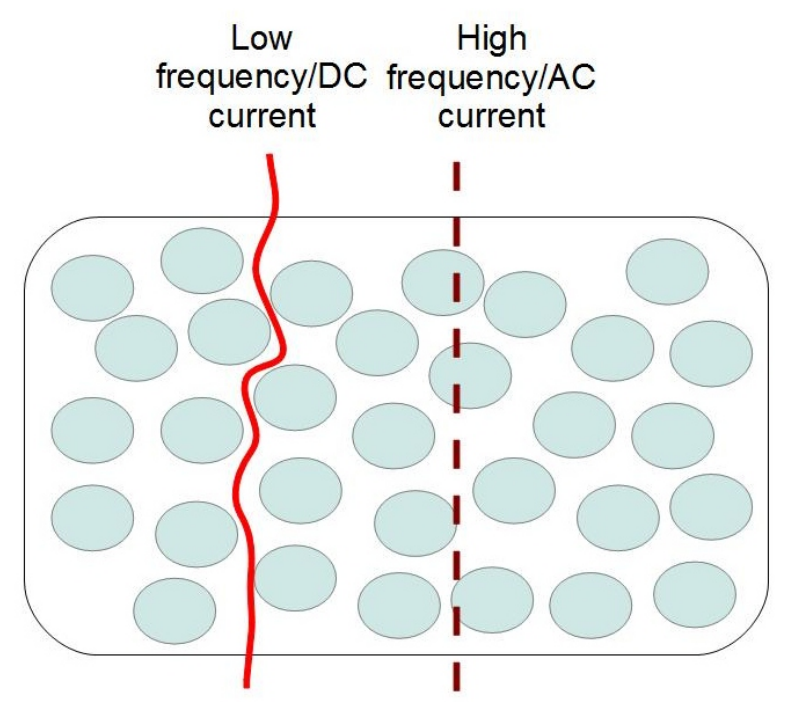

Figure 2.7: Flow of high frequency vs low frequency current through tissues (a schematic). The arrangement represents cell (blue ovals) and the extracellular matrix(white space around the ovals).

been devised 47, 48, 49, 50, 51. The most basic model to describe the electrical nature of tissues is described in figure 2.10 The cell membrane acts as a capacitor which separates the extracellular and the intracellular components. Skin is an important tissue when it comes to dealing with electrical characteristics for the body. Skin acts as the primary interface between the source applicator and other internal organs. When designing models, skin plays an important role. The resistivity of skin is very high when compared to the other tissues of the body [52, 34]. Dry skin has DC conductivity values from $0.00002-0.0002 S / m$ [52]. The maximum conductivity of skin was found in its underlying layers where the conductivity values could be around $0.227 \mathrm{~S} / \mathrm{m}[52$. It shows that skin is very inhomogeneous and hence their electrical properties vary in such a fashion. Skeletal muscle tissue is strongly anisotropic with low-frequency conductance ratio of about 1:8 between the transversal (perpendicular to the muscle fibres) and longitudinal directions (parallel to muschle fibres) [44. The values of DC conductivity in longitudinal direction range from $0.3-0.8 \mathrm{~S} / \mathrm{m}$ whereas the values in the transversal direction range from $0.04-0.14 S / m$ [52]. The DC conductivity of fat tissues lies between $0.02-0.04 S / m$. Fat tissues are considered to be more homogeneous than skin or muscle tissues.

\subsection{Applying AC and DC electric fields to biological tissues}

Human body is composed majorly of water, organic and inorganic materials. The inorganic materials are mostly in the form of ions residing the the extracellular as well as the intracellular fluids of the body [53. The presence of these makes the human body conductive and the electrical properties of the human body can serve as a contrast media for imaging. Electrical impedance tomography is such an imaging modality which generates images based on the impedance of biological tissues. The application of 

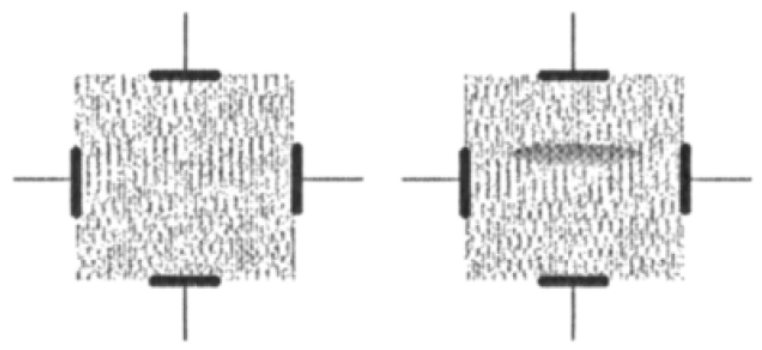

Figure 2.8: A schematic of macroscopic anisotropies, extracted from 44. Layers such as fat, muscles, and bones form anisotropies on a macro-scale.

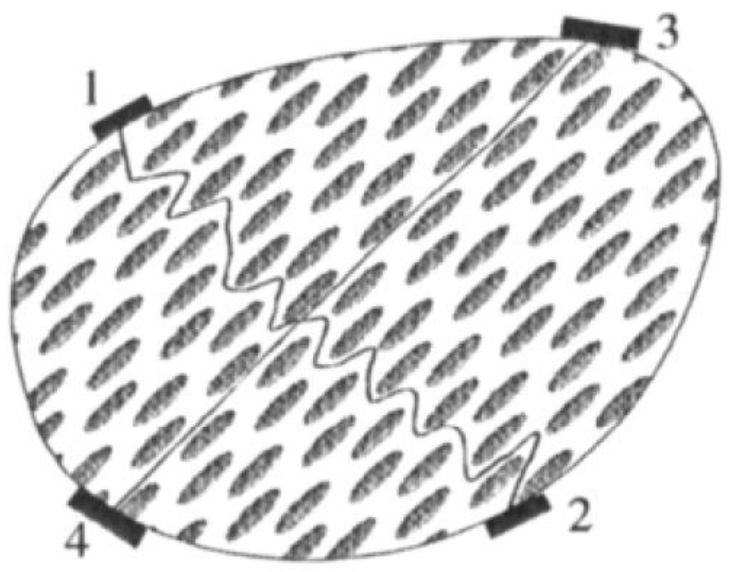

Figure 2.9: A schematic of microscopic anisotropies, extracted from [4]. Microscopic arrangement of cells is responsible for the microscopic anisotropies.

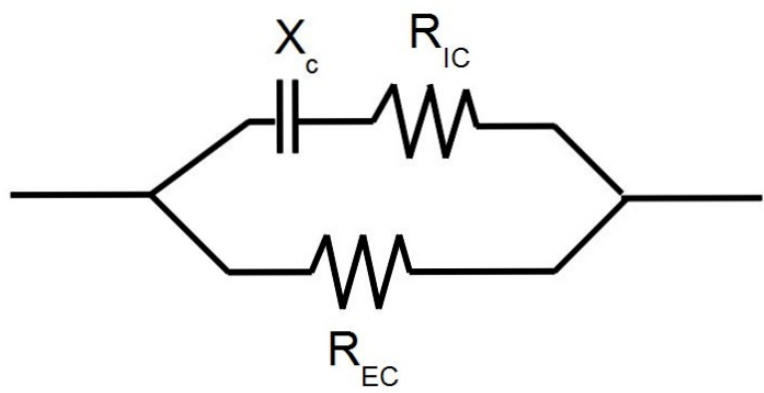

Figure 2.10: A simple circuit describing the capacitance of the cell membrane $\left(X_{c}\right)$, Resistance of the intracellular components $\left(R_{I C}\right)$, Resistance of extracellular components $\left(R_{E C}\right)$ 
electric fields to tissues elicits various responses based on the type and nature of the electric field applied. Table 2.2 provides a short review of the effects of electric fields on biological media. Electroporation is

\section{Effects of electric fields}

Electroporation [54]

Electrodeformation/Cell-shape changes [55, 56 ]

Orientation/migration depending on the direction of applied field [57, 58,

Galvanotaxis [59, 60, 61

Iontophoresis 62, 63, 64]

Electrokinetic Phenomena[65, 44]

Electrophoresis

Electro-osmosis

Streaming current and Potential

Electroacoustics

Sedimentation potential

Table 2.2: A collection of the response of tissues/cells to electric fields

generally defined as the formation of pores in the cell membrane in response to the application of high frequency electric fields. This effect has been used in drug/gene delivery to specific cells. As the name suggests, Electrodeformation or cell shape changes is defined as the changes imparted to the morphology of a cell by electric fields. Both high frequency and low frequency electric fields can be responsible for these changes. Galvanotaxis is the movement of cells in accordance to the applied electric fields. Iontophoresis is termed as driving particles across the cell membrane based on an applied electrical gradient. Electrokinetic phenomena is a term collectively used for describing the kinetic effects induced due electric fields and vice versa.

\subsection{Ultra-low frequency/DC and the importance of intersti- tium}

The response of tissues to the application of ultra-low frequency/DC electric fields is majorly attributed to the extracellular space 44, 45] as the current flows through it. The extracellular space is divided into the vascular space and the interstitium. The interstitium mostly comprises of the interstitial fluid and the extracellular matrix to which the cells fixate [53. Figure 2.11 shows the structure of interstitium. The two major solid structure present in the interstitium are the collagen fiber bundles and the proteoglycan filaments (polysaccharide network) [53, 66] with other structures such as non-collagenous glycoproteins 66]. The collagen fibers are responsible for the tensile strength as they are long and extremely strong. The combination of proteoglycan filaments and water make a gel like structure surrounding the cells. The flow of fluid is restricted due to such a gel-like structure and hence the fluid diffuses through this gel [53. The proteoglycan molecule is composed of a single protein core to which glycosaminoglycan(GAG) chains are attached. The dimeric units of GAGs are chondritin sulfate(CS) and keratan sulfate(KS) 67]. Under normal physiological conditions, $\mathrm{CS}$ has two negatively charged $\operatorname{groups}\left(\mathrm{SO}_{3}^{-} \& \mathrm{COO}^{-}\right)$and $\mathrm{KS}$ has one negatively charged $\operatorname{group}\left(\mathrm{SO}_{3}^{-}\right)$. Many such chains are attached together to form a single 


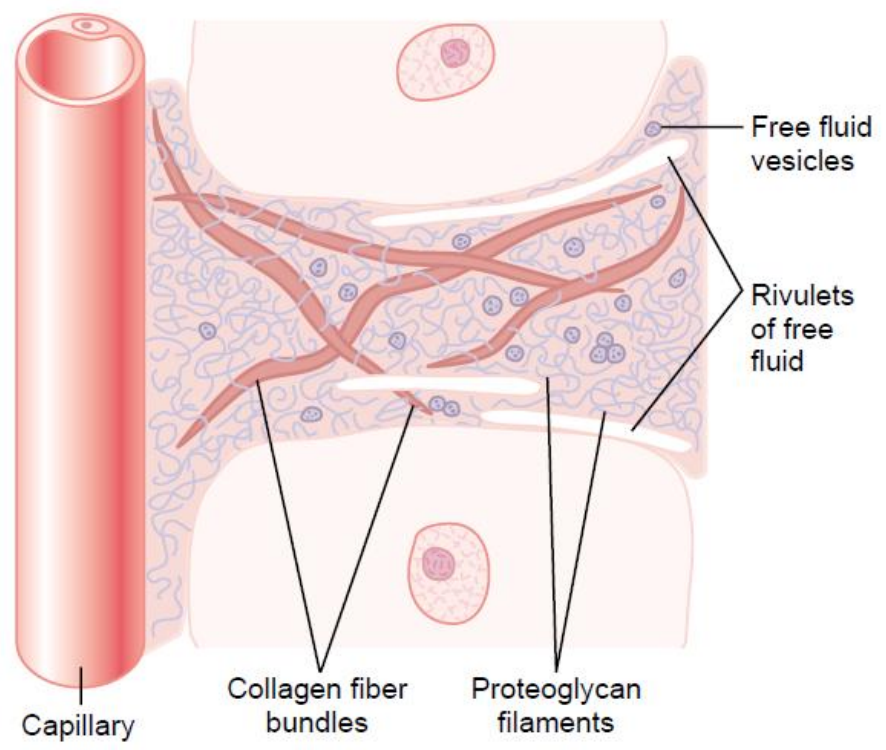

Figure 2.11: A pictorial depiction of the interstitium $[53$

hyaluron molecule which tends to be negatively charged due to such groups present. Since these charges are connected to the proteoglycans which are a part of the extracellular matrix, these charges are immobile and hence they are known as fixed charges. Their density per volume of the interstitial water is termed to be the fixed charge density(FCD). Apart from solid structures, there are ions present in the interstitial fluid. These ions have a different concentrations in the interstitial fluid and the intracellular fluid. Ions such as $\mathrm{Na}^{+}, \mathrm{K}^{+}, \mathrm{Ca}^{++}, \mathrm{Mg}^{+}, \mathrm{Cl}^{-}, \mathrm{HCO}_{3}^{-}, \mathrm{HPO}_{4}^{-}, \mathrm{SO}_{4}$ are present in the interstitial fluid. With the exception of $\mathrm{Mg}^{+}, \mathrm{K}^{+}, \mathrm{HPO}_{4}^{-} \& \mathrm{SO}_{4}$, all the other ions have a high concentration in the interstitial fluid(when compared to the intracellular fluid) [53. The cations present in the interstitial fluid are attracted towards the negatively charged proteoglycans which in turn preserves the electroneutrality on a macro scale 67. These interactions at the molecular level are primarily responsible for mechanoelectrochemical phenomena like electro-osmosis, streaming current, sedimentation potential (described later on in the chapter) on a macroscopic scale.

\subsection{Electrokinetic phenomena}

According to the International Union of Pure and Applied Chemistry (IUPAC) technical report 65], "Electrokinetic(EK) phenomena can be loosely defined as all those phenomena involving tangential fluid motion adjacent to a charged surface." EKs has a close connection with the fields of electrical double layers and electro-static surface forces. There are many different types of EK effects as defined in table 2.2

The motion of charged colloidal particles or polyelectrolytes immersed in a liquid due to the application of 
an external electric field is termed as Electrophoresis. Electro-osmosis is the opposite of electrophoresis, it is defined as the movement of liquid through immobilized particles or a porous structure. Streaming Potential is defined as the potential caused by the motion of a liquid under a pressure gradient through a capillary or a porous structure. When colloidal particles sediment under gravitational force, an electric field can be recorded via electrodes immersed in the solution at a known distance apart. This electric field is known as the sedimentation potential. All these effects involve the motion of either the colloidal particles or the fluid. Dielectrophoresis is the movement of non-charged particles in an electric field. Dipoles have a net charge of zero but they do observe a rotational force in a homogenous electric field. In an inhomogeneous electric field, there can also be a translational force acting on a dipole [44.

To determine the electrical state of a charged surface, it is essential to know the spatial distribution of ions around it. This is envisioned by the concept of electrical double layers. Figure 2.12 describes a simplest electrical double layer which can be formed near a charged surface. The positive surface charges are bound to the colloid particle and is considered to be the first layer. The second layer is distributed in solution where the colloid particle is immersed. The counter-ions (negative in this case) are highly concentrated near the surface of the particle whereas the co-ions (positive in this case) are scarcely distributed in the immersed liquid.

This simple structure is not appropriate to describe the complex response occurring at such interfaces.

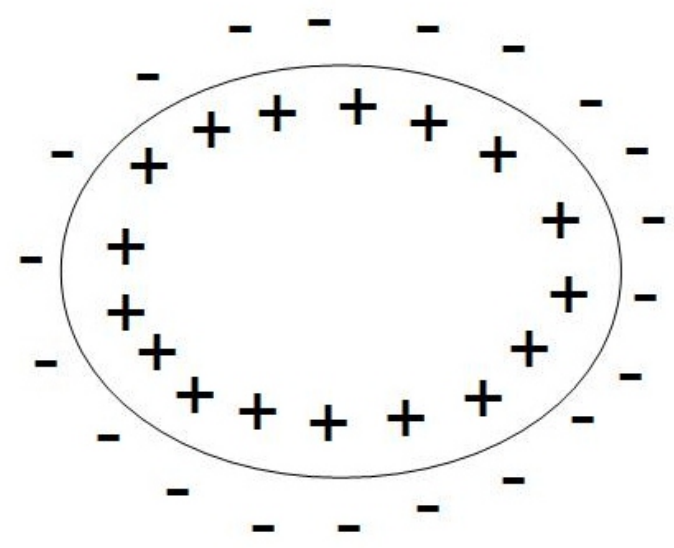

Figure 2.12: Schematic describing a primitive Electrical double layer

Hence, the electrical double layers are further divided and enhanced to theoretically explain the phenomenon. The uncharged region between the surface and the collection of counter ions is known as the stern layer and the ions present beyond the collection of counter ions form the diffuse of Gouy layer. To explain the effect further, the stern layer is divided into an inner Helmholtz layer (IHL) which is bounded by the surface and the inner Helmholtz plane (IHP). Another outer Helmholtz layer (OHL), located between the IHP and the outer Helmholtz plane(OHP) also forms a part of the stern layer as displayed in figure 2.1365$]$.

$\sigma^{0}, \sigma^{i}, \sigma^{d}, \sigma^{e k}$ are the fixed surface charge density, the IHP charge density, the diffuse-layer and the 


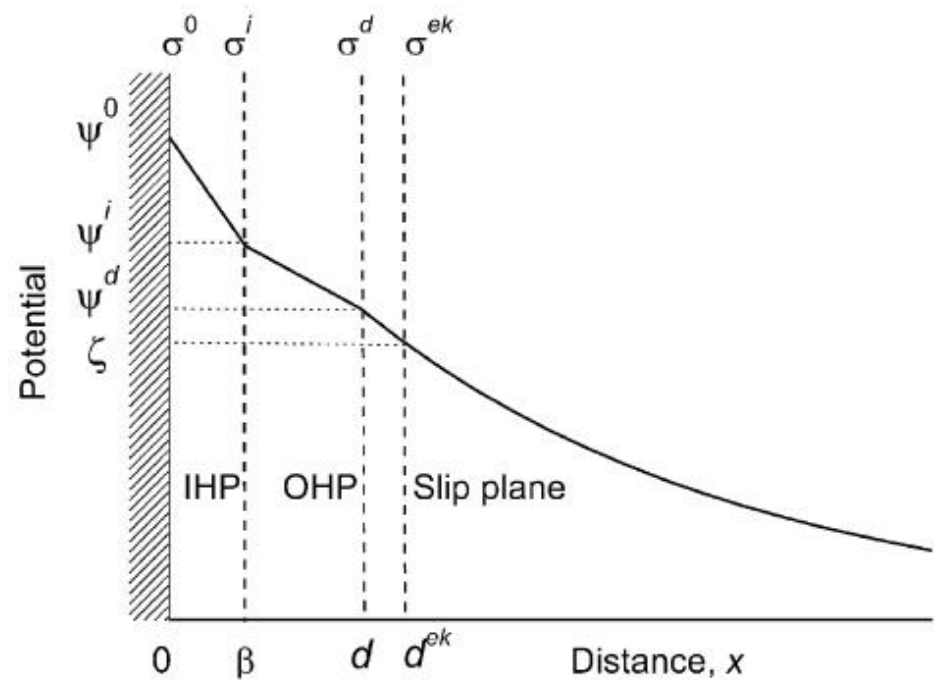

Figure 2.13: A detailed schematic of an electrical double layer formed near the surface of an electrolyte 65

electrokinetic charge density respectively. The potential at every layer are defined by $\Psi^{0}, \Psi^{i}, \Psi^{d}, \zeta$ respectively. The $\zeta$ potential is an important term as it defines the potential at the slip plane which is hydrodynamically active unlike the other layers [65. The distances at which these layers form depend on the medium and the suspended particles [65].

The electroosmotic velocity is given by the Smoluchowski equation [65] which is

$$
\nu_{e o}=-\frac{\epsilon_{r s} \epsilon_{0} \zeta}{\eta} E
$$

where $\epsilon_{r s}$ is the relative permittivity of the electrolyte solution, $\epsilon_{0}$ is the electric permittivity of vacuum and $\eta$ is the dynamic viscosity of the liquid.

Similarly, the electrophoretic velocity is given by

$$
\nu_{e}=\frac{\epsilon_{r s} \epsilon_{0} \zeta}{\eta} E
$$

where the negative sign has changed to positive as it defines the velocity of the particle with respect to the medium at rest.

\subsection{Recent progress in the area}

Electrokinetic phenomena has been studied extensively in the field of colloid chemistry. Many widely accepted theoretical models and solutions have been presented in this domain. All such studies dealt on a microscopic scale and either on single colloids 68, 69, 70] or a few natural fibers [71, 72, 73]. Studies on 
bulk biological media are complex and very few studies have tried to study and characterize such effects in bulk media.

There have been some studies on the response of certain tissues to electrical and photo-thermal stimulation[21]. These studies indicated that there were electrokinetic effects observed using optical coherence tomography 20 , 19]. In [19], the authors conclude that since the EK effects are directly related to the proteoglycan density of the articular tissue, using OCT combined with the application of electric fields would provide a tool to evaluate the viability of the articular tissues. In [21, the authors used a phase sensitive low coherence reflectometer to measure path lengths inside an atherosclerotic artery during the application of electric field. While recording the displacements in the articular tissue in response to the application of electric fields, the authors found that the maximum displacements occurred at the zero crossings of the electrical cycles. They also found that at lower stimulation frequencies, the displacements were larger.

Recent experiments performed in 22] have shown the electro-mechanical changes induced by electric fields in tissues can be detected using ultrasound. These changes indicate tissue motion and its respective strain. Effects such as electrostriction, piezoelectricity, joule heating have been ruled out to be a cause of such changes. The studies were performed using a single element transducer and some results showed a difference in changes respective to the type of the tissues (muscle or fat).

\subsection{Hypothesis and specific objectives}

Hypothesis: Electric field-induced mechanical changes in biological tissues and ultrasound phantoms can depend on the electric field strength, the sample stiffness and conductivity. The localised maps of such changes due to the electrokinetic effects can be formed using B-mode ultrasound imaging.

\section{Specific objectives}

- Study the electrokinetic effects in tissue-mimicking Phantoms.

- Observe the dependence of EK effects on the electrical field strengths, concentration of the gelling agents and conductivity of such Phantoms.

- Generate localised EK maps from B-mode US images based on changes in RF signals during the application of exogenic electric fields in tissues and phantoms. 
Chapter 3

Materials and Methods 


\subsection{Important terms used in this chapter}

Slow time(s) - It represents the instant when ultrasound transducer was triggered and also the time line of the application of voltage source to the sample.

Fast time(s) - The time corresponding to the triggering of the transducer and acquisition of reflected echoes.

Shifting fluctuations(ns) - The changes observed in shifting in the windows induced by the application of electric field.

Amplitude changes $(V)$ - The changes observed in amplitude of windows induced by the application of electric field.

Shifting trends(ns) - The trends observed in time shifting during the application of electric fields.

\subsection{Preparation of Phantoms}

The method of preparing phantoms was developed after reviewing studies [26, 27, 28].

\section{Gelatin Phantoms}

In order to prepare Gelatin Phantoms, de-ionized water was utilized. Type-A gelatin powder(3g12g)[G2500-Sigma Aldrich], extracted from porcine skin, was added to the de-ionized water $(100 \mathrm{ml})$ and was constantly stirred. The mixture was then heated upto $70^{\circ} \mathrm{C}$. Application of heat was turned off after a clear solution was seen indicating the dissolution of gelatin in the water. Depending on the type of phantom, graphite was added $(5 \mathrm{~g}-7 \mathrm{~g})$ and the mixture was cooled . After mixing, the phantom was transferred to its mould and allowed to cure at room temperature $22^{\circ} \mathrm{C}$ for approximately $24 \mathrm{~h}$. Care was taken to avoid fomation of bubbles while mixing. The prepared phantom was then submerged in vegetable oil and allowed to thermally equilibriate for 1-2h. Experiments were then performed on the phantoms.

\section{Agar Phantoms}

A similar approach was followed for agar[LP0011-Oxoid Limited] phantoms. The temperature was increased to approximately $90^{\circ} \mathrm{C}$ and the solution was heated until a clear solution was obtained in case of agar.

\subsection{Experiments including single element transducers}

The experimental setup for analyzing the US echo signal changes comprised of a tank filled with a fluid (water or vegetable oil as depicted in figure 3.1 ). The samples $(6 \mathrm{~cm} \times 3 \mathrm{~cm} \times 2 \mathrm{~cm}$ ) to be analysed were placed on a plastic base. In order to apply electric fields to the samples, copper electrodes were used. 


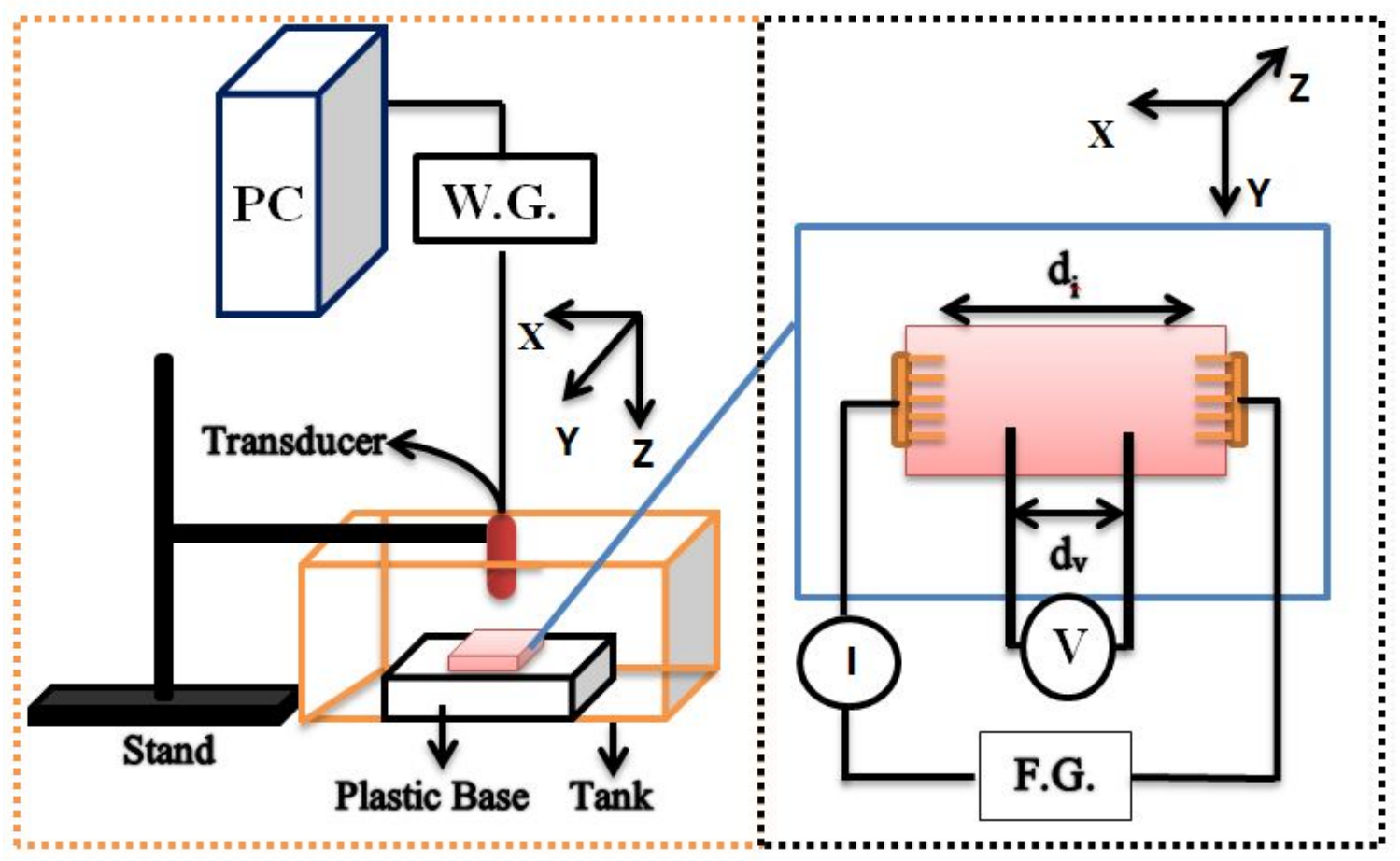

Figure 3.1: Experimental setup for single element transducer experiments

Electrodes were kept in contact with the samples such that their terminals were across the longest dimension (across X-axis in the figure). A Function Generator (F.G.)[DS335, Stanford Research Systems] was used in order to apply square waveforms at the frequency $0.025 \mathrm{~Hz}$. A piezoelectric transducer $\left(10 \mathrm{MHz}\left(F_{c}\right.\right.$ ) focal length of $0.75^{\prime}$ and diameter of $0.5^{\prime}$ or a $5 \mathrm{MHz}\left(F_{c}\right)$ unfocused) was placed very close ( $1 \mathrm{~cm}$ to $2 \mathrm{~cm}$ ) to the top surface of the tissue sample in order to constantly acquire RF data. The firing of the transducer was performed by an ultrasonic pulser-receiver (Olympus - 5077PR, W.G. in the figure). The transmission of the ultrasound wave was along the Z-axis. Constant RF data was acquired at a sampling rate of $200 \mathrm{MHz}$ by a 14bit data acquisition card. The recorded data was analysed using an algorithm created in Matlab $^{T M}$. The data acquired was averaged 500 times every second and each A-line comprised of 14972 sampling points. The sampling rate of acquisition for experiments with linear array transducers was $200 \mathrm{MHz}$ which translates to a sample spacing of $5 \mathrm{~ns}$. The algorithm developed used interpolation to estimate the time delay estimates. The signals were interpolated with a step size of 0.1. Therefore, the sub sample delays estimated by the algorithm can be as low as $0.5 n s$. The spatial resolution of the system depends on the wavelength of the ultrasound and the windowing size used. The temporal resolution was adjusted to 1-2s depending on the pulse repitition frequency and averaging used. Electric fields varying from $0.25 \mathrm{~V} / \mathrm{cm}$ to $1.6 \mathrm{~V} / \mathrm{cm}$ were applied to the samples. 
BE - Before application of Electric field.

E - During application of Electric field.

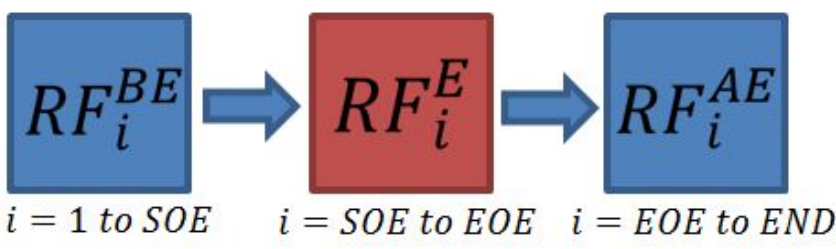

AE - After application of Electric field.

$$
\begin{gathered}
\text { SOE = Start of Electric Field application } \\
\text { EOE = End of Electric Field application } \\
\text { END }=\text { End of experiment }
\end{gathered}
$$

Figure 3.2: Experimental procedure for single element transducer experiments

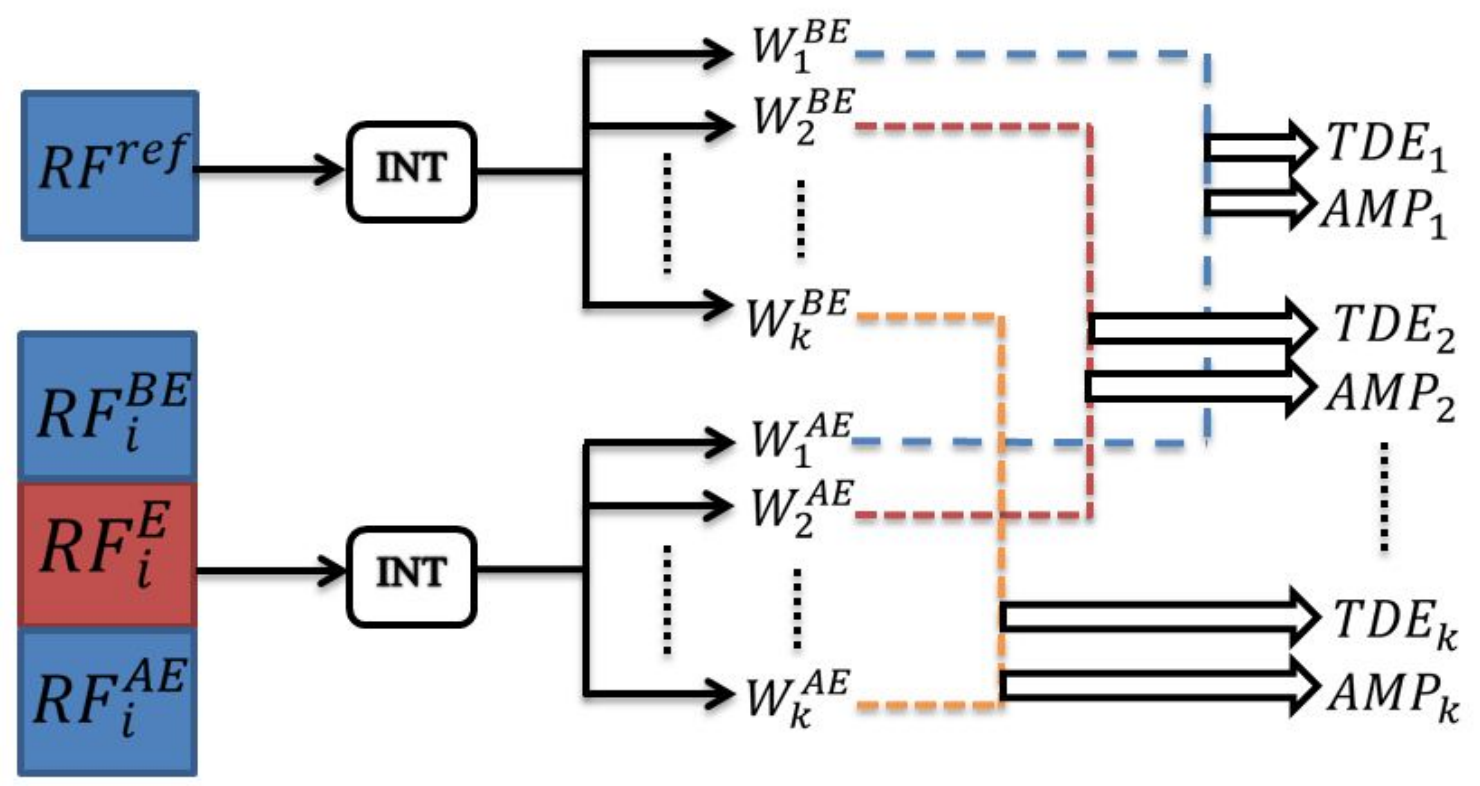

Figure 3.3: Description of algorithm being used 


\subsubsection{Brief description of the algorithm}

An arbitrary referencesignal $R F^{r e f}$ was chosen from the signals acquired before the application of electric field. The signals were then interpolated(INT in figure 3.3) using the interp 1 function provided in Matlab in order to better estimate time delays(and to estimate subsample delays). The RF signal was segregated into small windows $W_{k}^{B E} \& W_{k}^{E}$ (approximately 1 to $2 \lambda \mathrm{s}$ ). These individual windows were compared with their respective windows before and after the application of electric fields. While applying cross correlation, time delay estimates $T D E_{k}$ were calculated. The peak to peak amplitude changes $A M P_{k}$ were also analysed for each of the windows(see figure $3.2 \& 3.3$ ).

$$
R(\tau)=\int W_{k}^{r e f}(t) \cdot W_{k}^{E}(\tau+t) d \tau
$$

Equation 3.1 defines cross correlation for the windowed signal. The delay estimate for a particular window can be found by:

$$
T D E_{k}=\left.\tau\right|_{\max (R)}
$$

\subsection{Experiments with Linear Array Transducers}

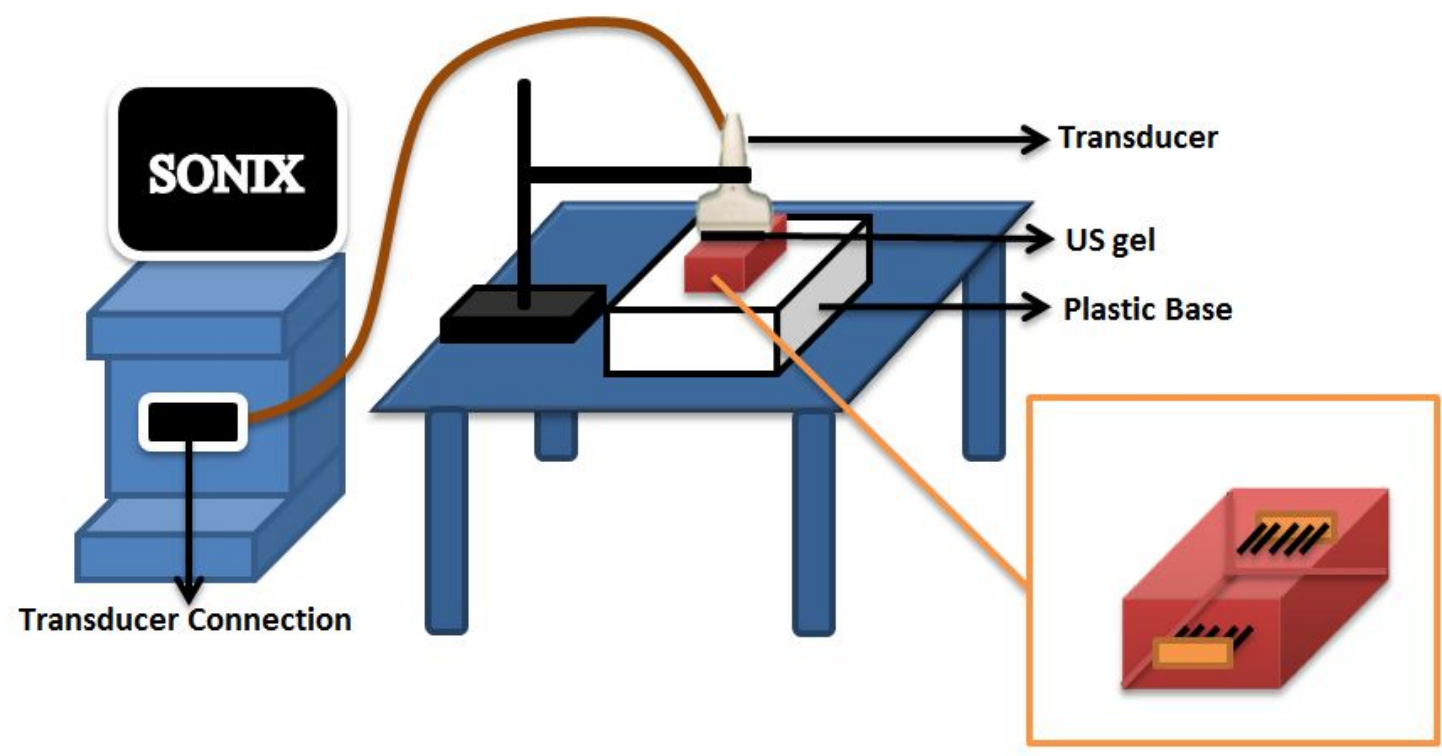

Figure 3.4: Experimental procedure for linear array transducer experiments

The 128-element linear-array ultrasound transducer was attached to its base unit on the Sonix RP[Ultrasonix, BC, Canada] ultrasound system(As shown in figure 3.4). Constant RF data was recorded while taking images in B-mode of the ultrasound system. The probe was electrically insulated from the sample. The center frequency of the transducer was $5 \mathrm{MHz}$ and the focus was arranged such that it was 
within the limits of our tissue sample. The data was acquired at a sampling rate of $40 \mathrm{MHz}$ and B-mode images were obtained at a rate of 1 FPS (frames per second). The experiment was divided into three major steps. The RF data and images were acquired simultaneously, before the application of electric field, and this data becomes the reference. The second step involves application of voltage across the electrodes. New RF data and images were acquired simultaneously. Ultimately, the voltage was removed and the data is acquired for another minute.

\subsection{Data Analysis}

After performing the experiments, data analysis was performed in order to visualise the changes occurring in the received echo signals. The B-mode ultrasound image obtained from Sonix RP was produced by congregating together various $\mathrm{A}$-lines $\left(A_{1}, A_{2} \ldots A_{j}\right)$ of $\mathrm{RF}$ data containing data points corresponding to the depth being imaged. Raw data was obtained and a single RF line was chosen from the data before voltage application. This RF line represents the received echo amplitude from the spatial extent of experimental setup in the transducers axial direction. Small windows $\left(W_{1}, W_{2} \ldots W_{k}\right)$ (approximately about 1 or 2 times the wavelength) were chosen within the $\mathrm{A}$ line to analyse the changes. Amplitude changes were measured by using an algorithm to find the peaks within the windows and observing the changes occurring in the peak to peak amplitude. Signal shifts(TDE) within the windows chosen are measured by a cross correlation algorithm. The changes are then monitored over a long period of time starting from the beginning of data acquisition to the end of data acquisition. Figure 3.5 shows the approach taken for forming images. TDE images are formed by analysing windows within every A-line.

\subsubsection{Observing the changes in slow-time within various windows}

To observe the changes in slow time, the signal is segregated into two parts. The first part is defined as the slow-time shifting trend as shown in figure 3.6 . The second part represents the fluctuations in the shifting during the application of the electric field. This part is termed as slow-time shifting fluctuations. These fluctuations are obtained after detrending the data within the duration of the applied electric field. The trend can be viewed as the DC component in the shifting changes and the fluctuations can be viewed as the AC component. The DC component provides information about the expansion of the tissue whereas the $\mathrm{AC}$ component provides information about the correspondence to the applied electric fields.

A similar approach was followed for the fast-time shifting trends. The shifting trends are discussed in more detail in chapter 4 .

\subsubsection{Analyzing fluctuations in slow-time shifting}

To analyze the fluctuations in slow-time shifting, the fluctuations are first obtained as described in section 3.6.1. Fourier transform is then applied to these fluctuations and the frequency content of the fluctuations is examined. The amplitude of the frequency spectrum was used to represent the correspondence to the applied electric field. 

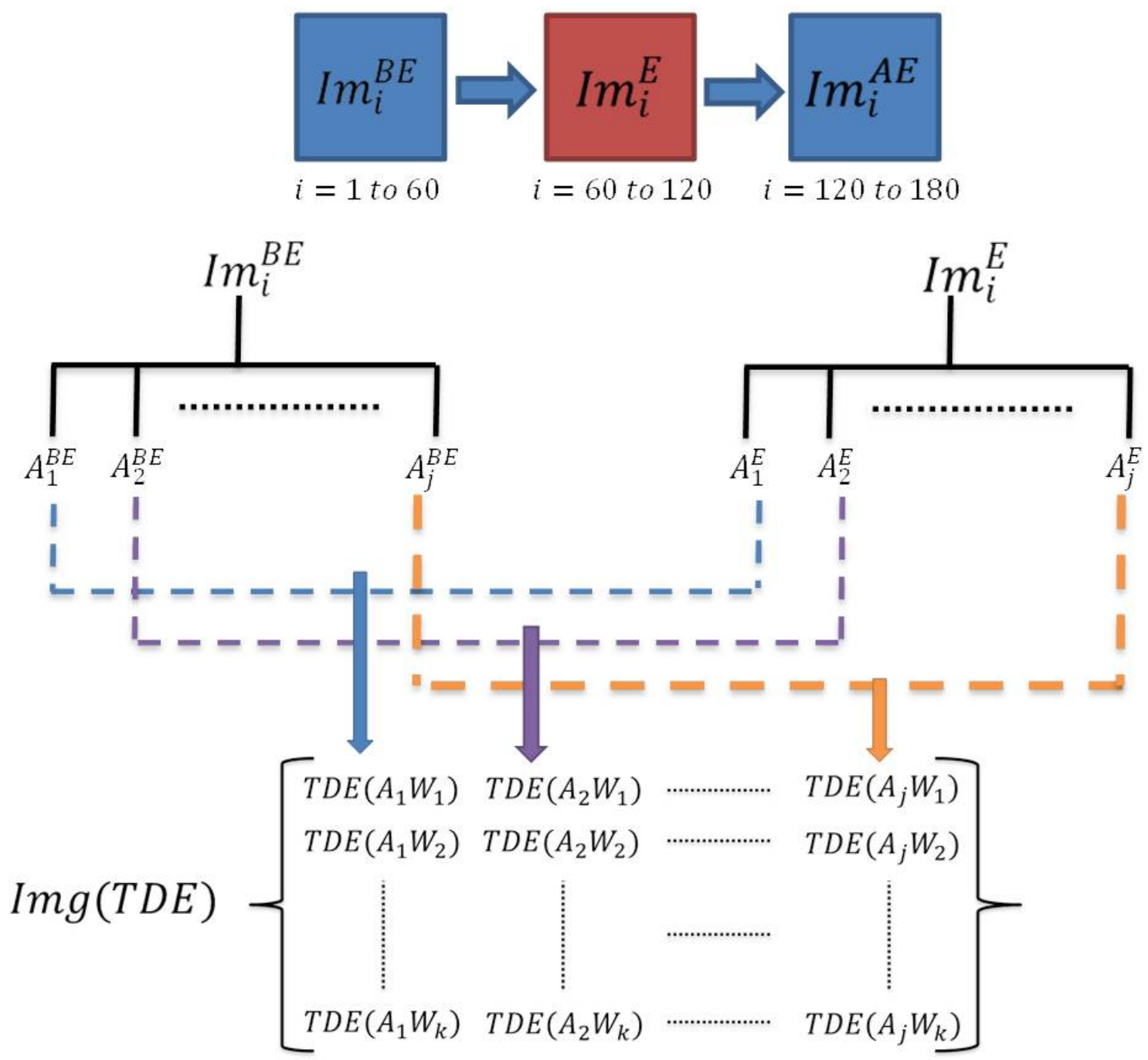

Figure 3.5: Procedure for analysing B-mode images 

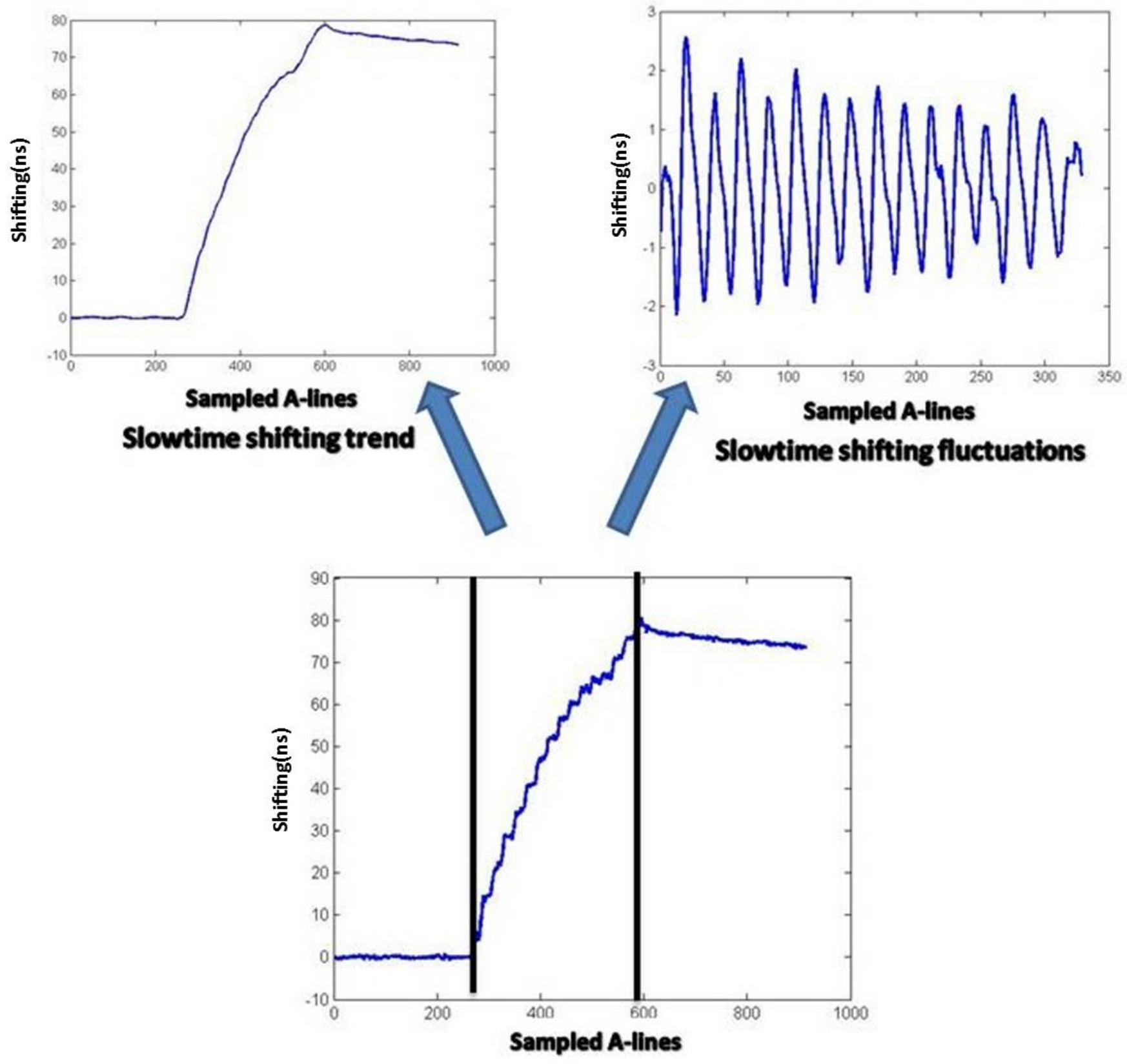

Figure 3.6: A schematic for analysing the slow time shifting 


\subsubsection{Evaluating the strain generated}
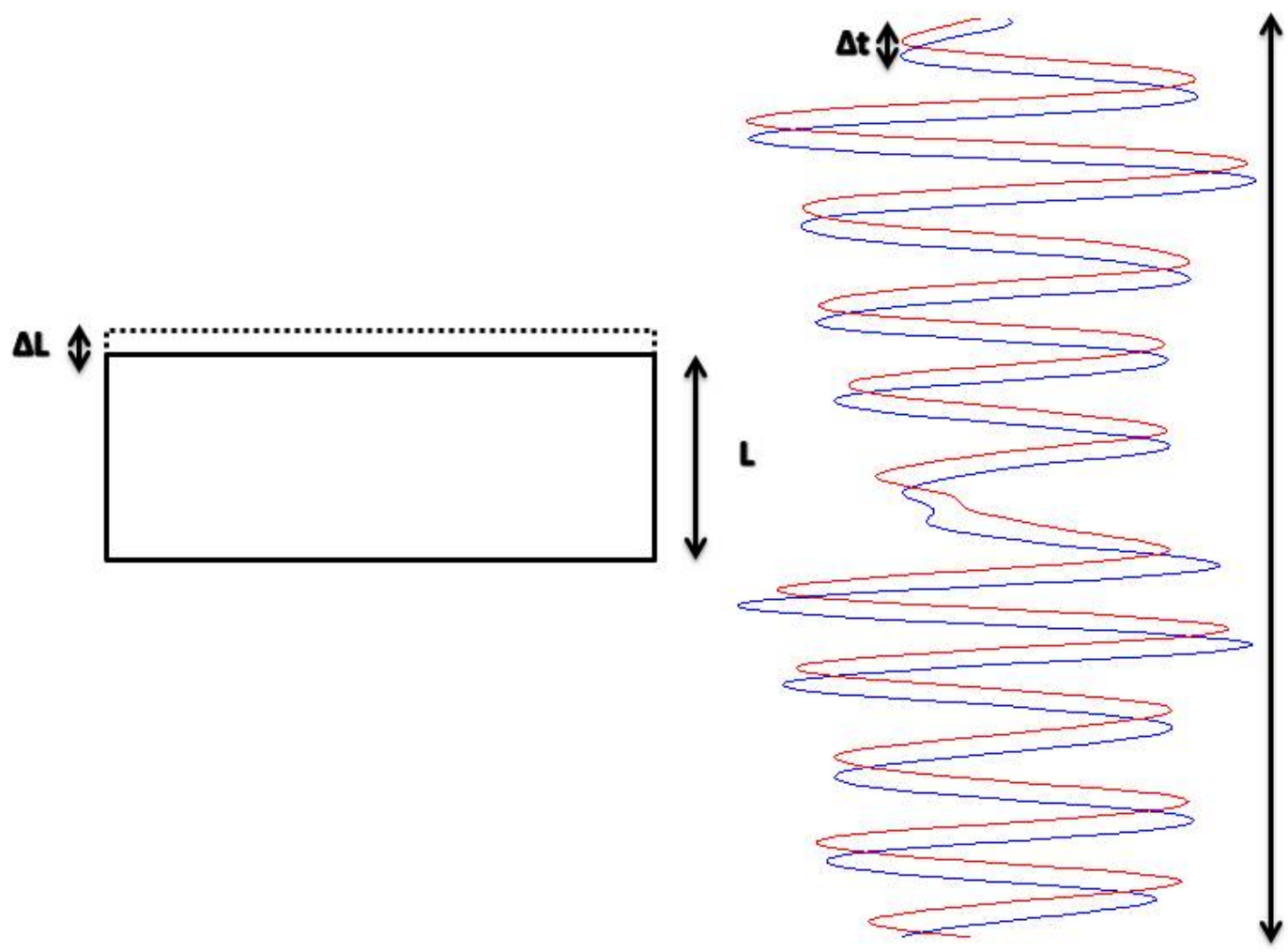

Figure 3.7: A schematic for analysing strain

Strain in simple terms can be defined as

$$
\frac{\text { Change in length }(\Delta L)}{\text { Original length }(L)}
$$

The strain estimation on ultrasound signals can be done in a similar fashion. Figure 3.7 shows a schematic describing the calculation of strain for the ultrasound signals. Strain is generally donated in terms of percent change, and for ultrasound signals, the strain can be defined as

$$
\frac{\text { shifting in the front window - shifting in the rear window }}{\text { Difference in arrival time of the front and the rear windows } / 2}
$$




\subsubsection{Overlay for B-mode images}

Figure 3.8 shows an example of overlay generated by using a grayscale B-mode image(porcine tissue) and an image obtained by time delay estimates. The colorscale is represented on the right of the figure. The TDE image is made transparent and overlayed over the normal B-mode image.

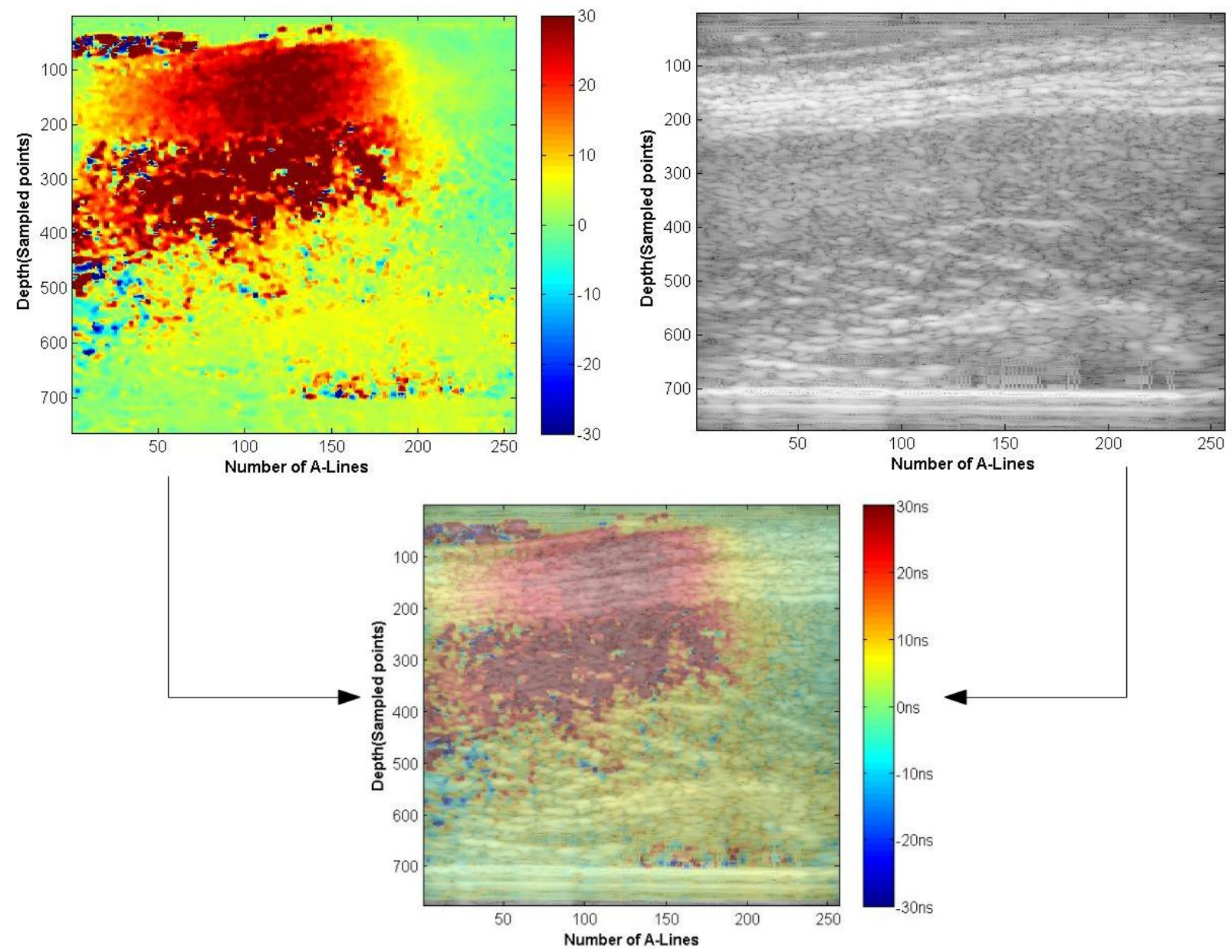

Figure 3.8: Generating B-mode \& TDE overlays 
Chapter 4

\section{Results}




\section{Organization of the chapter}

The results obtained are discussed and analysed in this chapter. To begin, the results are arranged in a general presentation which provides an insight on the slow-time shifting, fast-time shifting and the strain generated in phantoms with and without scatterers. The results are then compared to observe the dependence of EIMC on electric fields. Slow time shifting fluctuations and slow-time shifting rate were observed at different applied electric fields. The dependence of the EIMC on the concentration of gelling agents was observed next. This section also discussed the variability in the experimental data. The following section presents the dependence on the salt concentration and temperature changes. At the end of this chapter, results obtained from experiments performed on a linear array are shown.

\subsection{General presentation of the results}

\subsubsection{Phantom Nomenclature}

Table 4.1: Phantom nomenclature and contents

\begin{tabular}{|c|c|c|c|}
\hline Name of the Phantom & Gelation Agent & Additive I & Additive II \\
\hline Phantom G-I & Gelatin $3 \% w / v$ & Salt $2 \% w / v$ & Graphite $5 \% w / v$ \\
\hline Phantom G-II & Gelatin $6 \% w / v$ & Salt $1 \% w / v$ & Graphite $7 \% w / v$ \\
\hline \hline Phantom G-III & Gelatin $6 \% w / v$ & Salt $2.5 \% w / v$ & Graphite $0 \% w / v$ \\
\hline \hline Phantom A-I & Agar $0.5 \% w / v$ & Salt $0.5 \% w / v$ & Graphite $0 \% w / v$ \\
\hline Phantom A-II & Agar $1 \% w / v$ & Salt $0.5 \% w / v$ & Graphite $5 \% w / v$ \\
\hline
\end{tabular}

Table 4.1 displays selected phantoms which are chosen for discussing the temporal changes occurring due to the application of electric fields. Comparisons between them, and other phantoms not displayed in the table, would follow after the preliminary analysis of the shifting.

\subsubsection{Gelatin Phantoms}

\section{Conductivity of gelatin phantoms}

Figure 4.1 shows the change in conductivity against the salt concentration. An example of such values of conductivity are depicted in figure 2.5. Adding salt to the phantom increases the conductivity and the values of conductivity bear similarities to saline conductivities at the same concentrations. 

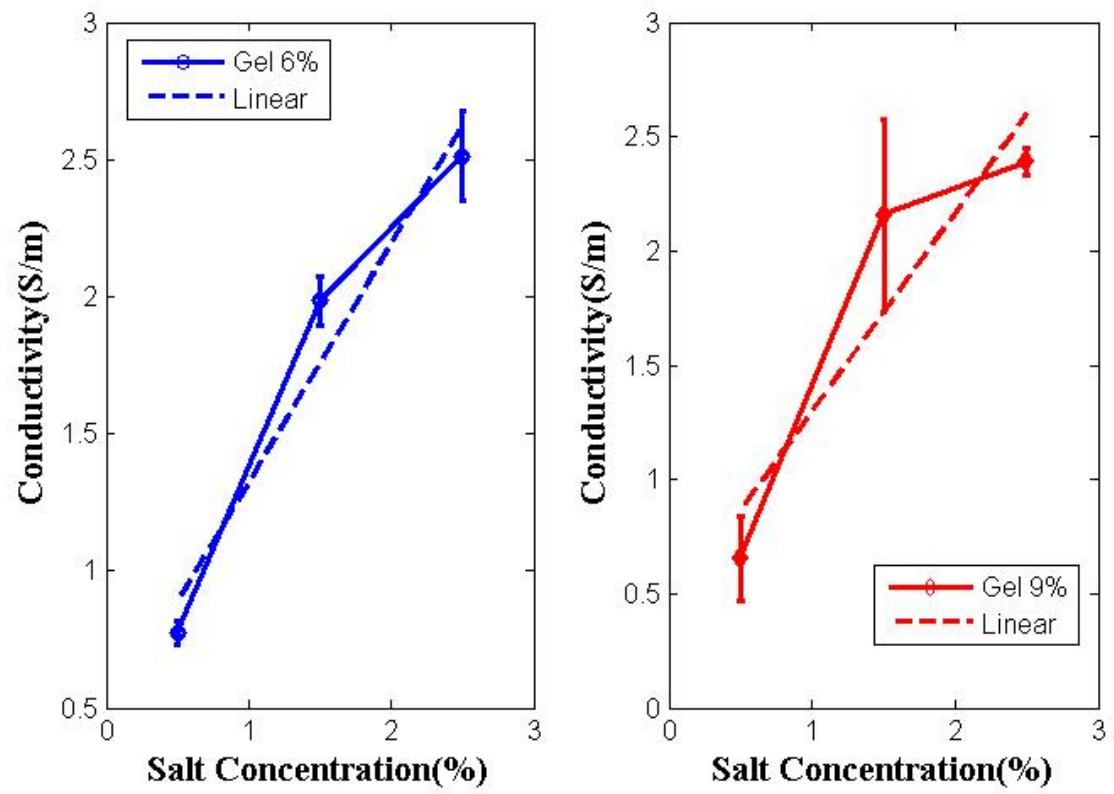

Figure 4.1: Conductivity of various concentrations of $\mathrm{NaCl}$ in gelatin phantoms-experimental results.

\section{Shifting in signal windows with scatterers in the phantom}

\section{Phantom Type G-I}

Experiment parameters

Frequency of applied electric field : $0.025 \mathrm{~Hz}$

Magnitude of the electric field : $\quad 3.5 \mathrm{~V}$

Start of application : $\quad 270 \mathrm{~s}$

End of application $\quad: 598 \mathrm{~s}$

Total acquisition : $\quad 914 \mathrm{~s}$

The phantom was prepared as described in the methods section. Using the experimental apparatus, electric field was applied to the phantom and changes occurring in the recorded ultrasound signals were observed. The results of this experiment are shown in figure 4.2. Part $a$ of the figure represents an A-line formed from the reflected echoes through the phantom. Part $b$ of the figure the shifting of the front and the rear boundaries of the phantom versus slow time. As seen, it was observed that the shifting near the front boundary was the highest and the rear boundary shifting stayed close to 0ns. The difference in the shifting denotes a general expansion of the phantom towards the face of the transducer. The shifting of the RF signals is mainly due to the expansion of the sample. The shifting profile of the phantom, as depicted in part $c$, displayed the shifting through the phantom at different depths. The total depth of the phantom was around $1.5 \mathrm{~cm}$ and the shifting of the signal decreased with the increase in depth. Strain profile of is displayed in part $d$ of the figure. The strain as well as the shifting portrayed a fluctuating 


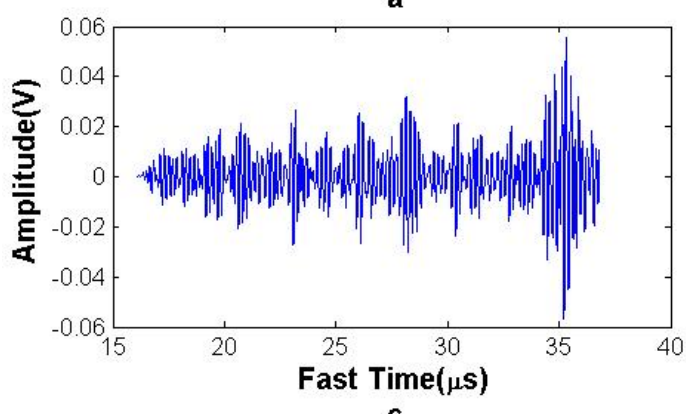

C

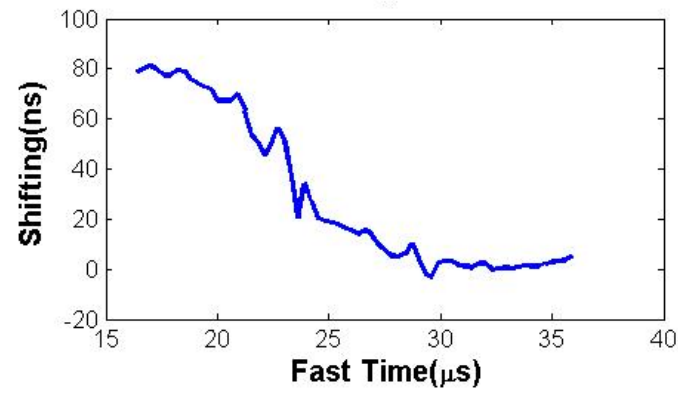

b

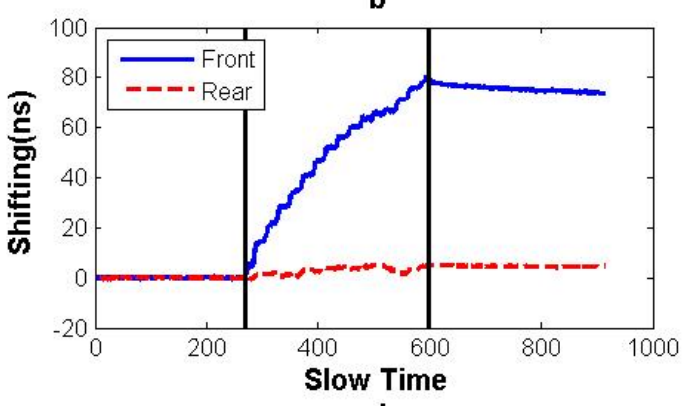

d

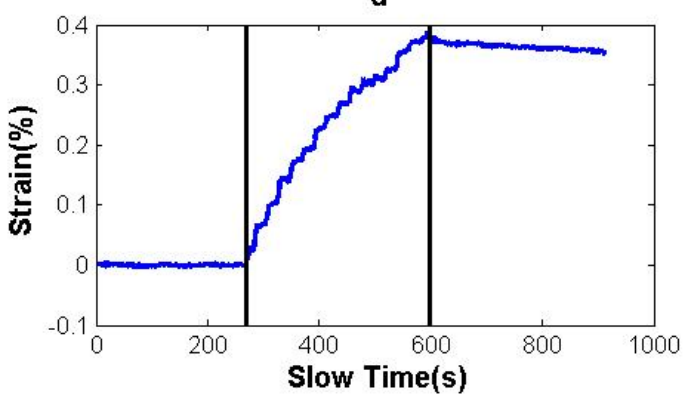

Figure 4.2: (a) Ultrasound A-line as recorded from the phantom G-I. (b) Shifting of the front and the rear boundaries of the phantom G-I during the slow time (black lines represent application of voltage across the phantom). (c) Shifting of the whole phantom G-I from the front to the rear boundary of the phantom at the end of the electric field application. (d) Strain generated due to motion of the phantom G-I during the application of current. 
pattern which corresponded to the frequency of the applied electric field.

\section{Phantom Type G-II}
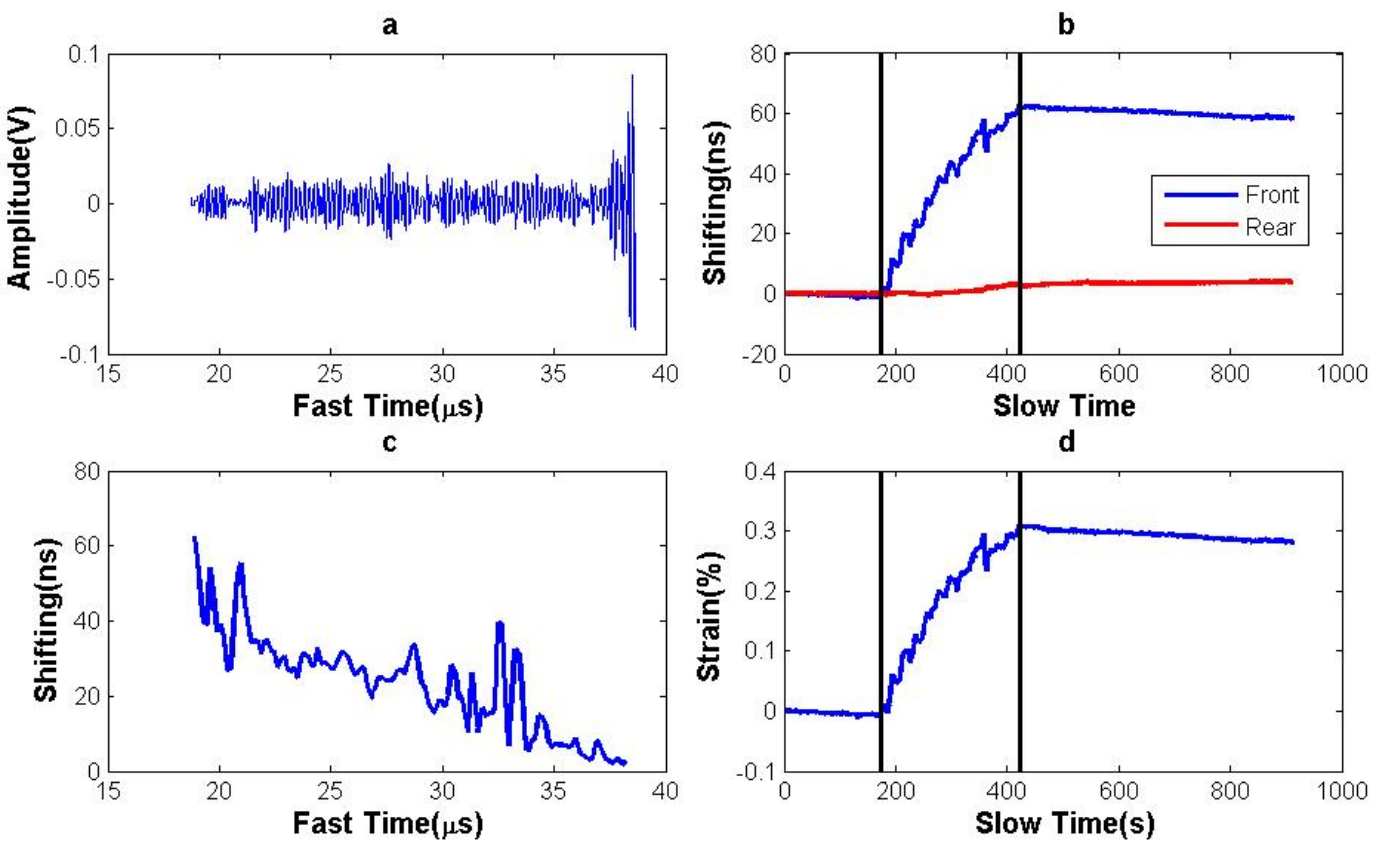

Figure 4.3: (a) Ultrasound A-line as recorded from phantom G-II. (b) Shifting of the front and the rear boundaries of the phantom G-II during the slow time(black lines represent application of voltage across the phantom). (c) Shifting of the whole phantom G-II from the front to the rear boundary of the phantom at the end of the electric field application. (d) Strain generated due to motion of the phantom G-II during the application of current. 
Experiment parameters

Frequency of applied electric field : $0.025 \mathrm{~Hz}$

Magnitude of the electric field : $\quad 3.5 \mathrm{~V}$

Start of application : $\quad 175 \mathrm{~s}$

End of application $\quad: \quad 423 \mathrm{~s}$

Total acquisition : $\quad 911 \mathrm{~s}$

Since graphite powder was used as scatterers for this experiment, the signal from interior parts of the phantom was strong(Figure 4.3(a)). As noticed in part(b), application of electric field gradually increased the time delay between the reference signal and the signal under examination. The blue line represents the shifting of the signal near the front boundary of the phantom. The red line, which represents the rear boundary of the phantom, shows that signals arriving from the rear part of the phantom observe negligible shifting with respect to the front boundary. The delay estimates along the axial direction of the transducer is shown in part(c). It clearly displays the expansion of the phantom towards the face of the transducer. The maximum shifting observed was $60 \mathrm{~ns}$ which translates to an increase of $90 \mu \mathrm{m}$ in the axial direction. The strain is calculated and is displayed in part (d).

\section{Shifting in the signal windows without scatterers in the phantom}

\section{Phantom Type G-III}

Experiment parameters

Frequency of applied electric field : $0.025 \mathrm{~Hz}$

Magnitude of the electric field : $10 \mathrm{~V}$

Start of application : $\quad 300 \mathrm{~s}$

End of application : $\quad 910 \mathrm{~s}$

Total acquisition : $1207 \mathrm{~s}$

The electric field intensity is higher in this experiment and hence the shifting of the front boundary is higher in comparision to other studies. The slow time response of the phantom is a bit differentiated as compared to other studies. There can be two regions observed where the gradient changes from high to low. 


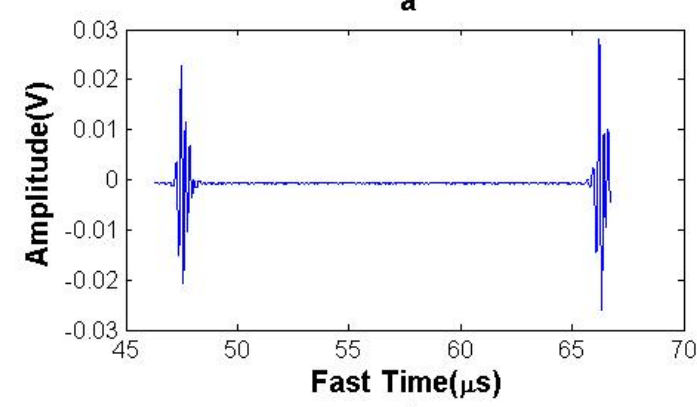

c

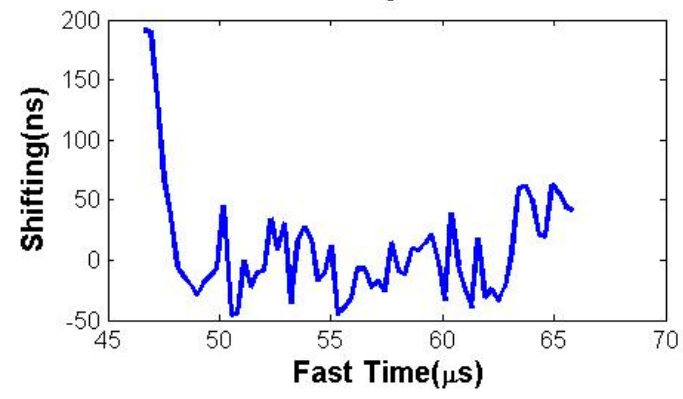

b

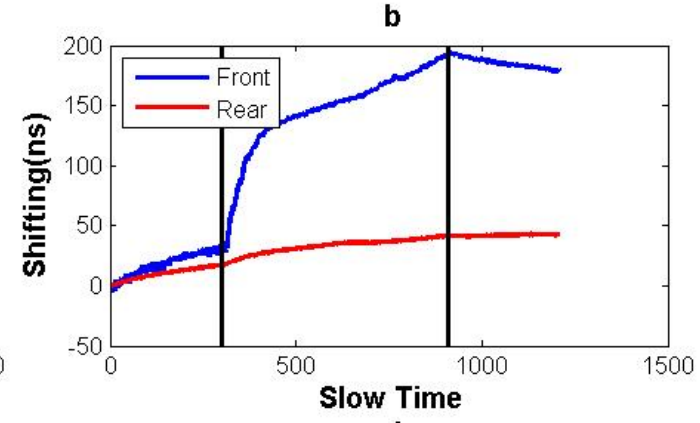

d

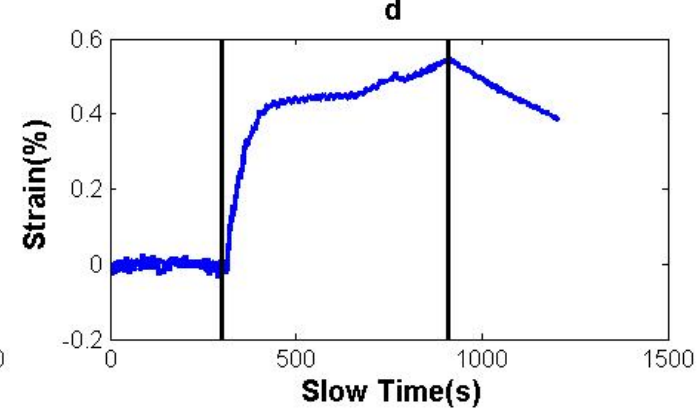

Figure 4.4: (a) Ultrasound A-line as recorded from phantom G- III. (b) Shifting of the front and the rear boundaries of the phantom G-III during the slow time (black lines represent application of voltage across the phantom). (c) Shifting of the whole phantom G-III from the front to the rear boundary of the phantom. (d) Strain generated due to motion of the phantom G- III during the application of current. 


\subsubsection{Agar Phantoms}

Shifting changes in signal windows without scatterers in the phantom

\section{Phantom Type A-I}

Experiment parameters

Frequency of applied electric field : $0.025 \mathrm{~Hz}$

Magnitude of the electric field : $\quad 4 \mathrm{~V}$

Start of application : $221 s$

End of application $\quad: \quad 445 \mathrm{~s}$

Total acquisition $\quad$ : $692 \mathrm{~s}$
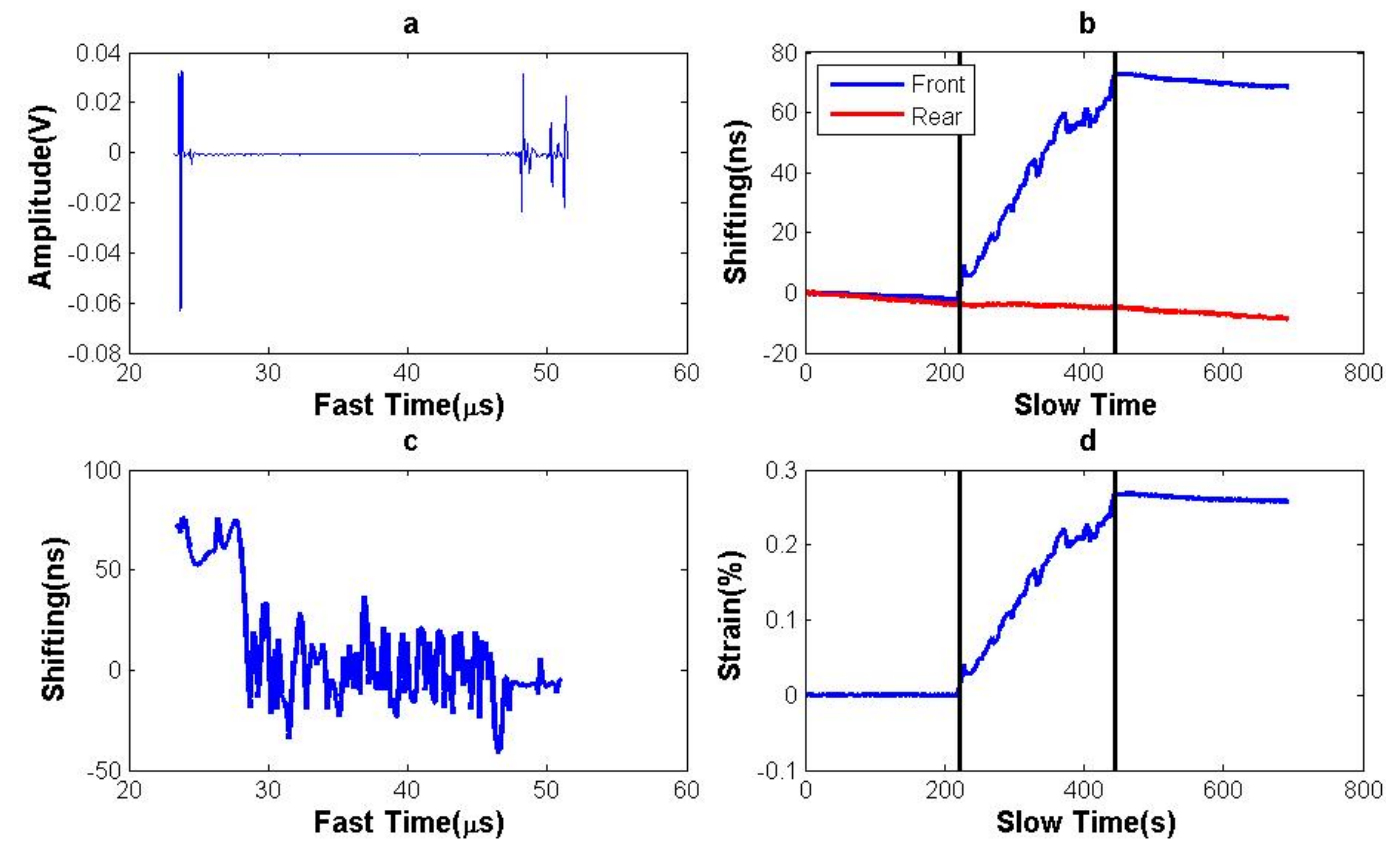

Figure 4.5: (a) Ultrasound A-line as recorded from phantom A-I. (b) Shifting of the front and the rear boundaries of the phantom A-I during the slow time(black lines represent application of voltage across the phantom). (c) Shifting of the whole phantom A-I from the front to the rear boundary of the phantom. (d) Strain generated due to motion of the phantom A-I during the application of current.

Since the phantom A-I does not have any scatterers embedded inside, the echoes at the front and the rear boundaries are prominent and there are not much of interior echo signals recorded in the A-line (Figure 4.5 (a)). Due to this low interior echo signals, the CC algorithm cannot resolve proper correlation values for the windows which represent the interior parts of the phantom(Figure 4.5 (c)). The front and the rear boundary reflections are considered to be important locations for the $\mathrm{CC}$ algorithm and the same trends are observed as noted in the previous phantom studies. The strain was calculated by using the shifting of the front and the rear boundaries. 
Shifting changes in signal windows with scatterers in the phantom

Phantom Type A-II

Figure 4.6 shows an agar phantom with scatterers embedded. Part(a) shows the increased scattering from within the sample. Phantoms with graphite scatterers embedded within always performed better in terms of the fast-time shifting.
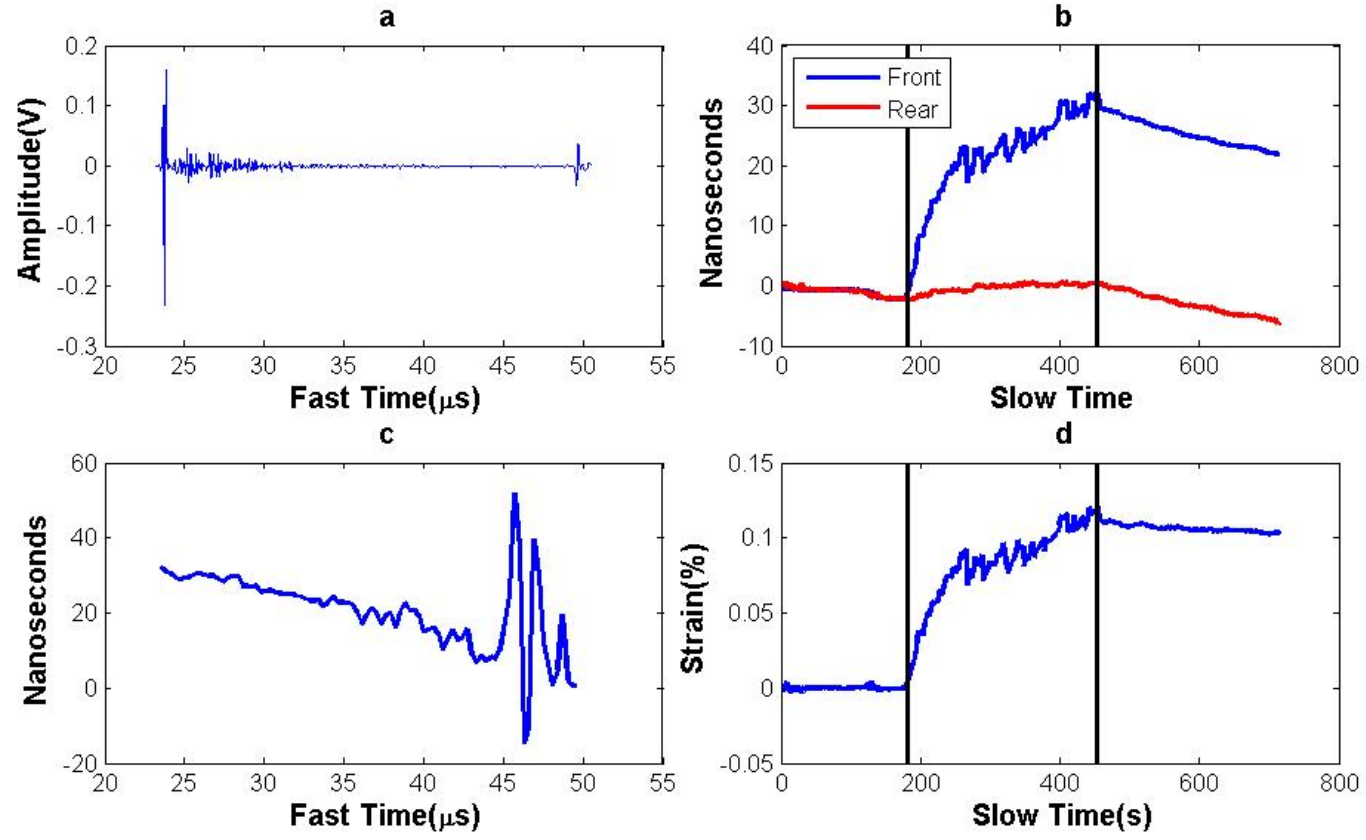

Figure 4.6: (a) Ultrasound A-line as recorded from phantom A-II. (b) Shifting of the front and the rear boundaries of the phantom A-II during the slow time(black lines represent application of voltage across the phantom). (c) Shifting of the whole phantom A-II from the front to the rear boundary of the phantom. (d) Strain generated due to motion of the phantom A-II during the application of current.

\subsection{Fast-time shifting, slow-time shifting and the amplitude changes occurring within the signals}

\subsubsection{Fast-time signal shifting comparison - different instances in slow time}

Figure 4.7- 4.10 depict the fast time shifting trend change during various instances of time. The first legend entry for every plot indicates the fast-time shifting at the onset of application and the last entry is in near proximity of the removal of electric field. The trend of shifting being least (almost flat) at the start of application and highest near the end of the application. It is also observed that some features 


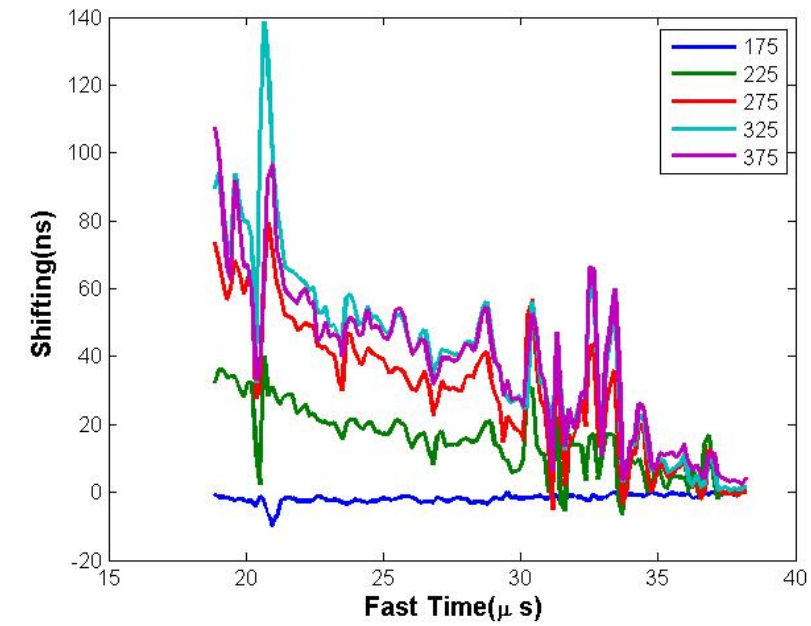

Figure 4.7: Fast-time shifting trend changes of a gel $6 \%$ sample occurring during slow time instances

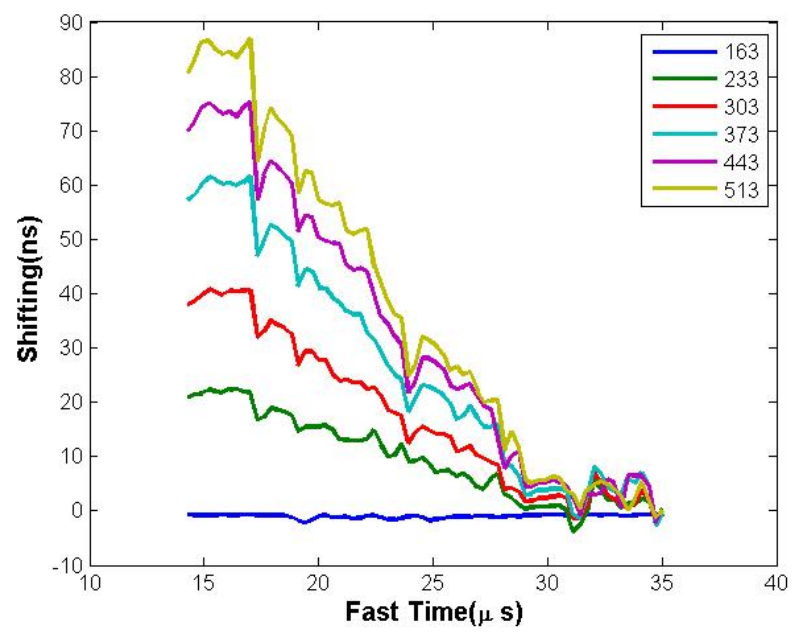

Figure 4.9: Fast-time shifting trend changes of a gel $9 \%$ sample occurring during slow time instances

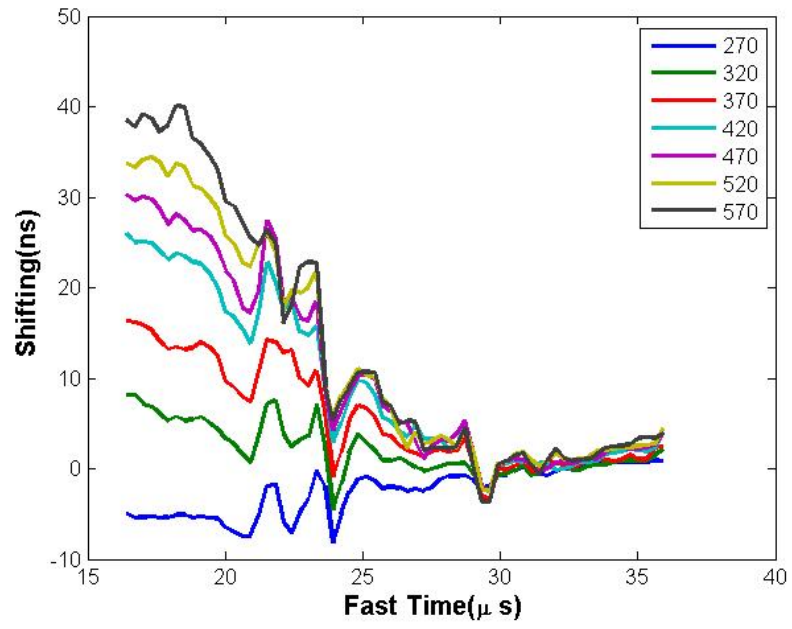

Figure 4.8: Fast-time shifting trend changes of a gel $3 \%$ sample occurring during slow time instances

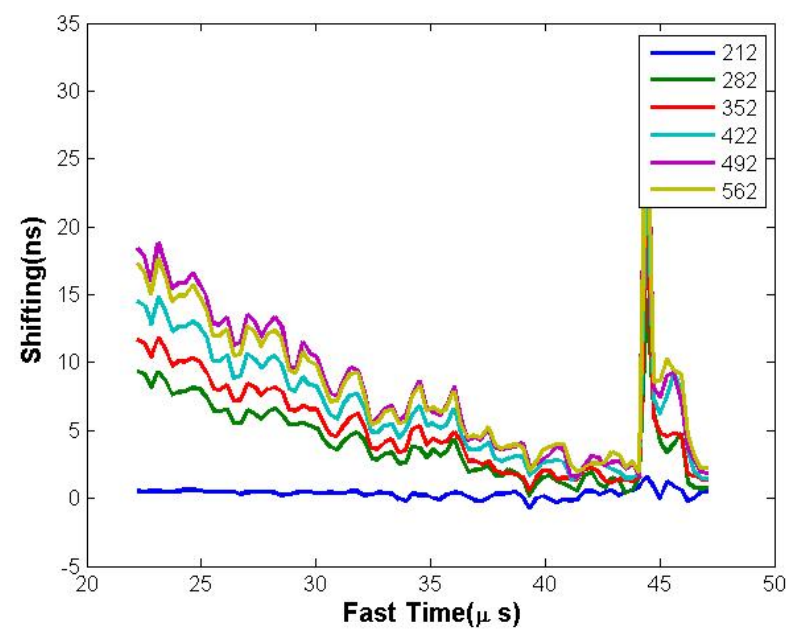

Figure 4.10: Fast-time shifting trend changes of an agar $1 \%$ sample occurring during slow time instances 
become more apparent towards the end of the application cycle. These features are unique to individual phantoms.

Front boundary and the rear boundary of the samples were identified and a global strain was estimated between them. Rear boundary of all the samples constantly remained stable and there was no shifting observed on the rear face of the sample. The selection of the front and the rear boundaries are important as they characterize the strain profile of the sample and aid in explaining the phenomenon.

\subsubsection{Slow-time signal shifting comparison - different windows within the same signal}

Slow time shifting for different windows within the same signal exhibits a unique response for every window. The shifting near the front boundary is the highest and it decreases as the window reaches the rear boundary. Figures $4.11-4.14$ display the slow time shifting changes within different windows. These plots show depth dependent changes occurring within the samples. The first entry of the legend displays a window which lies near the front boundary of the sample. The following entries are consequently selected windows at increasing depths of the sample ending in a window which in near the rear boundary.

Apart from the general linear trend of the shifting, there were a few experiments which showed multiple trends. The slope of shifting was observed to be higher near the start of the application of the

electric field and it gradually plateaued. These effects were observed in experiments where the electric field intensity was higher $(1.6 \mathrm{~V} / \mathrm{cm})$ and a few experiments with agar phantoms. 


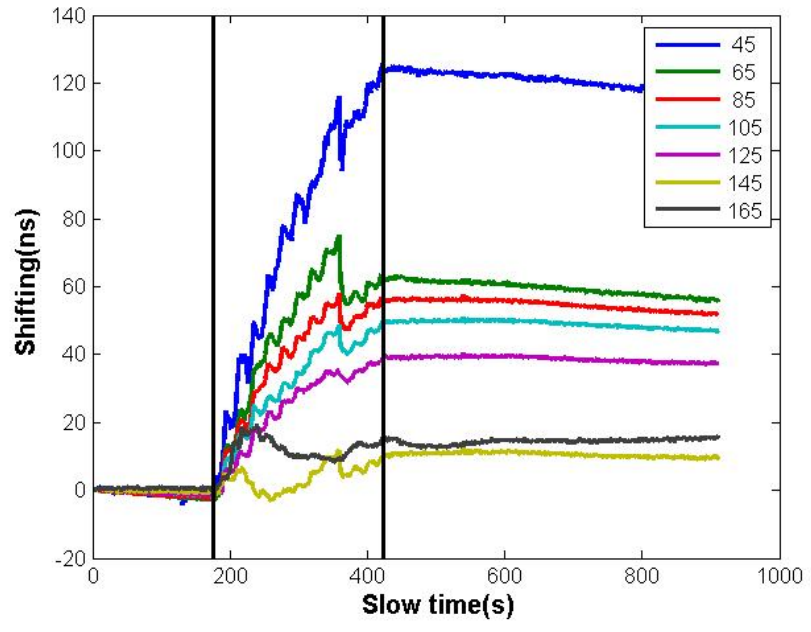

Figure 4.11: Slow time shifting variations in individual windows of a gel $6 \%$ sample

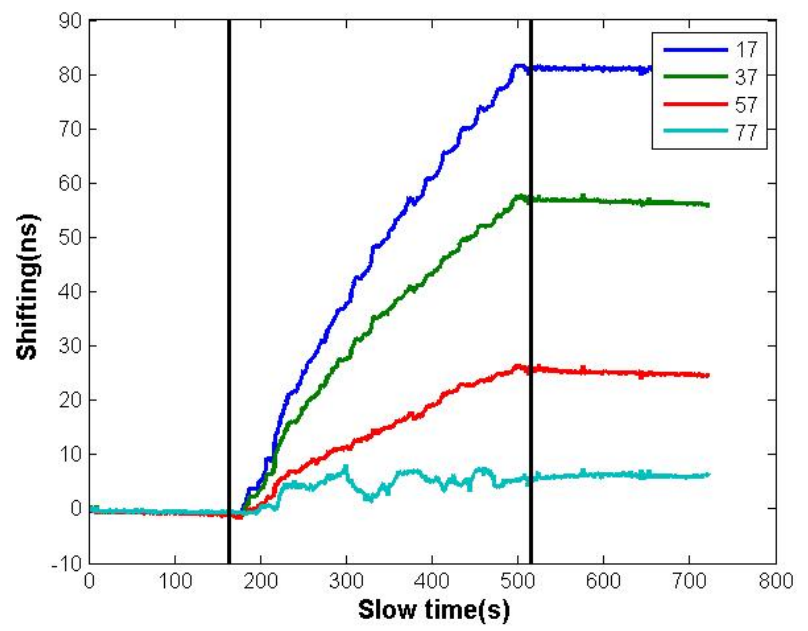

Figure 4.13: Slow time shifting variations in individual windows of a gel $9 \%$ sample

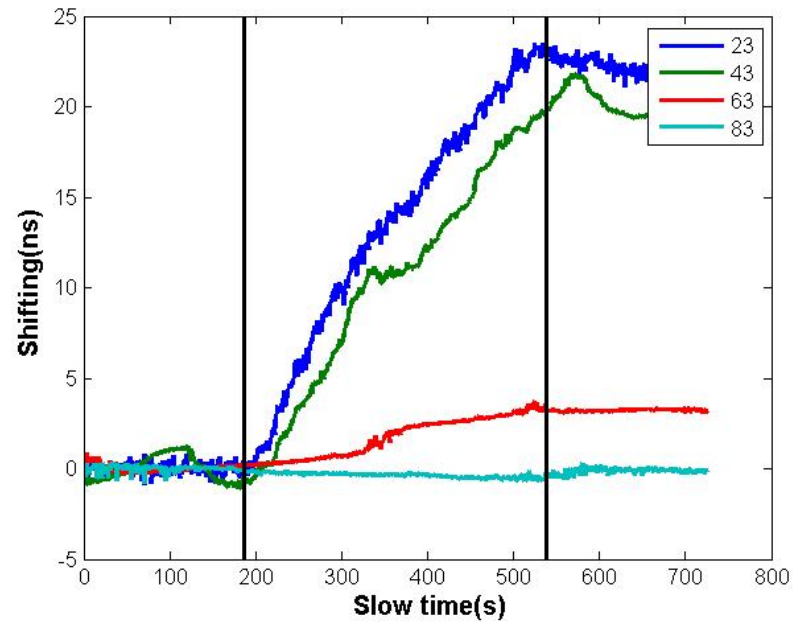

Figure 4.12: Slow time shifting variations in individual windows of a gel $3 \%$ sample

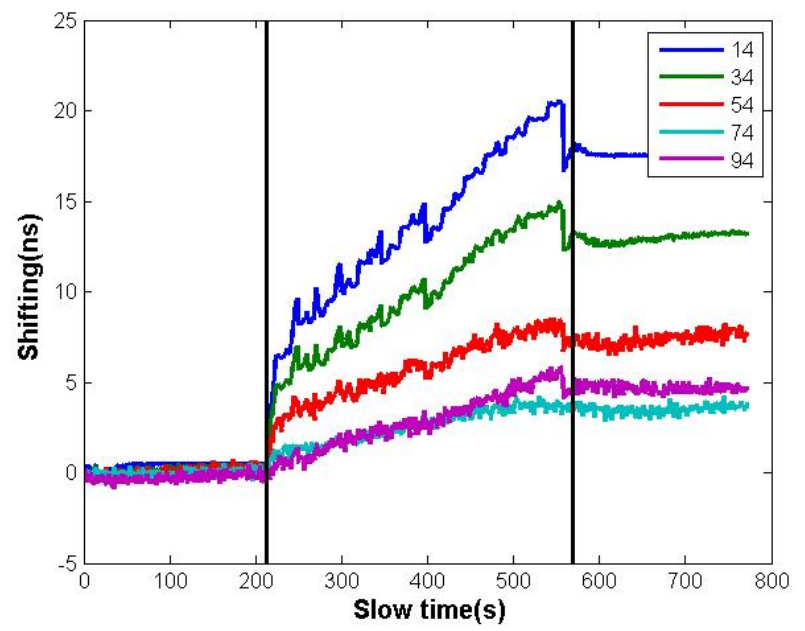

Figure 4.14: Slow time shifting variations in individual windows of an agar 1\% sample 


\subsubsection{Amplitude changes due to the application of electric fields}

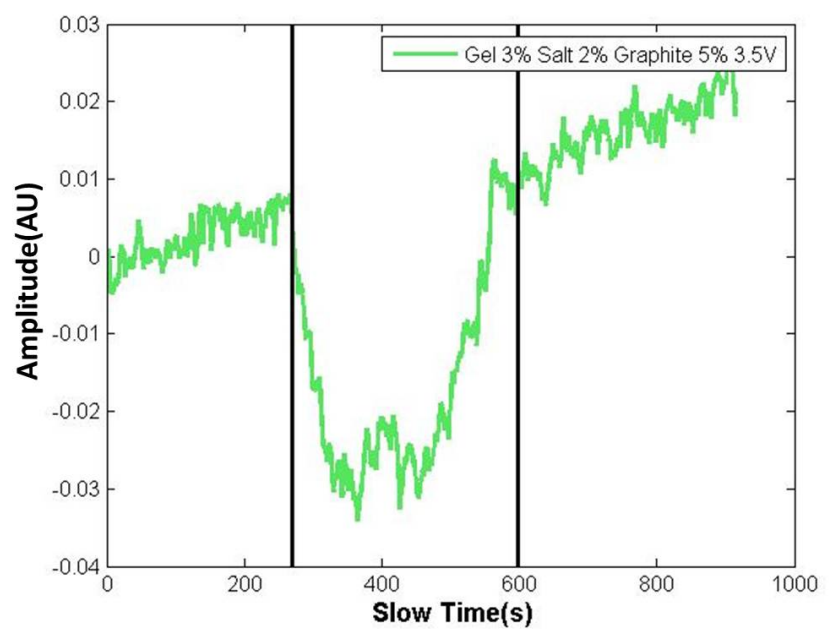

Figure 4.15: Amplitude changes near the front boundary of a gel $3 \%$ sample

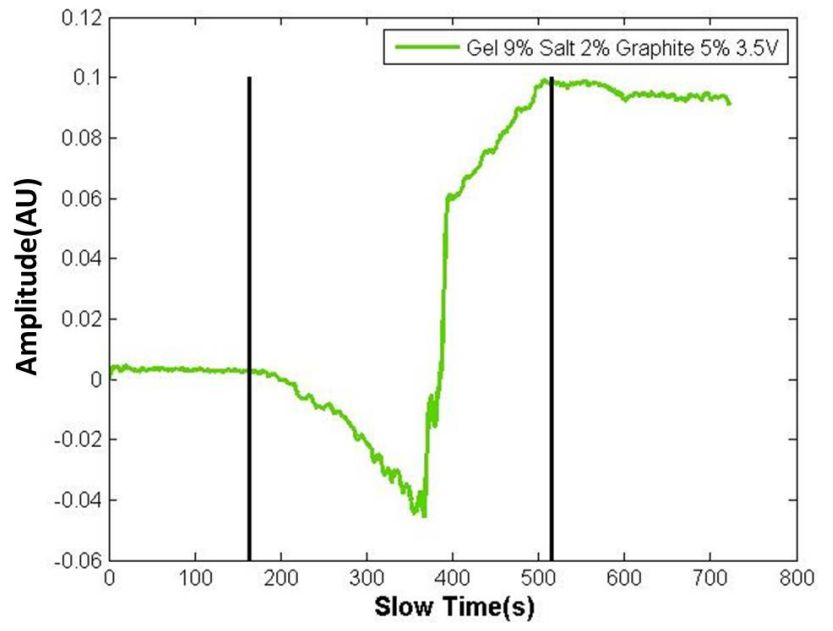

Figure 4.17: Amplitude changes near the front boundary of a gel $9 \%$ sample

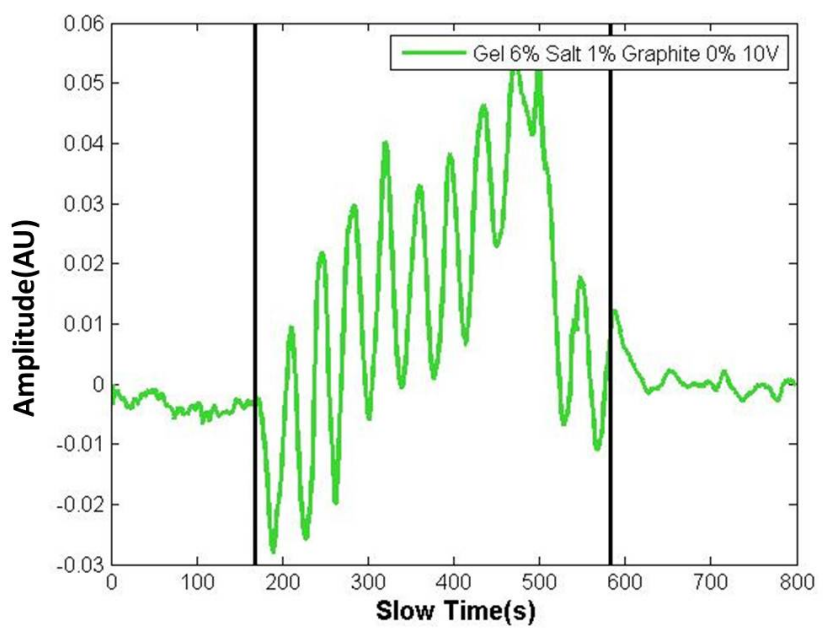

Figure 4.16: Amplitude changes near the front boundary of a gel $6 \%$ sample

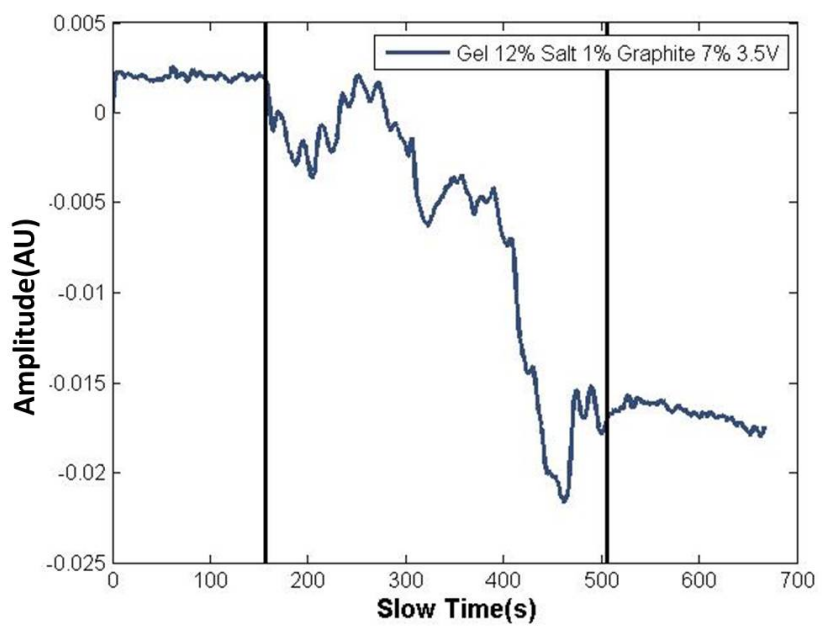

Figure 4.18: Amplitude changes near the front boundary of a gel $12 \%$ sample

The amplitude changes observed within the windowed signals did not correspond to a particular trend. Before and after the application of electric field, the amplitude changes were almost negligible. Some changes before the application were attributed to the environmental noise and experimental conditions. The black lines represent the onset and removal of electric field. The figure 4.16 interestingly showed changes which were on the scale of the frequency of applied electric field. The amplitude changes for both gelling agents, agar \& gelatin were observed only during application of electric fields. No obvious trend 
was observed in the amplitude changes either across different phantoms or across different windows of the same phantom. Observations from all such experiments lead to the fact that individual windows have

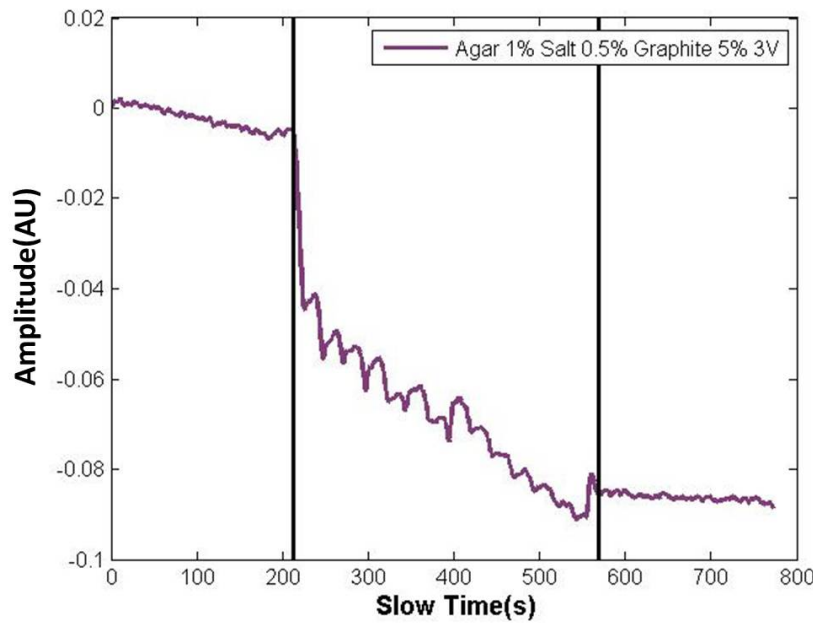

Figure 4.19: Amplitude changes near the front boundary of an agar $1 \%$ sample

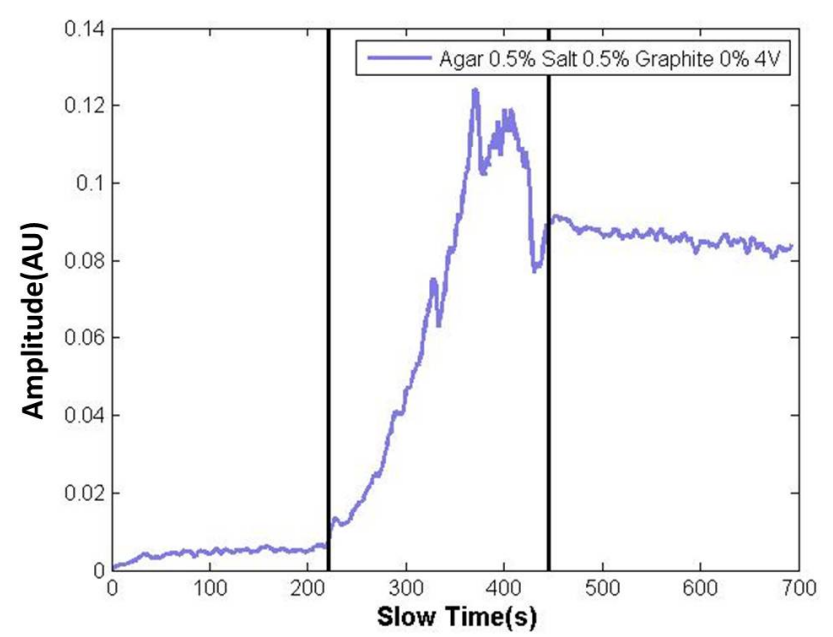

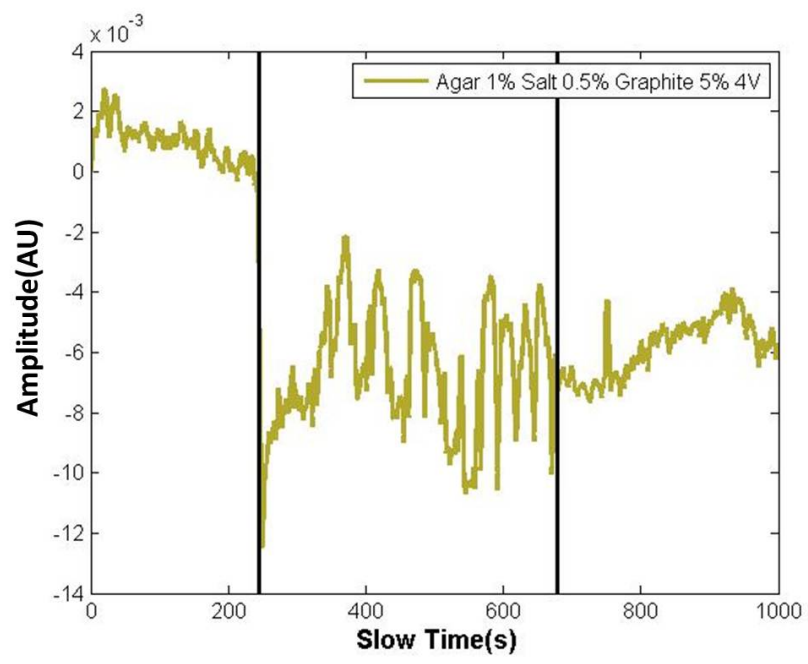

Figure 4.20: Amplitude changes near the front boundary of an agar $1 \%$ sample

Figure 4.21: Amplitude changes near the front boundary of an agar $0.5 \%$ sample

a different response to electric field application. The amplitude changes are highly variant throughout the experiments. 


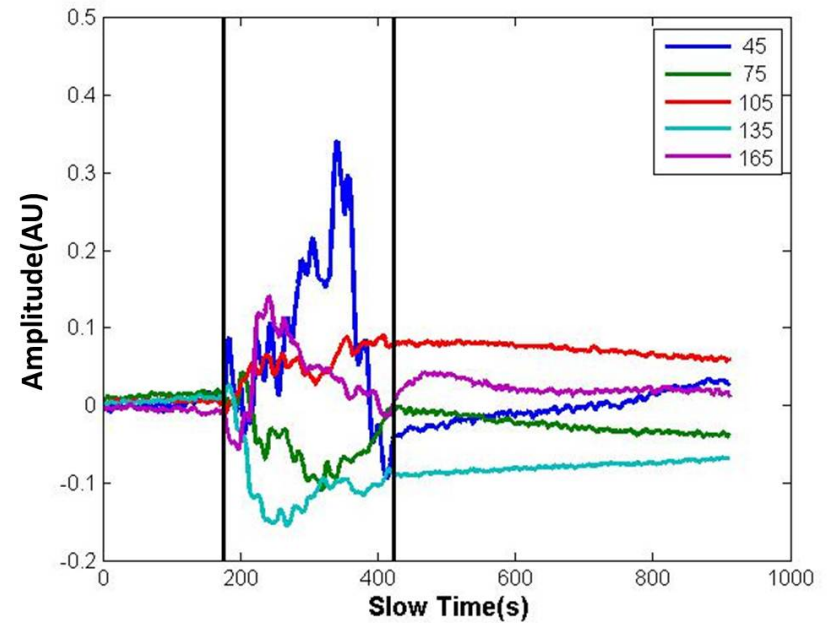

Figure 4.22: Amplitude changes within different windows of a Gel $6 \%$ sample

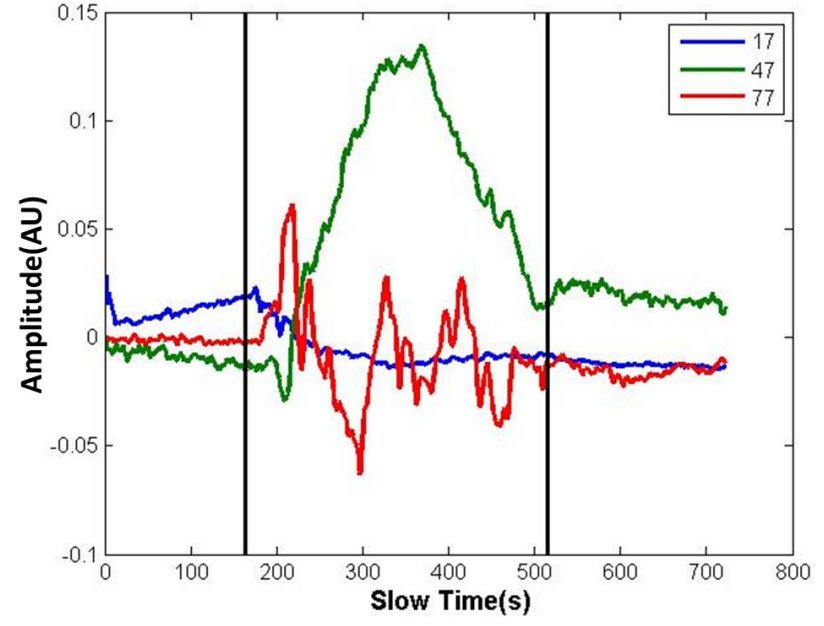

Figure 4.23: Amplitude changes within different windows of a Gel $9 \%$ sample

\subsection{Dependence of the EIMC on various experimental param- eters}

\subsubsection{Dependence on the applied electric fields}

\section{Dependence of the fluctuations within shifting of the RF signals}

The figure 4.24 displays the fluctuations as extracted from the shifting (as discussed in the Methods chapter - section 3.5.3). The triangular curves shown correspond to the direction of the applied electric field. The positive phase of the applied cycle would correspond to the increasing phase of the triangular curves and similarly the negative phase corresponds to the decreasing phase.

\section{Gel $3 \%$ and $6 \%$ phantoms}

As the frequency of the applied electric field is kept constant, data was analysed to observe the responses in shifting at the applied frequency of electric field. As described in the methods section, gelatin samples were compared for the fluctuations which correspond to the applied frequency. It was observed that the amplitude of fluctuations increased with the increase in electric field. Figure 4.26 and 4.28 show that for $3 \%$ and $6 \%$ gel samples, linear regression explained the trend very well.

\section{Gel 9\% phantoms}

Figure $4.29 \& 4.30$ show that the fluctuations present in $9 \%$ gels bore an exponential increase against the applied electric fields. This exponential trend was obtained only in the $9 \%$ gels. 


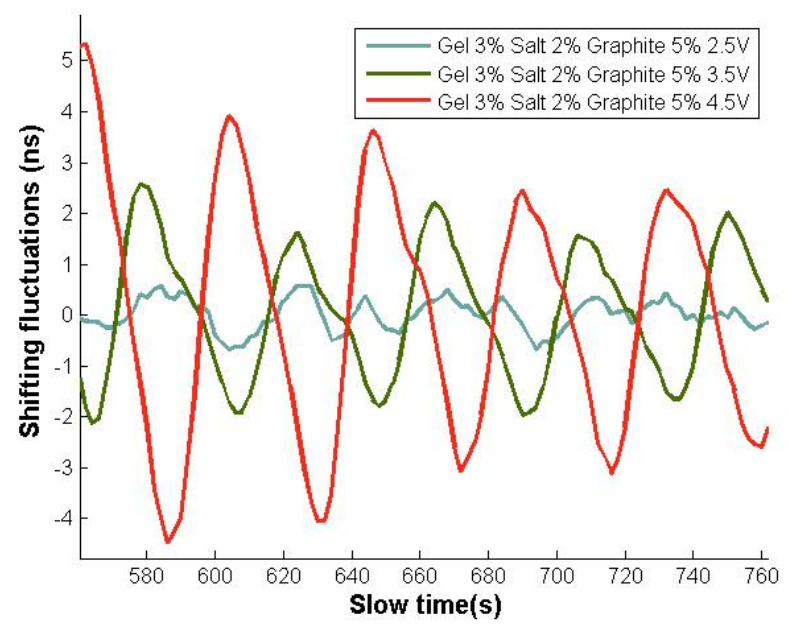

Figure 4.24: The shifting fluctuations in a $3 \%$ gelatin sample during the application of electric field.

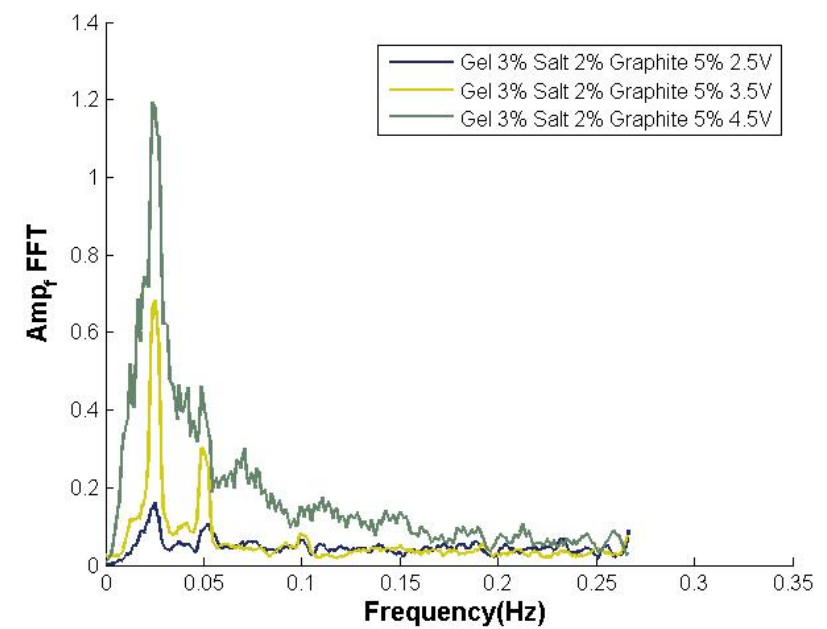

Figure 4.25: Frequency spectrum of the changes occurring during the application of electric field to a $3 \%$ gel sample.

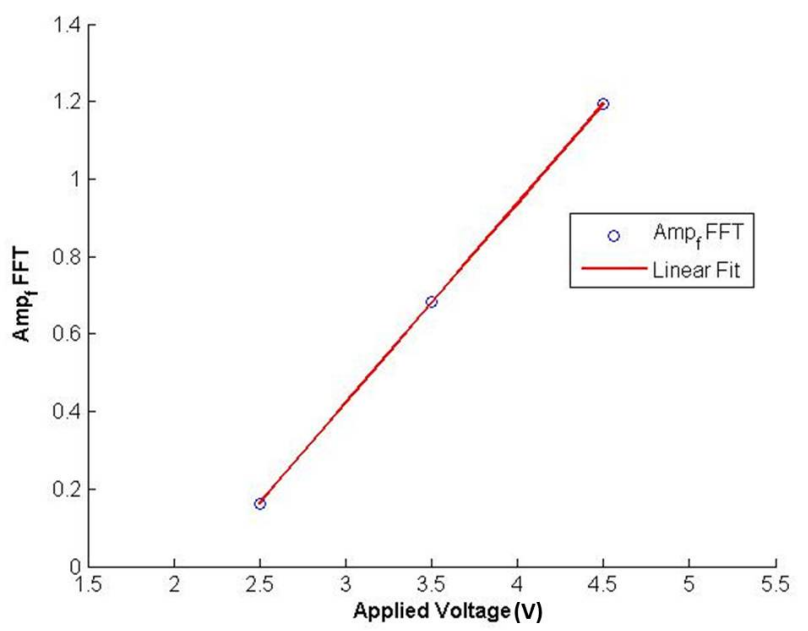

Figure 4.26: Linear regression performed on the amplitude of fft with respect to applied voltage of a $3 \%$ gel sample. 


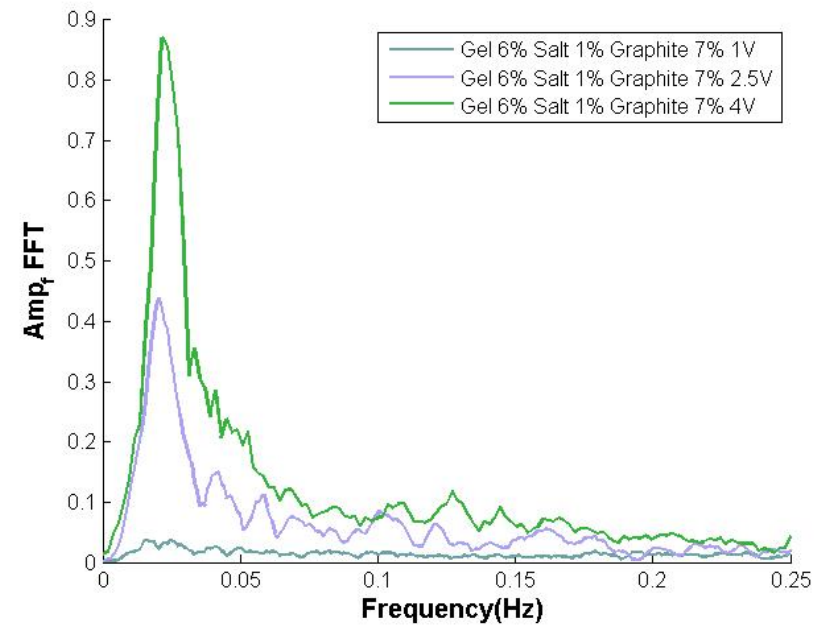

Figure 4.27: Frequency spectrum of the changes occurring during the application of electric field to a $6 \%$ gel sample.

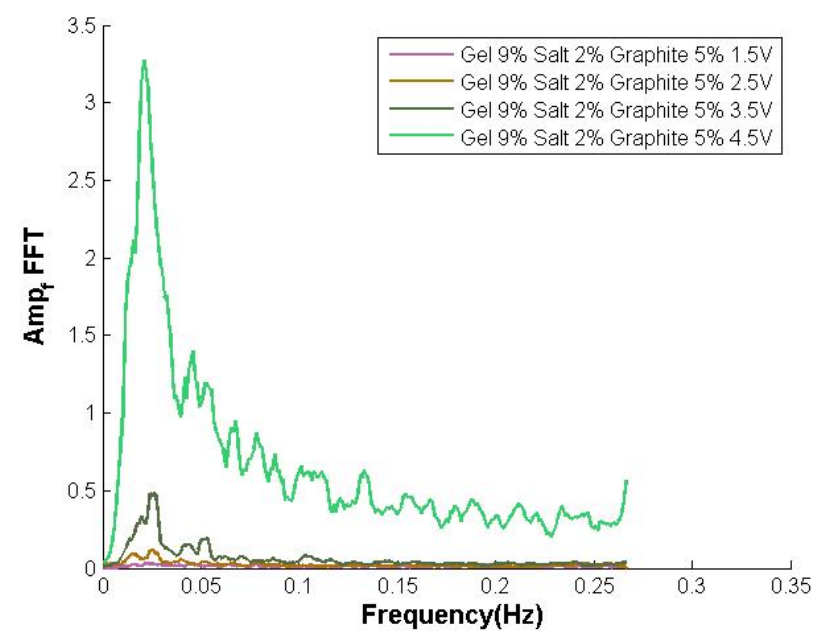

Figure 4.29: Frequency spectrum of the changes occurring during the application of electric field to a $9 \%$ gel sample.

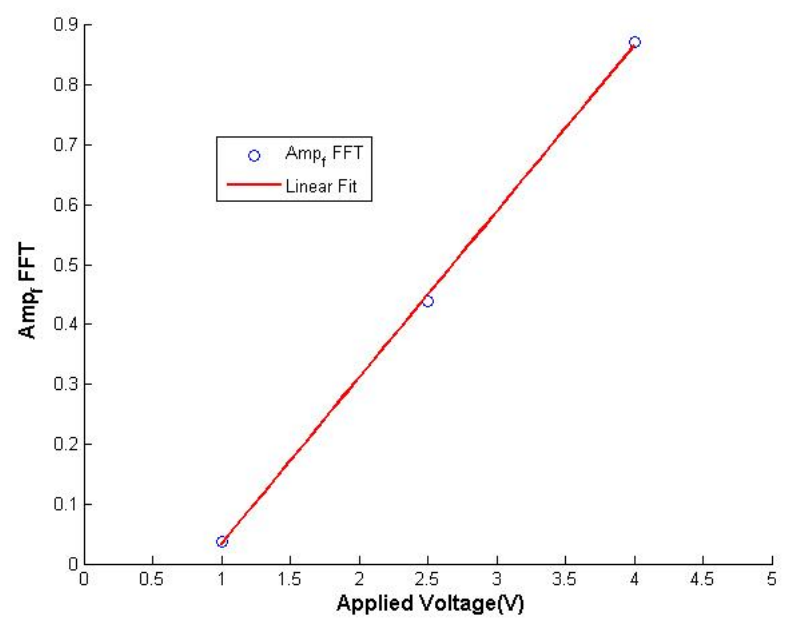

Figure 4.28: Linear regression performed on the amplitude of fft with respect to applied voltage $6 \%$ gel sample.

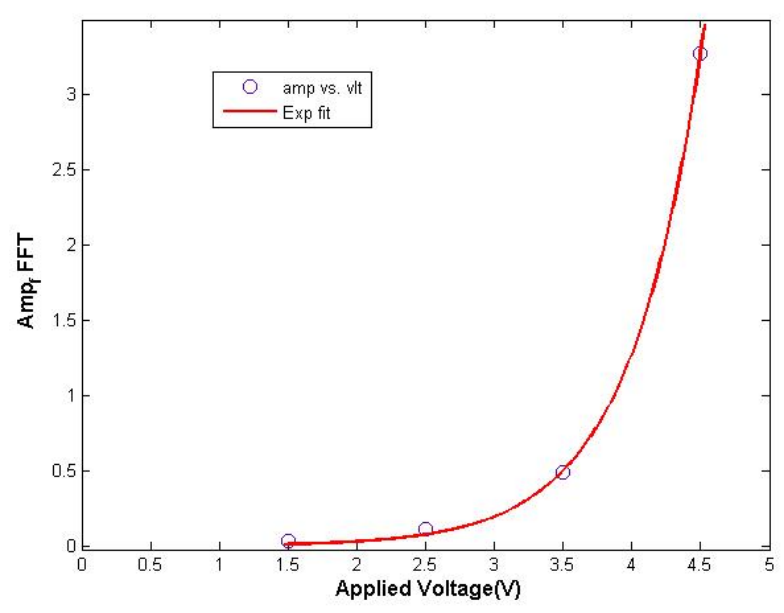

Figure 4.30: Linear regression performed on the amplitude of fft with respect to applied voltage $9 \%$ gel sample. 


\section{Dependence of the slow-time rate of change of shifting}

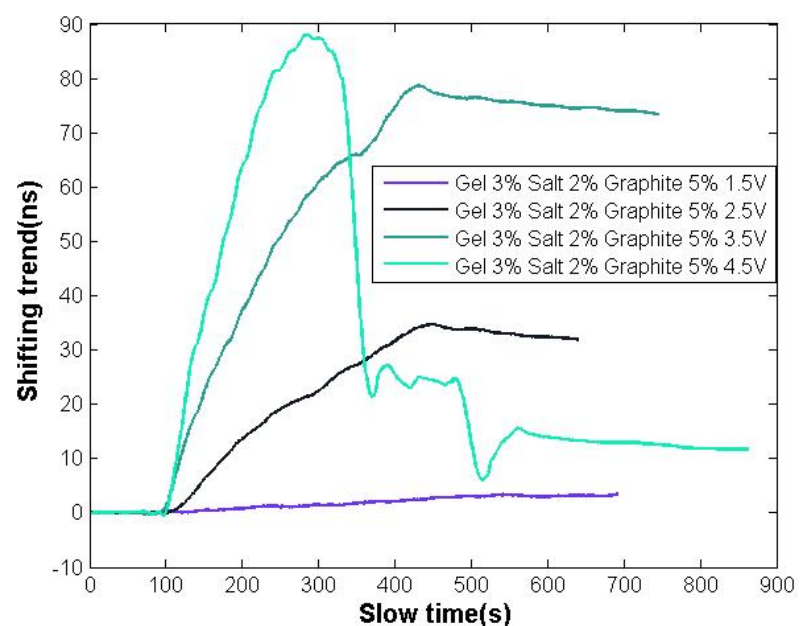

Figure 4.31: Slow-time shifting trends for various $3 \%$ gelatin samples

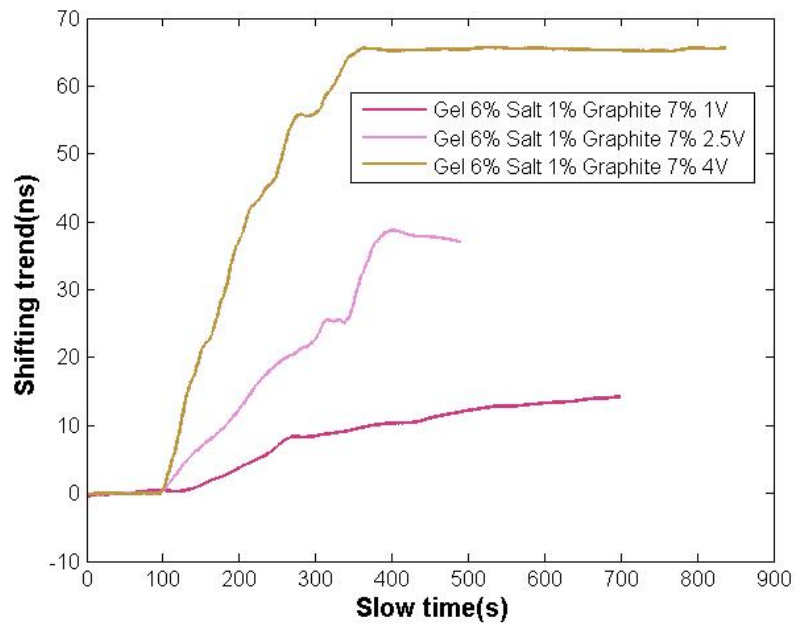

Figure 4.33: Slow-time shifting trends for various $6 \%$ gelatin samples

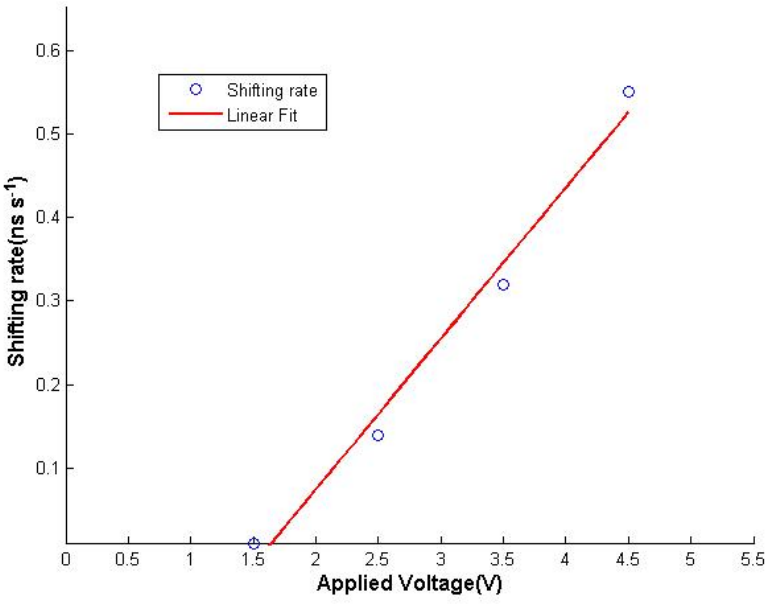

Figure 4.32: Shifting rate comparisons of 3\% gelatin samples to the applied voltage.

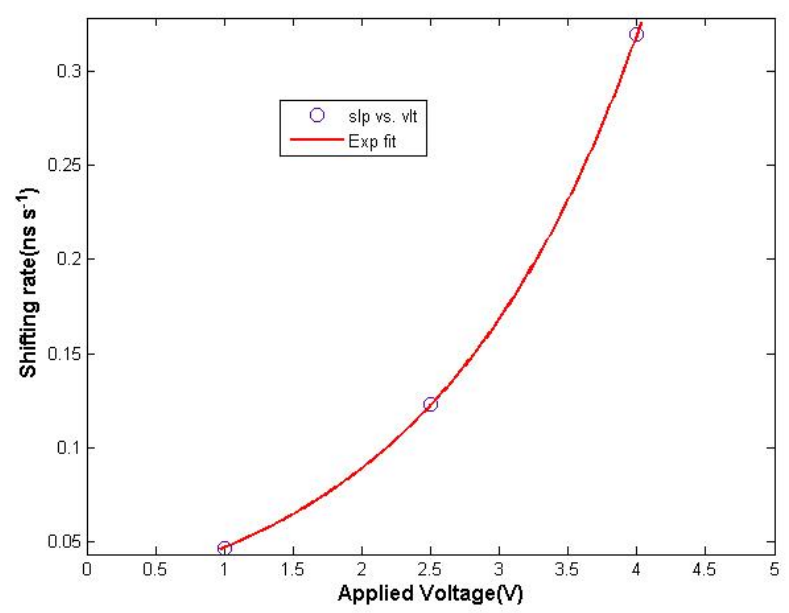

Figure 4.34: Shifting rate comparisons of $6 \%$ gelatin samples to the applied voltage.

Trends observed in the slow-time shifting for gel 3\% samples are shown in figure 4.31.Fig. 4.32 shows the slope of the shifting at the beginning of applying electric field (shifting rate) versus the applied voltage. As previously noted in shifting fluctuations, there was a linear trend in the shifting rate. The sample where $4.5 \mathrm{~V}$ application occurred, displayed a breakdown of shifting at approximately 300s. This might be attributed to some structural changes due to the strain developed by the application of electric field. Nonetheless, the trend observed is in accordance to the previous trends. It can be observed that 
the analysis of the shifting rate shows linear and exponential trends for all the phantoms. This rate of expansion of the phantoms was proportional to the applied electric fields. Figure $4.33 \& 4.34$ display the rates in $6 \%$ gelatin samples. An exponential increase in the rate was observed with respect to the applied voltage. Figure 4.35 shows a breakdown at a higher electric field for gelatin samples. It indicates that electric field might be inducing structural changes and at higher electric field strengths the non-linearity of the response is higher. A linear trend was observed for $3 \%$ gelatin phantoms whereas for $6 \%$ and $9 \%$ gel phantoms, an exponential trend explained the response well. It was also observed that at higher voltages, a breakdown was noticed. At even higher voltages (not displayed in the figures), non-linearity increased.

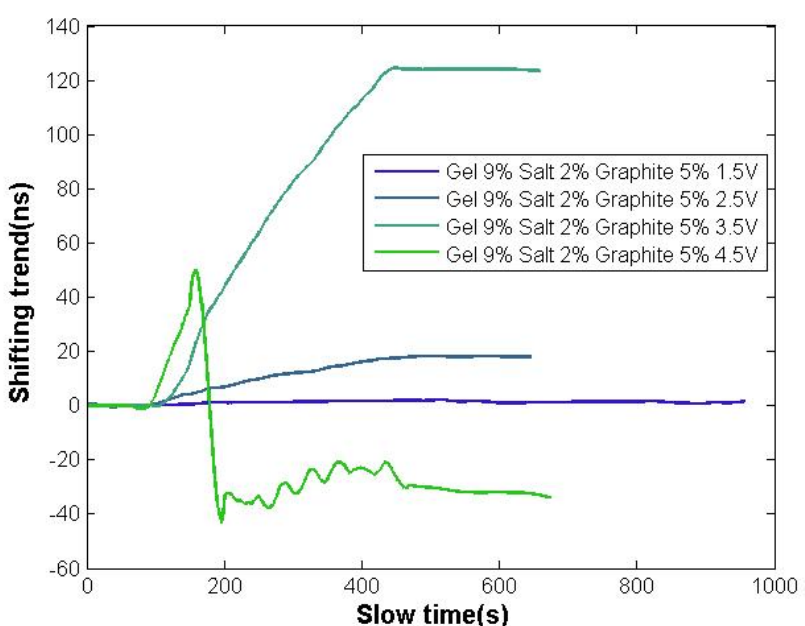

Figure 4.35: Slow-time shifting trends for various $9 \%$ gelatin samples

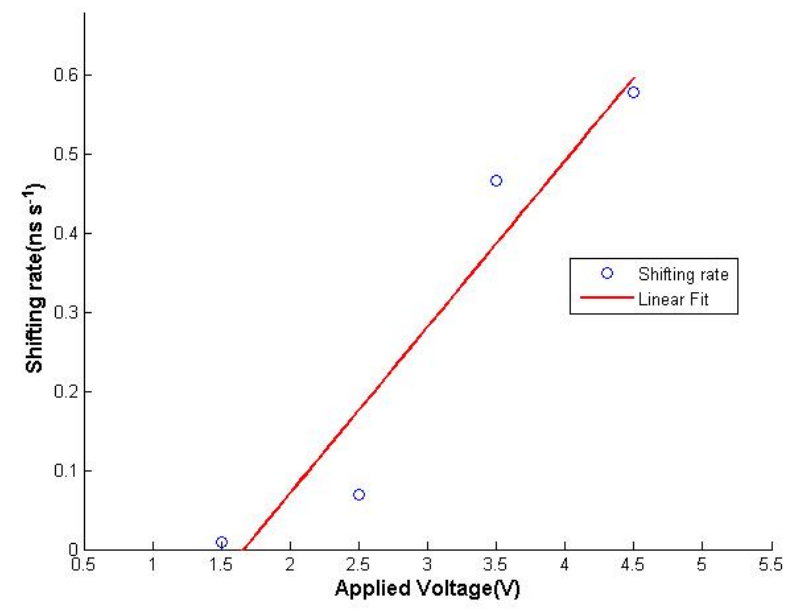

Figure 4.36: Shifting rate comparisons of $9 \%$ gelatin samples to the applied voltage. 


\subsubsection{Dependence on the concentration of gelling agents}

\section{Variability in results}

The results obtained from all the experiments at different parameters show a high variability. Figure 4.37 $\& 4.38$ show the variability in strain for gelatin and agar experiments. The value of strain is normalised by the applied electric field. The blue rectangles in both the figures indicate the response right after the onset of application. The area displayed by the blue area for gelatin experiments seem to correspond to a linear trend. The area denoted by the red rectangle displays that the response becomes nonlinear as the time increased. Prolonged exposure of the phantom to electric fields ultimately would result in structural changes leading to an erratic response. The results were mutually compared within the blue and black boxes as they displayed a linear response to the applied electric fields.

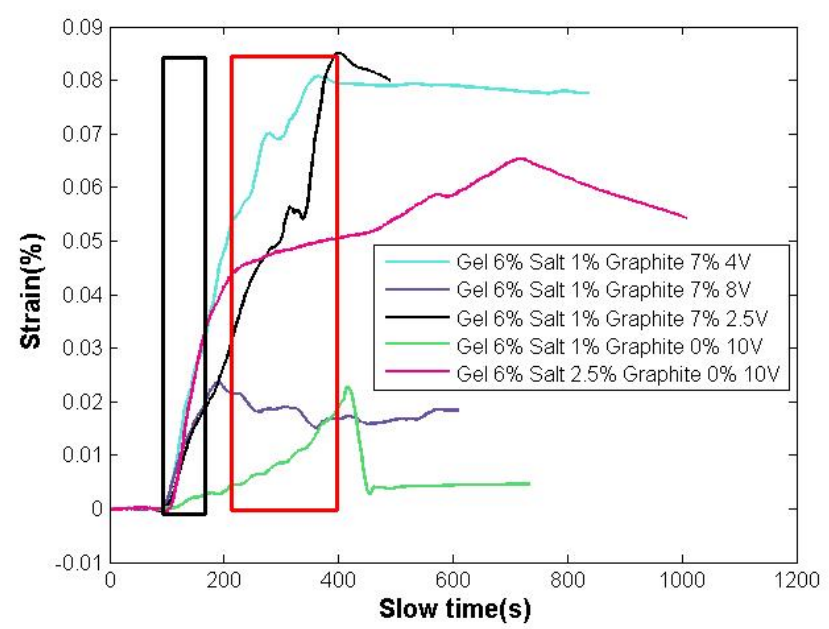

Figure 4.37: Variability in $6 \%$ gel experiments

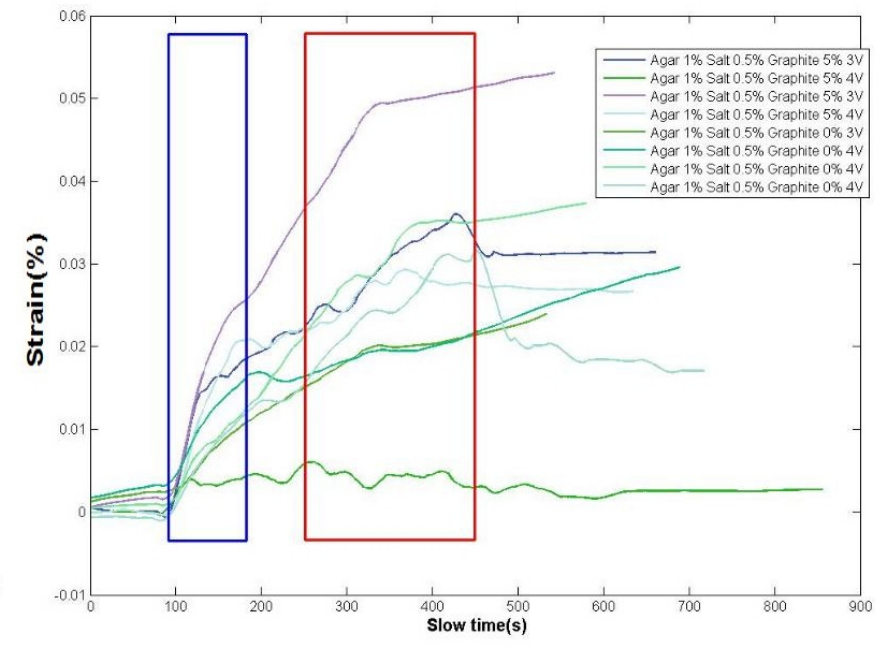

Figure 4.38: Variability in 1\% agar experiments

\section{Comparison of gelatin and agar experiments}

Changes in gelatin concentration of samples affects the response of the phantom. Figure 4.39 displays strain in multiple phantoms. The strain plots are grouped together based on the concentration of gelatin used. Figure 4.42 compares four different experiments with different gelatin concentrations. The rate of change of strain increased with an increase in the gelatin concentration when the concentration was $<$ $9 \%$. Then the strain rate decreased with the increasing gelatin concentration. Fig. 4.434 .44 displays the strain profile of agar phantoms. The profiles follow a different trend when compared to gelatin phantoms. Higher concentration of agar portrayed a lower strain profile. Agar gels are much different than gelatin gels. In order to form firm gels, a very low concentration is required when compared to gelatin gels. Expansions within the phantoms were measured approximately from $30 \mu m$ to $150 \mu m$ and the strain generated in various samples ranged from $0.15 \%$ to $0.8 \%$. 


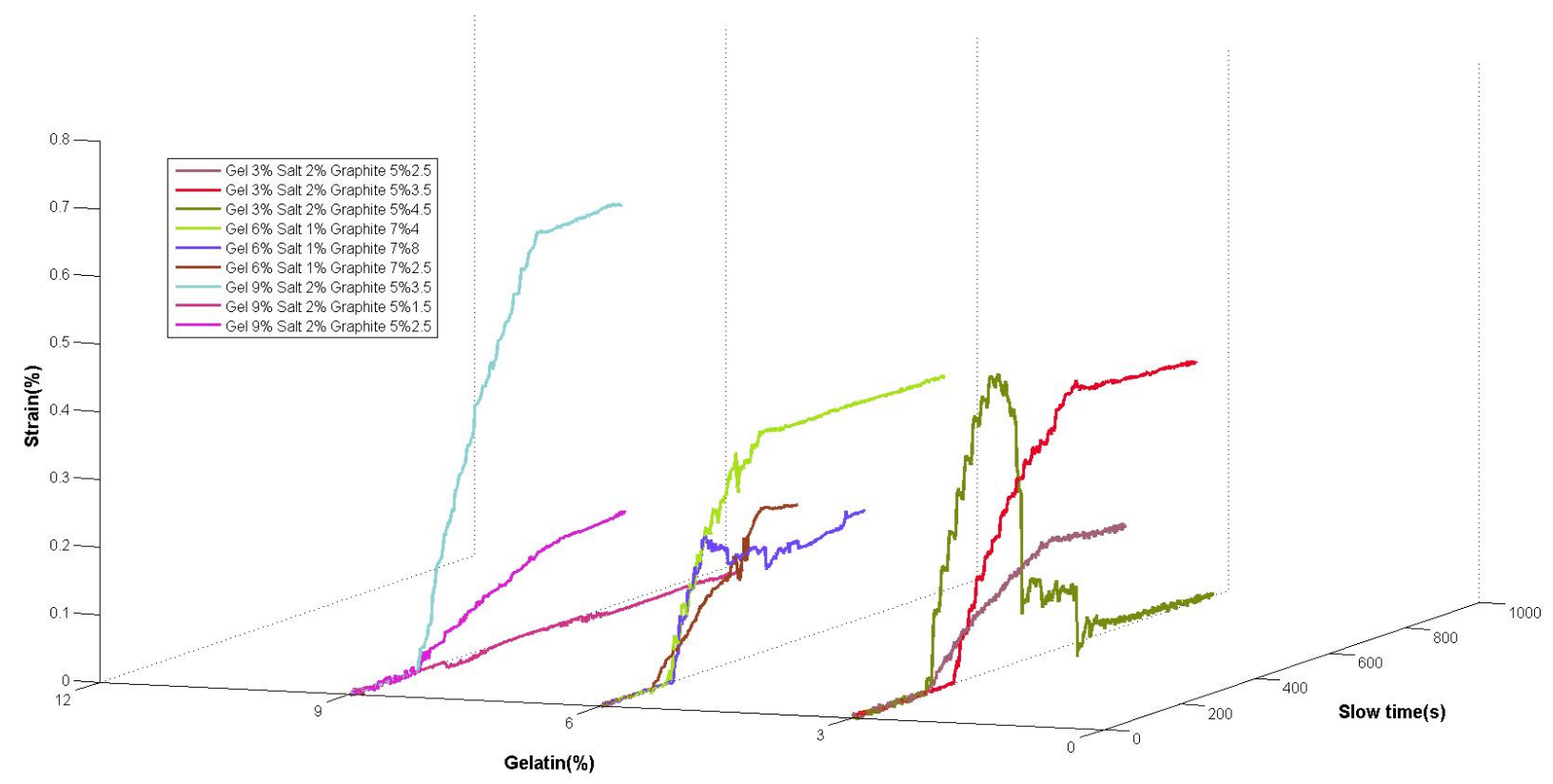

Figure 4.39: Comparision of strain profiles generated for different strengths of gelatin

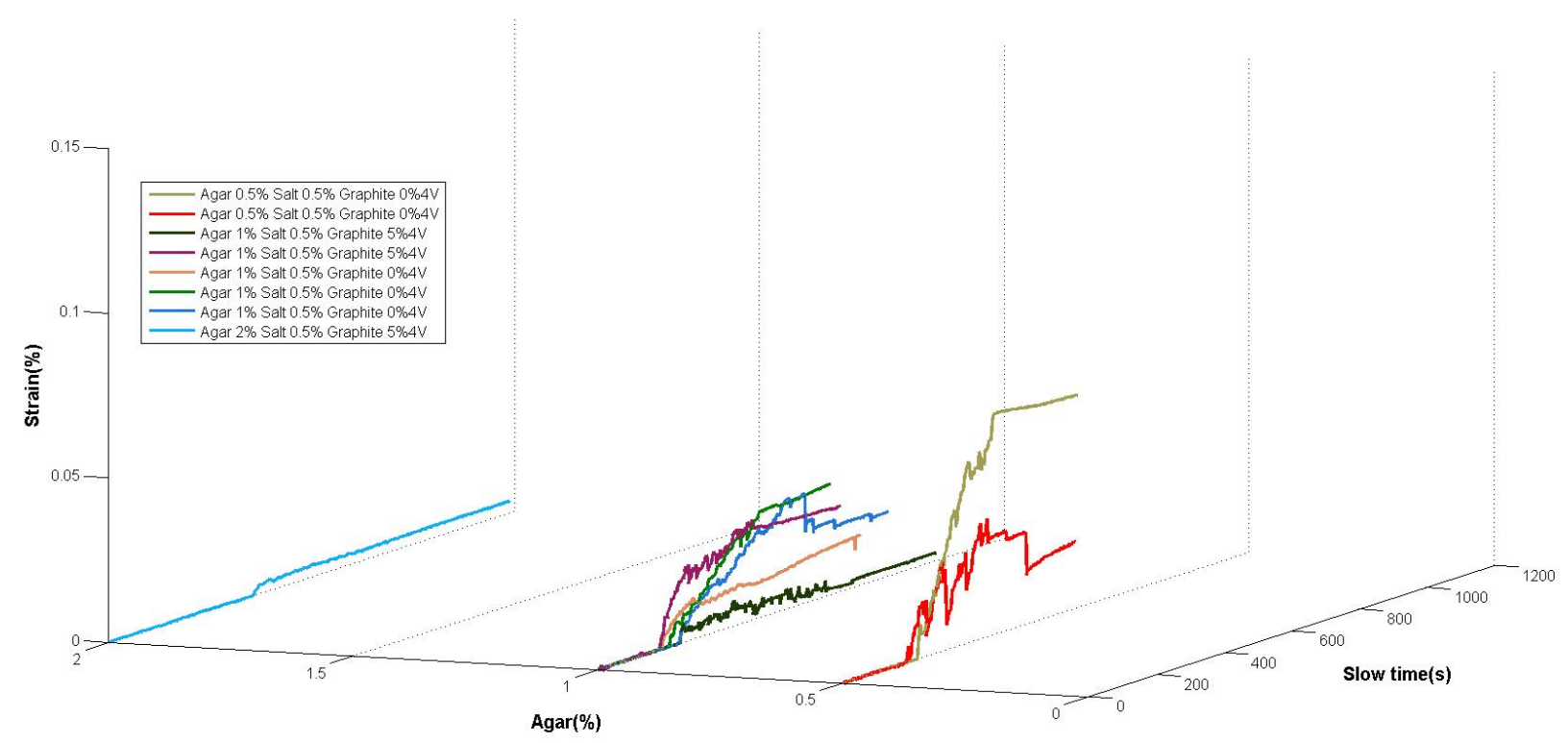

Figure 4.40: Comparision of strain generated for different strengths of agar 


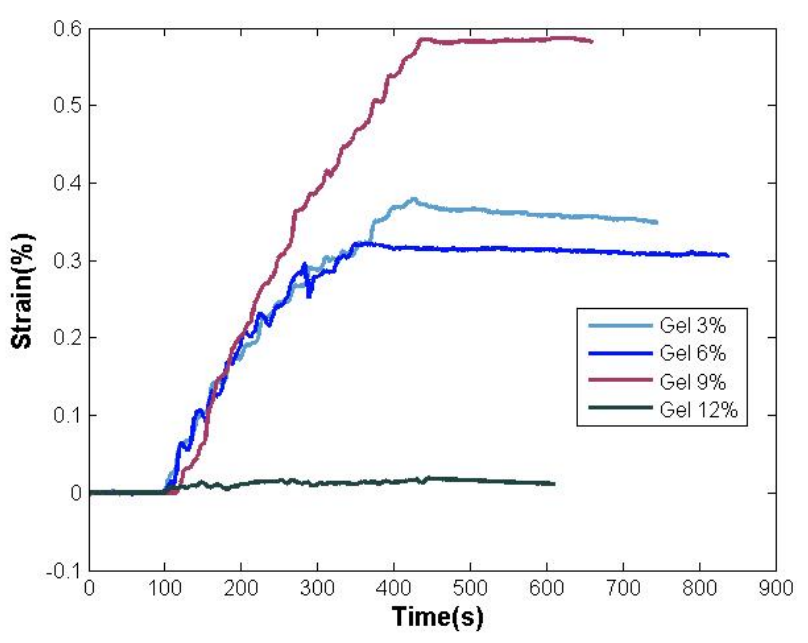

Figure 4.41: Slow time strain comparison for gelatin phantoms with varying gelatin concentrations at the same experimental conditions

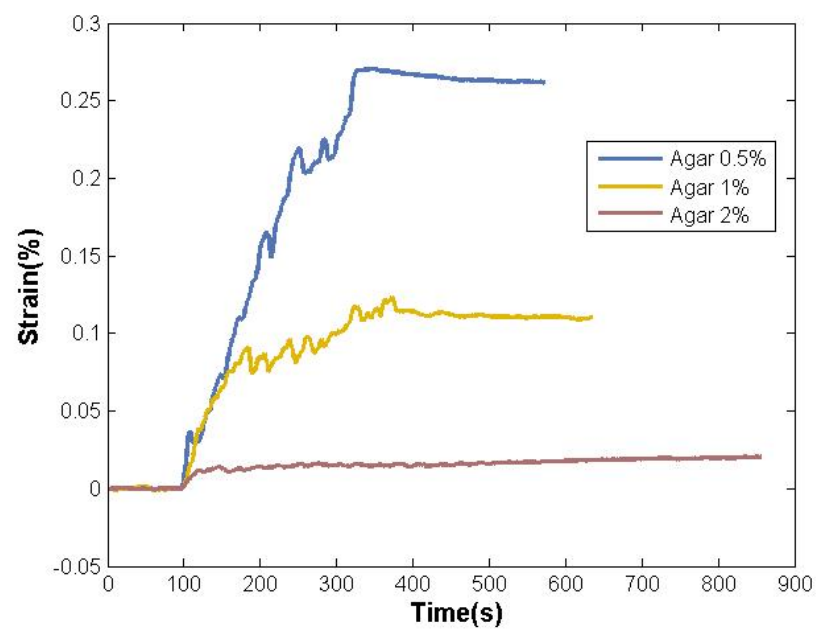

Figure 4.43: Slow time strain comparison for gelatin phantoms with varying agar concentrations at the same experimental conditions

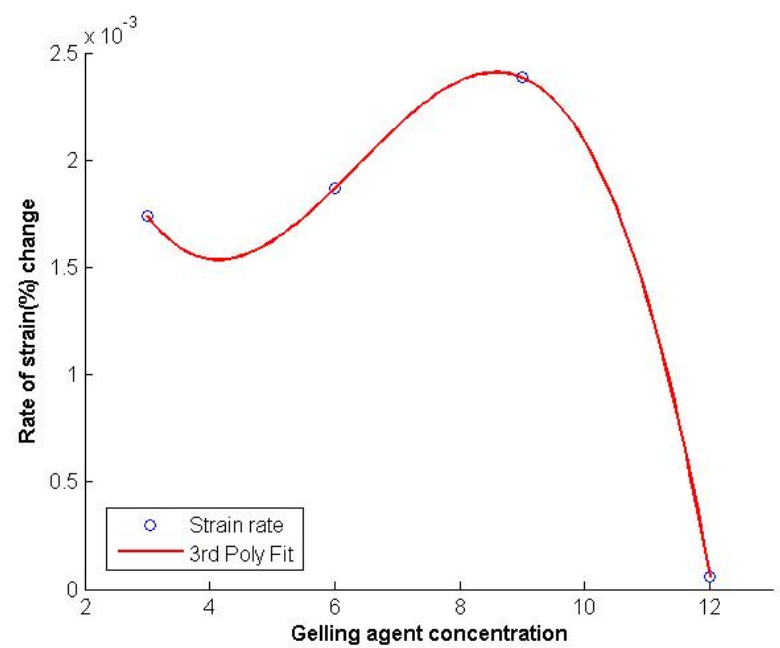

Figure 4.42: The dependence of the rate of change of strain on the concentration of gelatin.

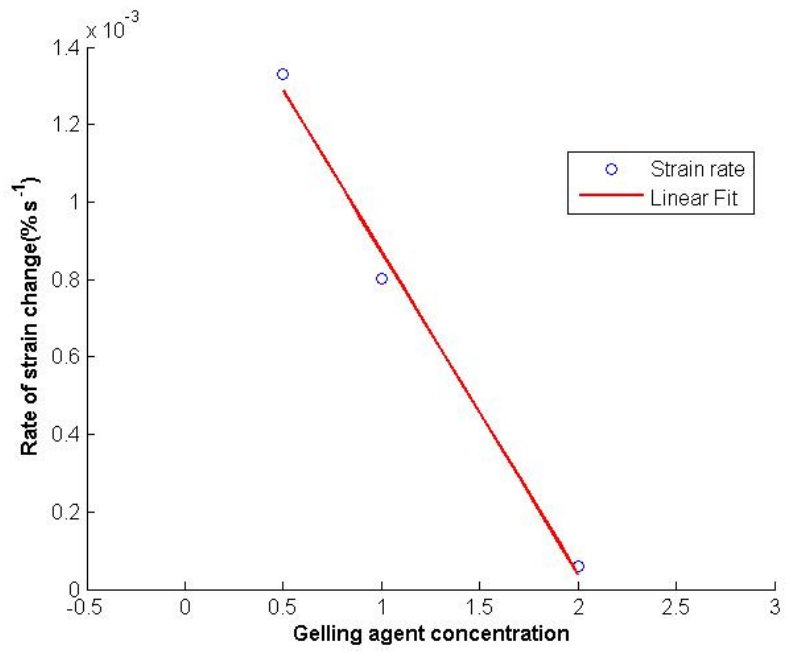

Figure 4.44: The dependence of the rate of change of strain on the concentration of agar. 


\subsubsection{Dependence on salt concentration}

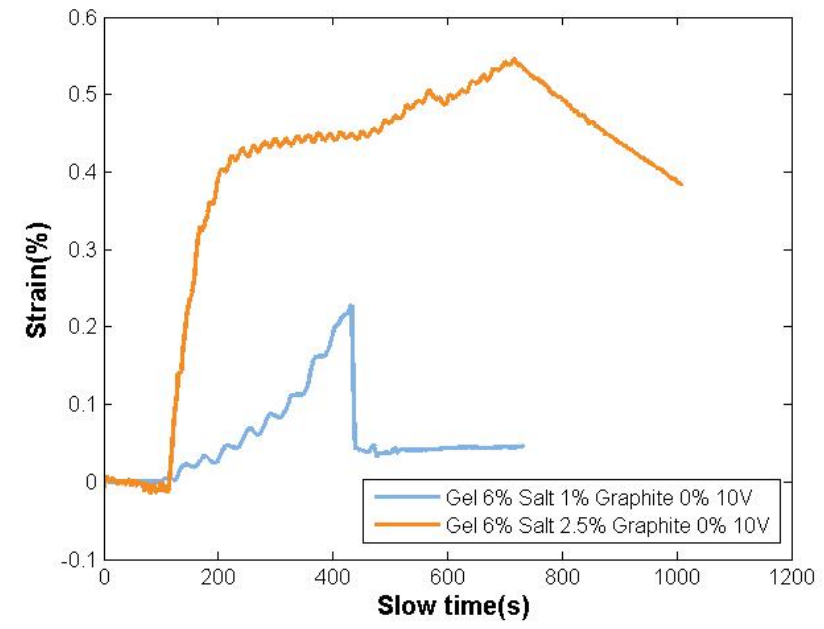

Figure 4.45: Effect of increasing salt concentration in $6 \%$ gelatin phantoms

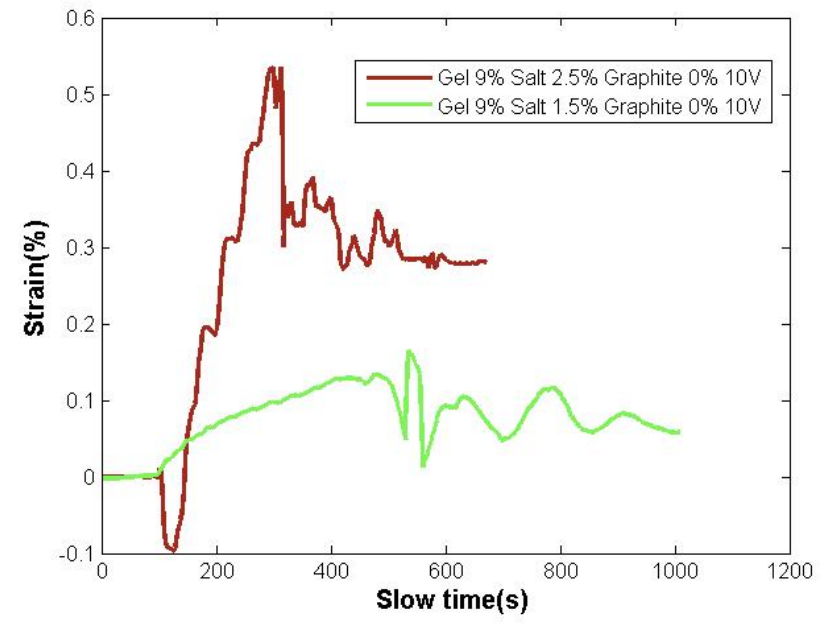

Figure 4.46: Effect of increasing salt concentration in $9 \%$ gelatin phantoms

Increasing concentration of salt can have the same effect as increasing the temperature. The gel softness decreases as the concentration of salt increases [38. Consequently, the magnitude of EIMC increases with the increasing salt concentration of the sample $4.45-4.46$. The conductivity increases with the salt concentration while the stiffness decreases.

\subsubsection{Comment on temperature changes during the experimental procedure}

Since the experiments involved a flow of electric current, joule heating of the phantom had to be considered. Temperature was recorded at three locations within the phantom; close to the two injecting electrodes and one at the center of the phantom. Both AC and DC electric fields were applied and temperature changes were recorded from these three locations. Table 4.2 shows the temperature records. It was observed that the temperature changes, occurring during the application of electric fields, were not significant. The changes in temperature were around $0.1-0.2{ }^{\circ} \mathrm{C}$. The changes in ultrasound signals due to such a temperature change would be negligible compared to the strain generated by the electric field. 
Table 4.2: Temperature measurements in Phantoms

\begin{tabular}{|l|c|c|c|c|}
\hline Type of Phantom & Room Temperature ${ }^{\circ} \mathrm{C}$ & Left Electrode ${ }^{\circ} \mathrm{C}$ & Center $^{\circ} \mathrm{C}$ & Right Electrode ${ }^{\circ} \mathrm{C}$ \\
\hline $\begin{array}{l}\text { Gel } 6 \% \text { no salt no } \\
\text { scatterers before } \vec{E}\end{array}$ & 22.7 & 21.7 & 22.0 & 22.0 \\
\hline $\begin{array}{l}\text { Gel } 6 \% \text { no salt no } \\
\text { scatterers after AC } \\
\vec{E}\end{array}$ & 22.7 & 21.5 & 21.8 & 21.9 \\
\hline $\begin{array}{l}\text { Gel } 6 \% \text { no salt no } \\
\text { scatterers after DC } \\
\vec{E}\end{array}$ & 22.7 & & & \\
\hline $\begin{array}{l}\text { Gel } 6 \% 0.5 \% \text { salt } \\
\text { no scatterers before }\end{array}$ & & 21.6 & 21.7 & 21.7 \\
$\vec{E}$ & 22.2 & & & \\
\hline $\begin{array}{l}\text { Gel } 6 \% 0.5 \% \text { salt } \\
\text { no scatterers after }\end{array}$ & 22.2 & 21.6 & 21.7 & \\
AC $\vec{E}$ & & & & 21.8 \\
\hline $\begin{array}{l}\text { Gel } 6 \% 0.5 \% \text { salt } \\
\text { no scatterers after }\end{array}$ & 22.2 & 21.7 & 21.7 & \\
DC $\vec{E}$ & & & & \\
\hline
\end{tabular}

\subsection{Experiments with a linear array transducer to generate im- ages based on EK effects.}

B-mode images were obtained based on the experimental protocol explained in the methods section. An image was created using time delay estimates generated using the cross correlation algorithm. To better understand changes in the signals, an image formed by using a color map showing color coded values of TDE was overlaid over a gray-scale B-mode image. Figure 4.47 displays such an overlay where images $a, b, c, d$ were obtained using overlays from TDE images and images corresponding to the changes in amplitude. The tissue under examination was a porcine cardiac tissue which was extracted from the ventricular wall of a porcine heart. Part $e$ of the figure portrays a normal grayscale B-mode image of the tissue under examination. $a$ and $b$ are the overlays formed before application of electric field to the tissues. As noted in the figure, the changes observed before application of electric fields were insignificant uniformly throughout the tissue structure. When exogenic fields to the tissues were applied, local changes in time delay estimates were observed within the tissue structure. These changes implied internal motion of the tissue structure. In part $c$, the area corresponding to the sampling points $100-300$ and the A-lines $50-220$ showed an increase in the time delay suggesting the expansion of tissues within that region. 
a

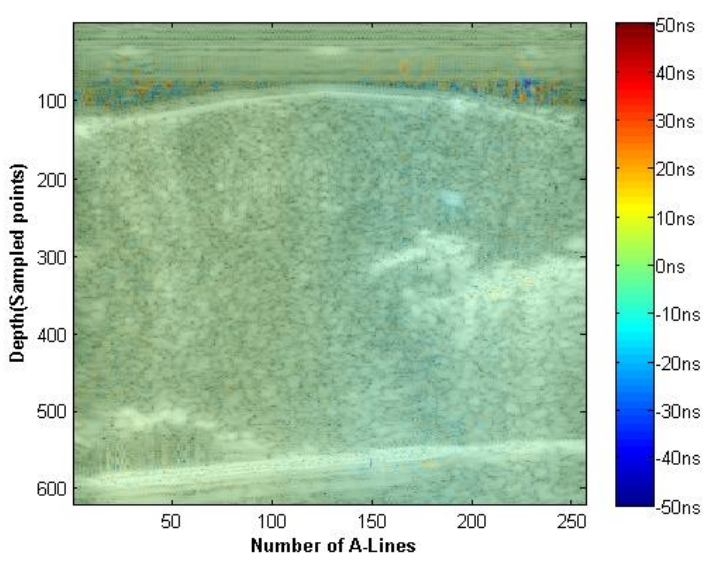

C

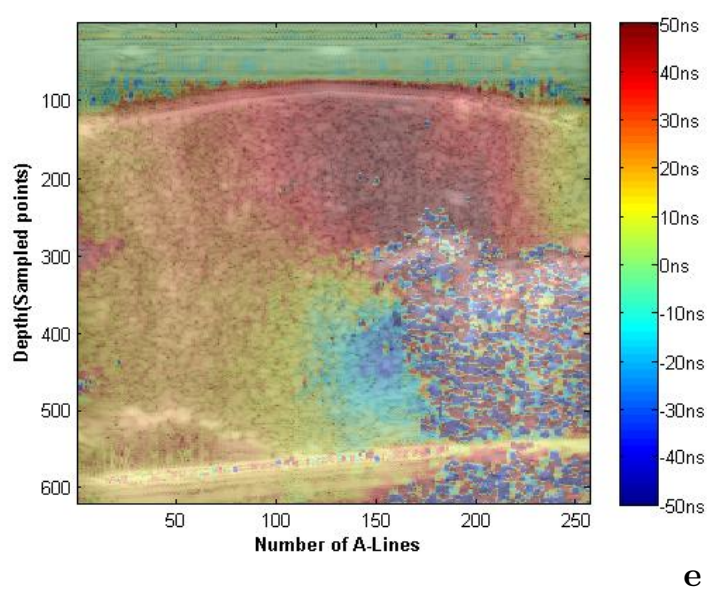

b

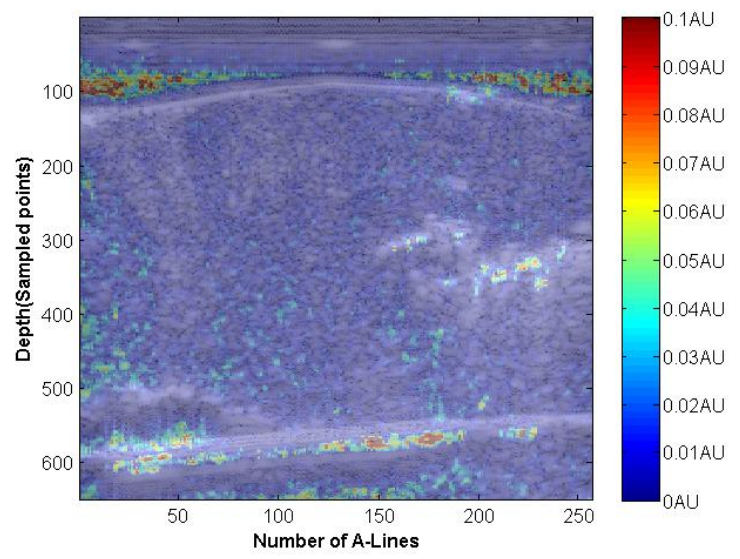

d

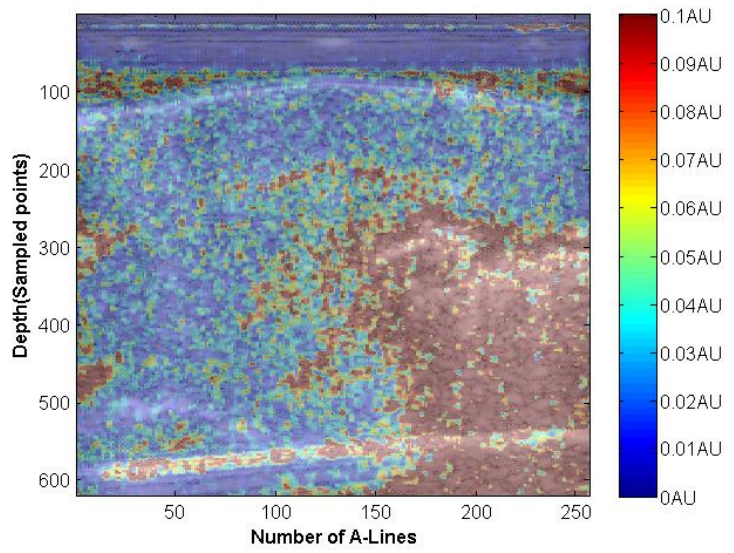

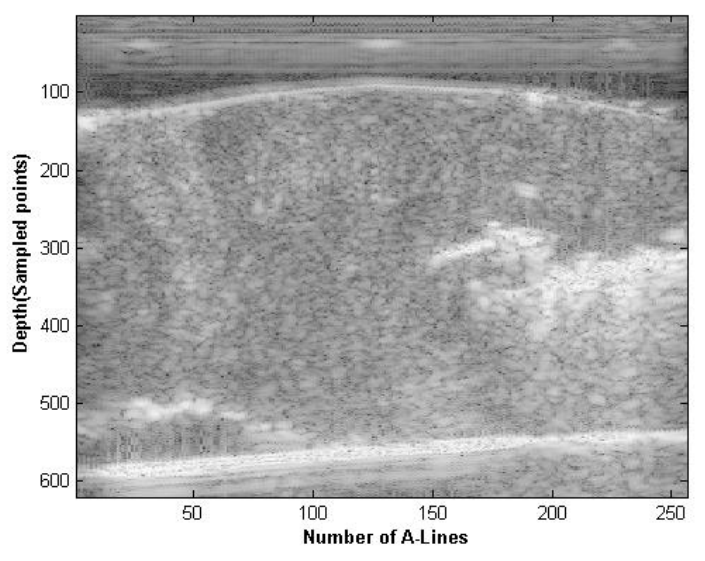

Figure 4.47: Porcine cardiac tissue - Sample I: (a) B-Mode image overlaid with an RGB image generated by the magnitude of the Localised Time Delay Estimates(TDE) before the application of $\vec{E}$ field . (b) B-Mode image overlaid with an RGB image generated by the magnitude of the Localised Amplitude Changes(LAC) before the application of $\vec{E}$ field. (c) TDE overlay after the application of $\vec{E}$ field. (d) LAC overlay after the application of $\vec{E}$ field. (e) B-Mode image of the sample under examination. 
Another tissue sample, a two layered structure of fat and muscle, was extracted from porcine stomach. Figure 4.48 e shows the B-mode image of such a tissue structure. The same analysis was performed on this sample and the results were displayed in part $c$ and part $d$ of the figure. It was found that the layer, which represented fat tissue, showed a lower response when compared to the layer of muscle tissue. The layers of fat and muscle can be distinguished in part $c \& d$ which display the TDE and amplitude changes respectively. The sample shows approximately $2.5 \mathrm{~cm}$ in depth.

A different cardiac tissue sample was obtained and analysed for electrical field induced changes. Figure 4.49 displays the results obtained from the experiment. Part $a \& c$ show the time delay estimates before and after the application of electric field respectively. $b$ and $d$ display the absolute amplitude changes. The application of electrc field induced mechanical changes within the tissue structure. These changes observed were throughout the sample at various locations. Part $d$ shows that the amplitude changes were specific to the area represented by a depth of $300-400$ sampling points and the A-lines $25-110$ approximately. The amplitude changes represent the change in the backscattering properties of the tissue sample.Figure 4.50 was used to display the changes in a muscle tissue. All the figures show an image artifact in the first fifty A-lines. This was due to the fact that the size of the sample was smaller in dimension than the size of linear array. The algorithm generated erratic results within these boundaries. The boundaries were distinct indicating the robustness of the algorithm.

Figure $4.51 \& 4.52$ show experiments performed on ultrasound phantoms bearing graphite as scatterers. The salt concentrations used for these phantoms was $1 \%$. The phantoms showed a response which was almost homogeneous. Figure 4.52 shows a phantom where a layer of graphite particles sedimented thus forming layers within the phantoms. It could be observed that the response was different for different layers of the phantom.

For the experiments performed on linear array transducers, the maps of the time delay estimates provided a contrast based on the changes in arrival time of the windowed ultrasound signal. The amplitude changes were also used to generate color coded images to observe spatial variations. It was seen that in some cases, the contrast provided by TDE images was able to distinguish between the layers of tissues (Fig 4.47). It was also seen that there were some contrasting features which displayed the local variations within the tissues (Fig. 4.48). An ultrasound phantom, with graphite as scatterers showed almost uniform variations throughout the structure indicating that phantoms can be used to characterise such effects(Fig 4.51). 
a

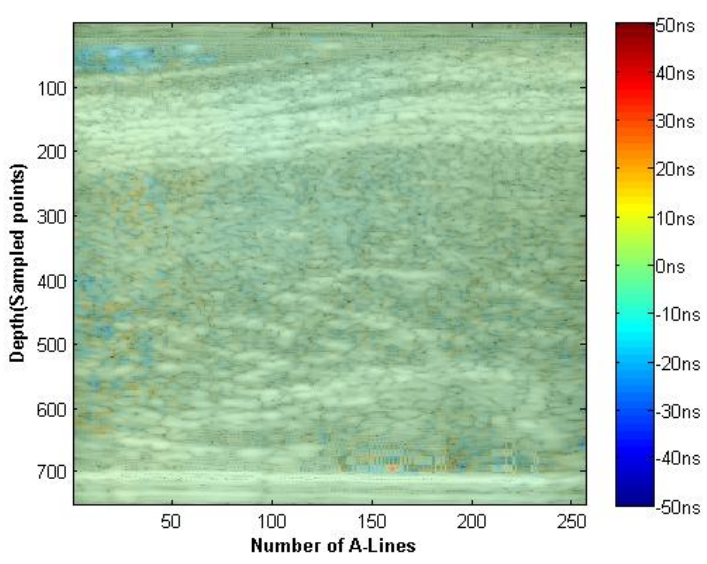

C

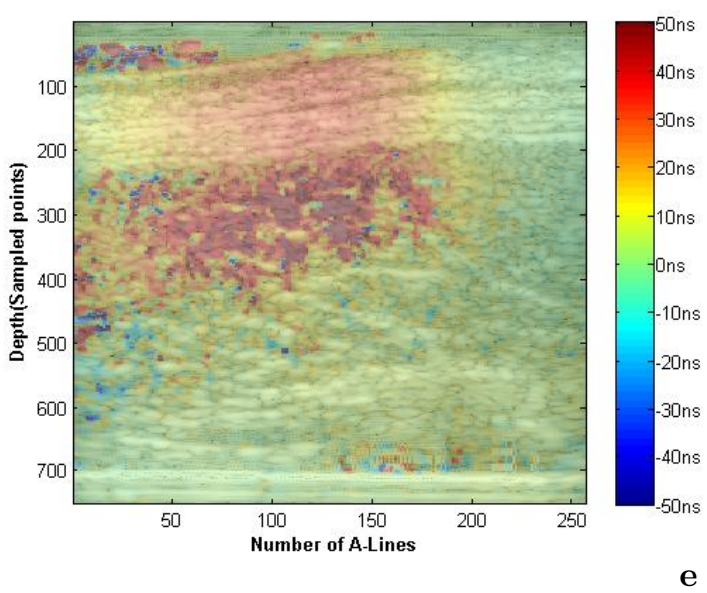

b

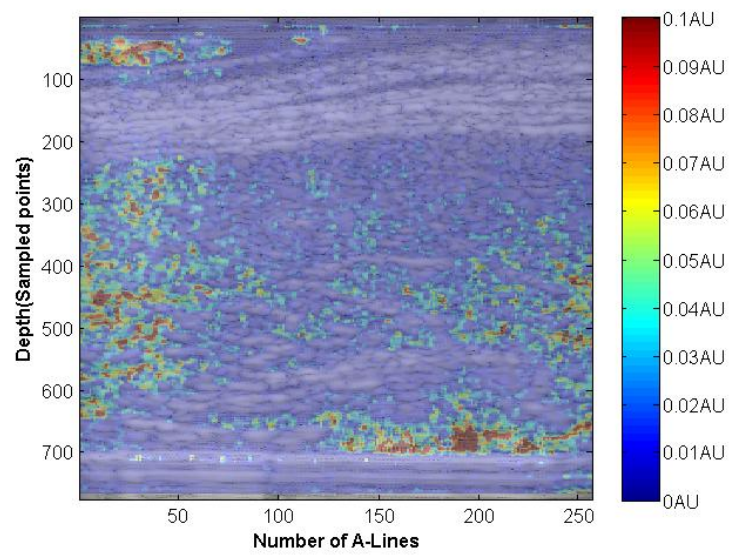

d

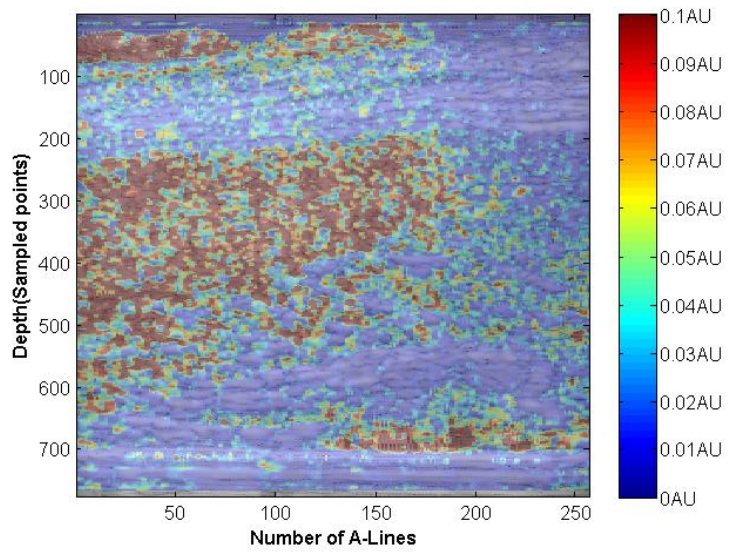

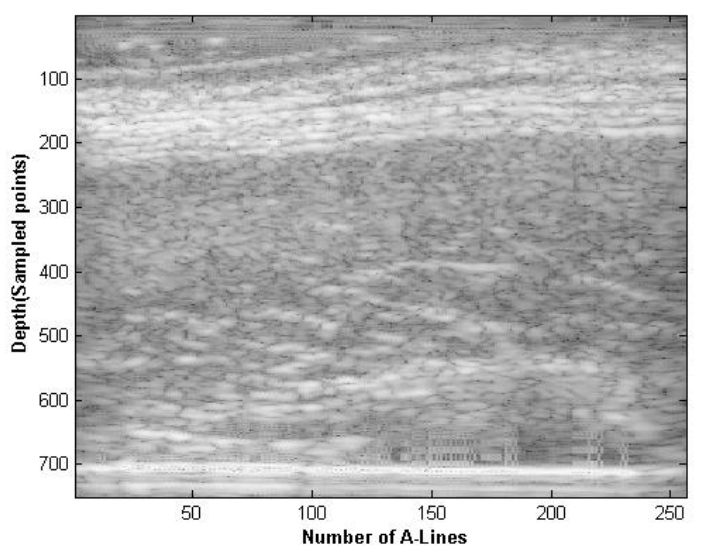

Figure 4.48: Porcine layered tissue(Muscle and Fat) : (a) B-Mode image overlaid with an RGB image generated by the magnitude of the Localised Time Delay Estimates(TDE) before the application of $\vec{E}$ field . (b) B-Mode image overlaid with an RGB image generated by the magnitude of the Localised Amplitude Changes(LAC) before the application of $\vec{E}$ field. (c) TDE overlay after the application of $\vec{E}$ field. (d) LAC overlay after the application of $\vec{E}$ field. (e) B-Mode image of the sample under examination. 
a

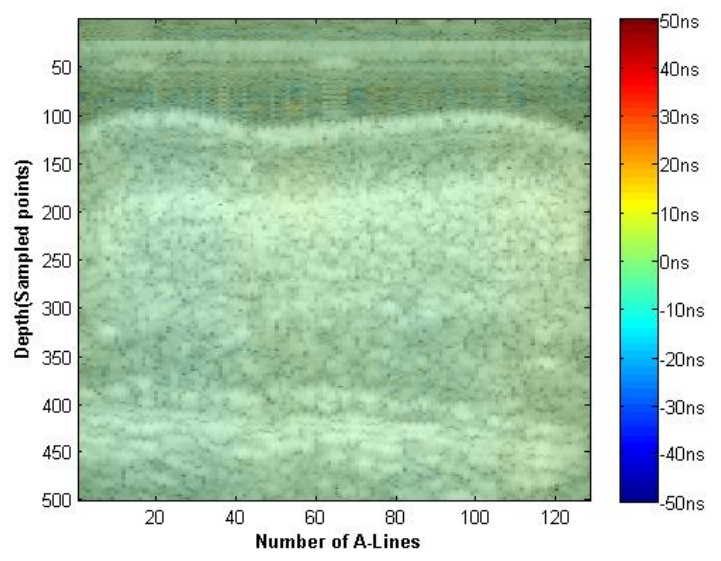

C

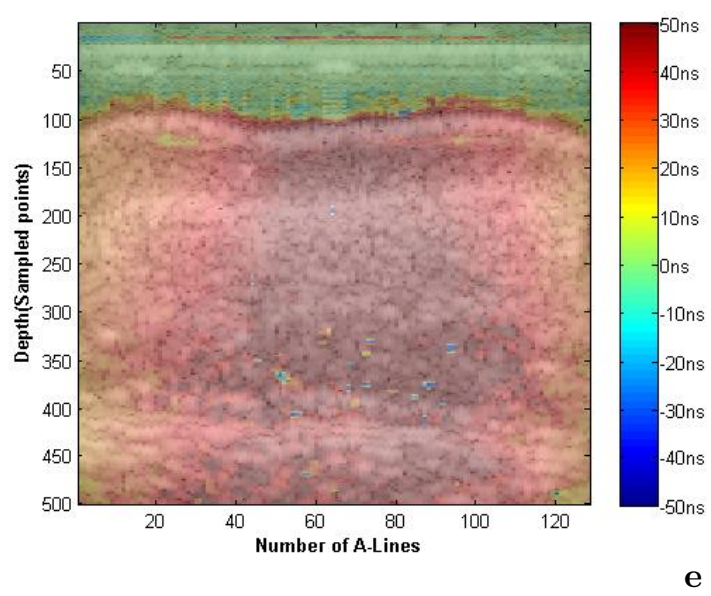

e b

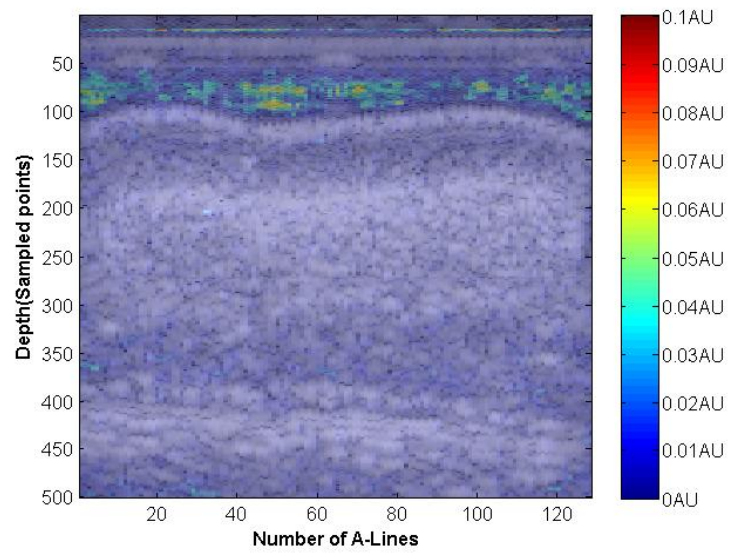

d

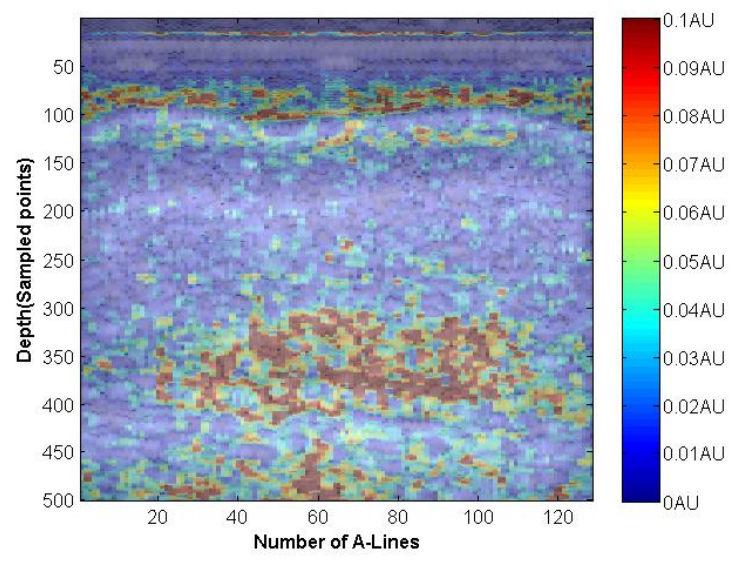

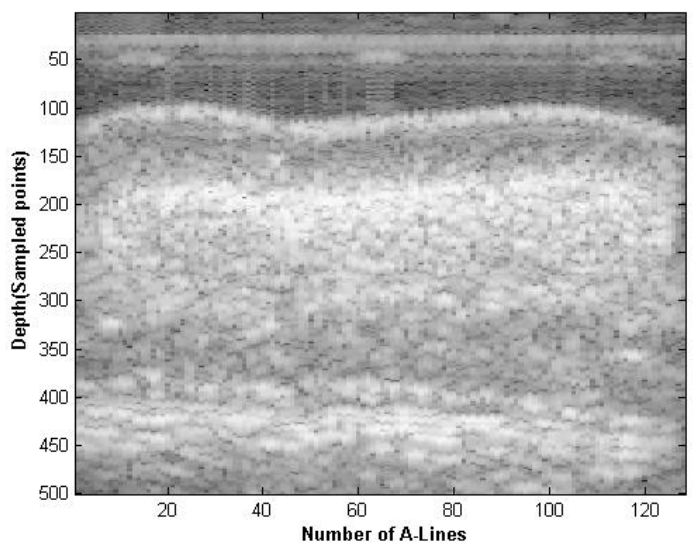

Figure 4.49: Porcine cardiac tissue - Sample II : (a) B-Mode image overlaid with an RGB image generated by the magnitude of the Localised Time Delay Estimates(TDE) before the application of $\vec{E}$ field . (b) B-Mode image overlaid with an RGB image generated by the magnitude of the Localised Amplitude Changes(LAC) before the application of $\vec{E}$ field. (c) TDE overlay after the application of $\vec{E}$ field. (d) LAC overlay after the application of $\vec{E}$ field. (e) B-Mode image of the sample under examination. 
a

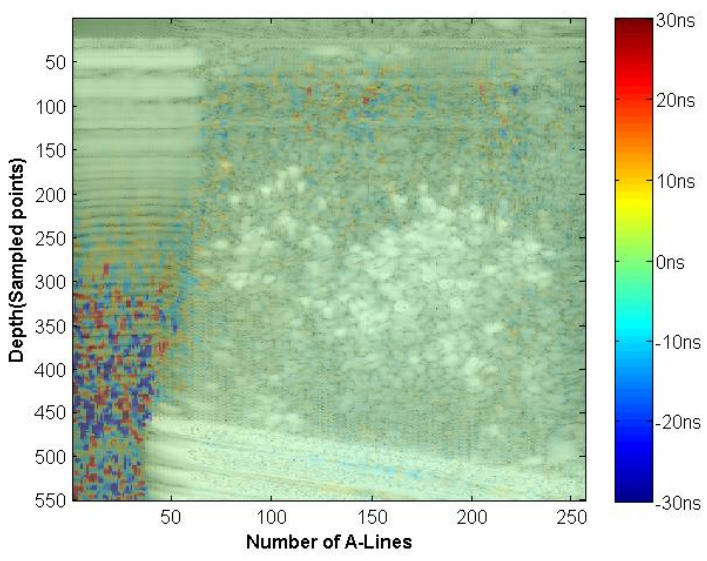

C

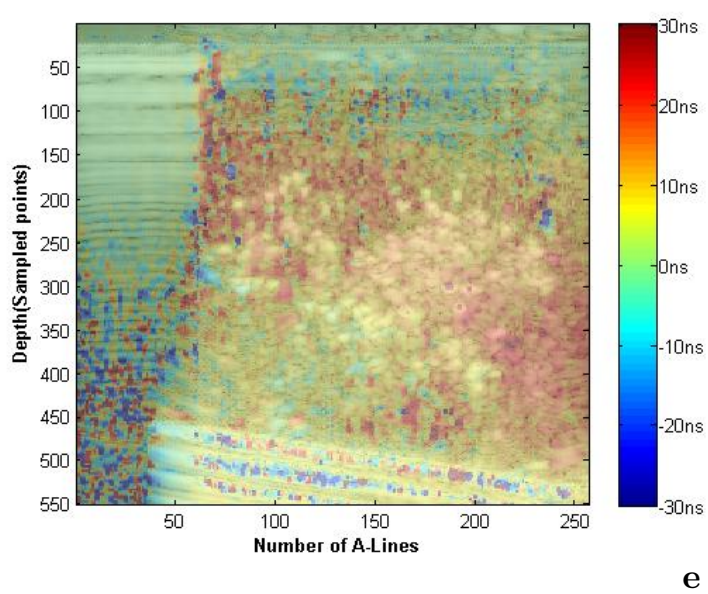

b

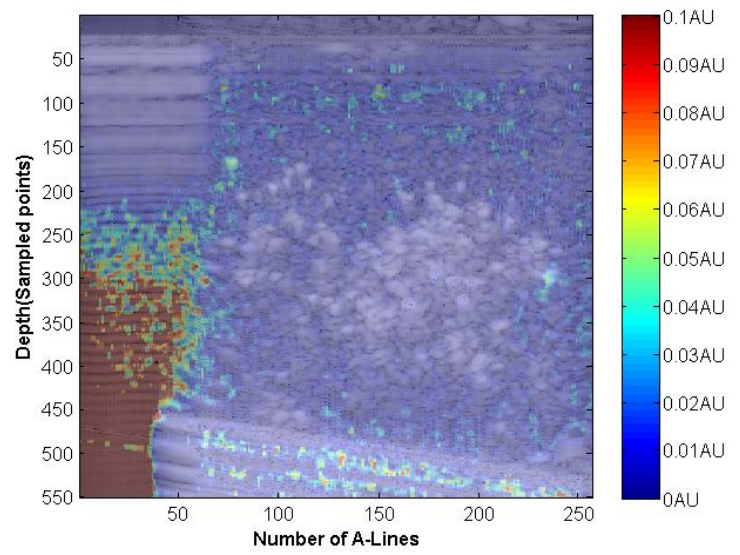

d

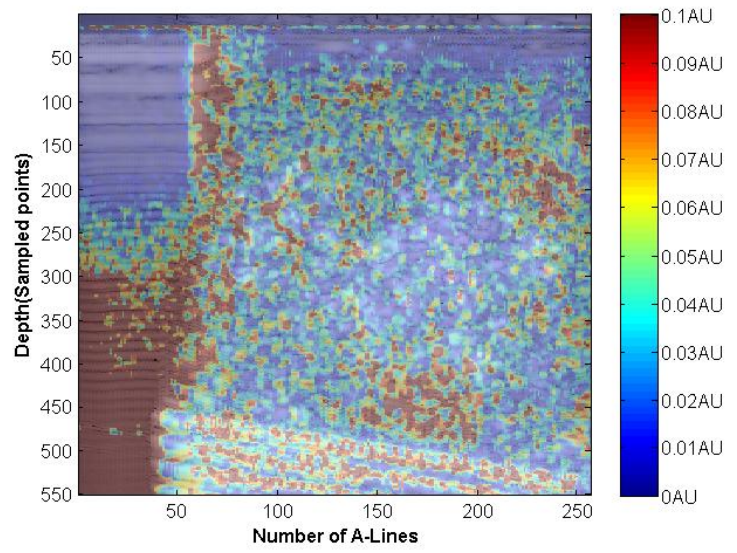

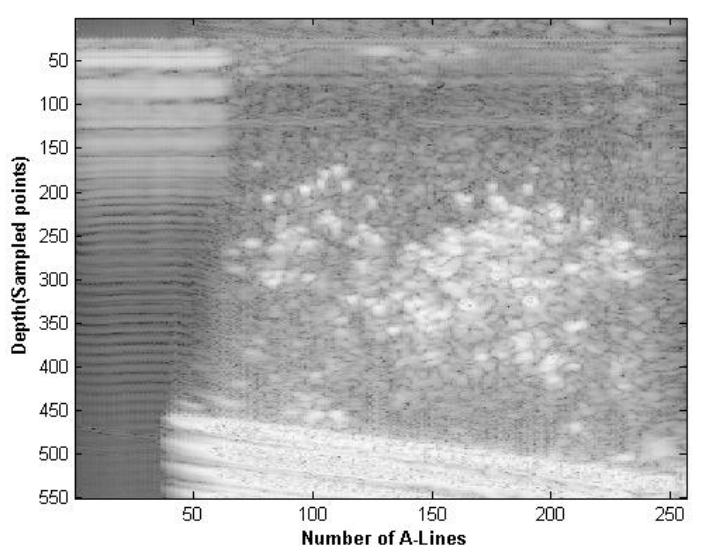

Figure 4.50: Porcine cardiac tissue - Sample III : (a) B-Mode image overlaid with an RGB image generated by the magnitude of the Localised Time Delay Estimates(TDE) before the application of $\vec{E}$ field . (b) B-Mode image overlaid with an RGB image generated by the magnitude of the Localised Amplitude Changes(LAC) before the application of $\vec{E}$ field. (c) TDE overlay after the application of $\vec{E}$ field. (d) LAC overlay after the application of $\vec{E}$ field. (e) B-Mode image of the sample under examination. 
a

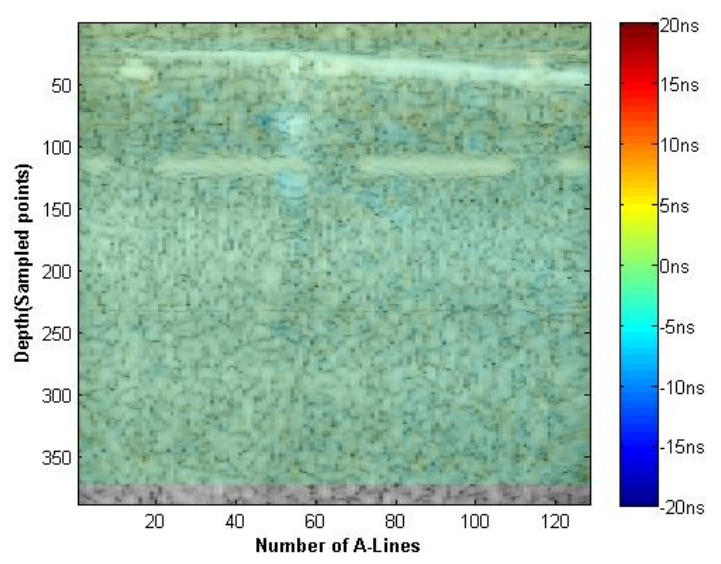

C

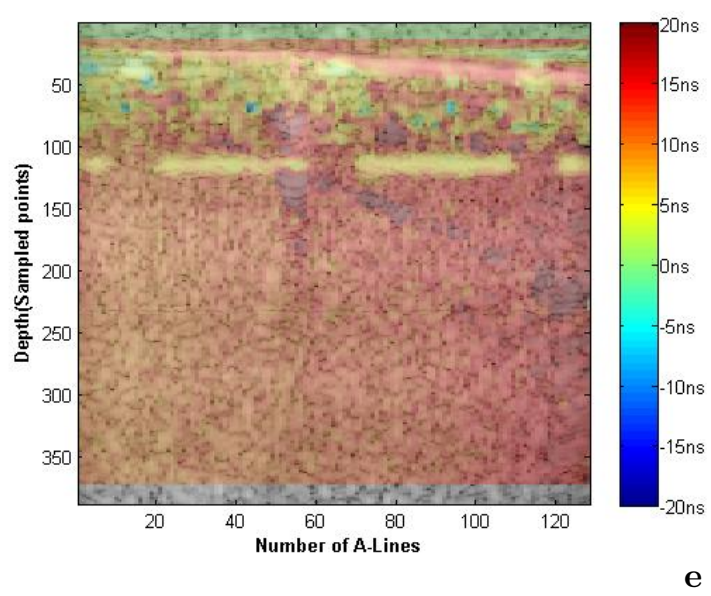

b

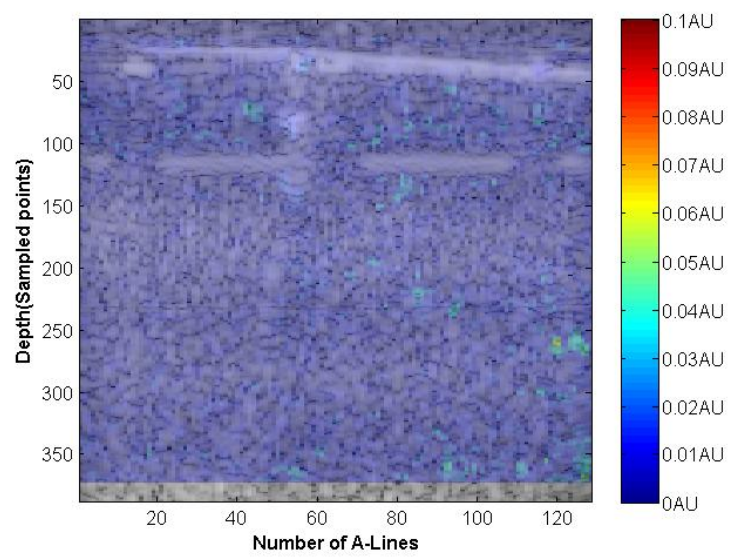

d

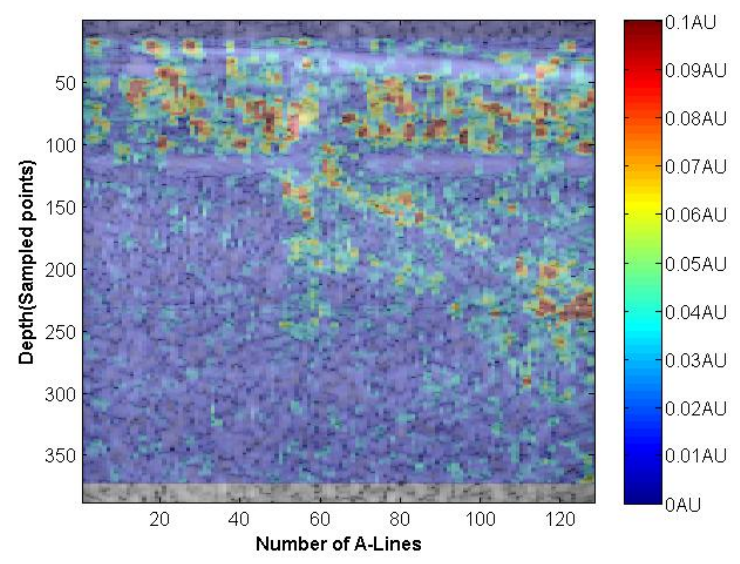

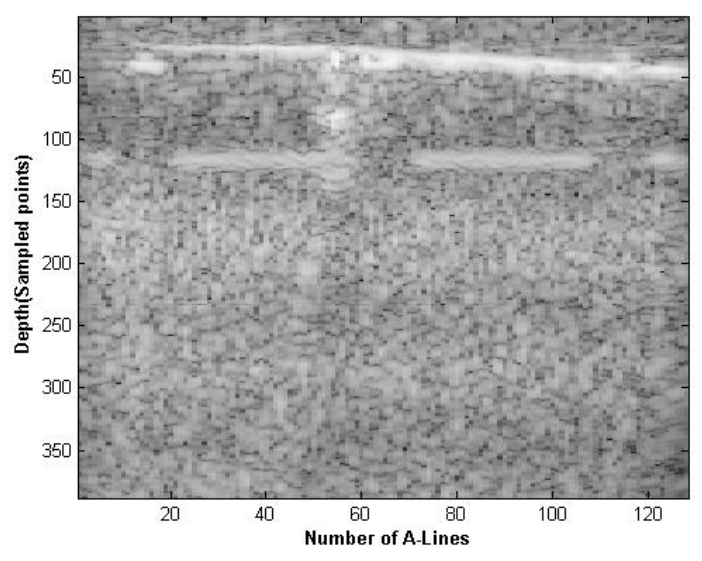

Figure 4.51: Gelatin(6\%) phantom(1\% salt) with graphite(5\%) Phantom-I : (a) B-Mode image overlaid with an RGB image generated by the magnitude of the Localised Time Delay Estimates(TDE) before the application of $\vec{E}$ field . (b) B-Mode image overlaid with an RGB image generated by the magnitude of the Localised Amplitude Changes(LAC) before the application of $\vec{E}$ field. (c) TDE overlay after the application of $\vec{E}$ field. (d) LAC overlay after the application of $\vec{E}$ field. (e) B-Mode image of the sample under examination. 
a

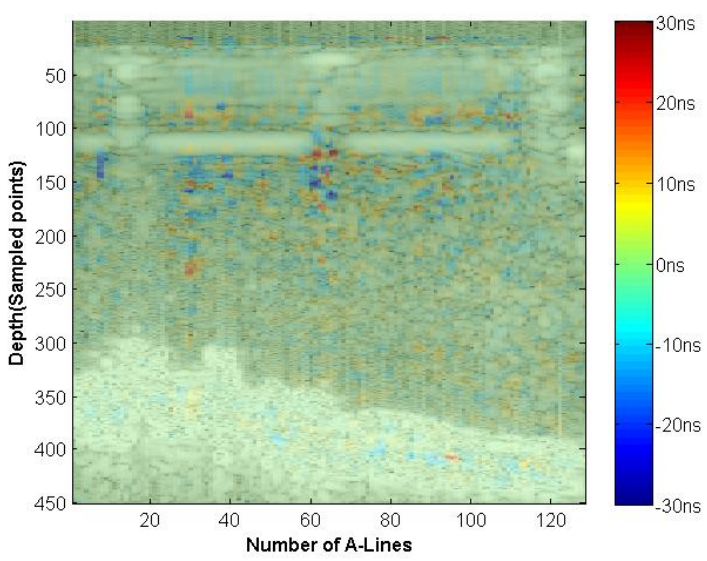

c

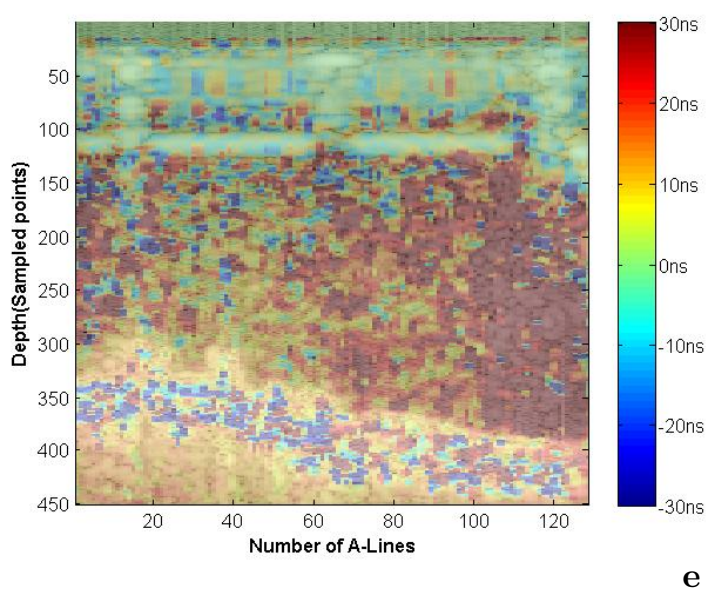

b

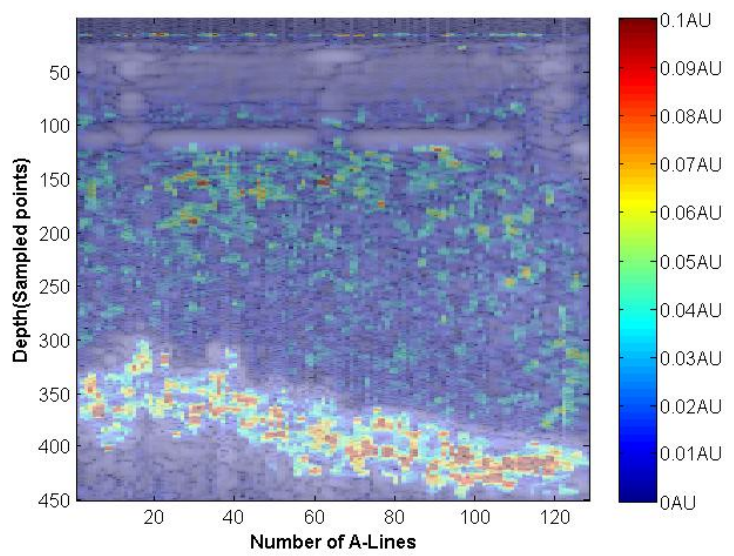

d

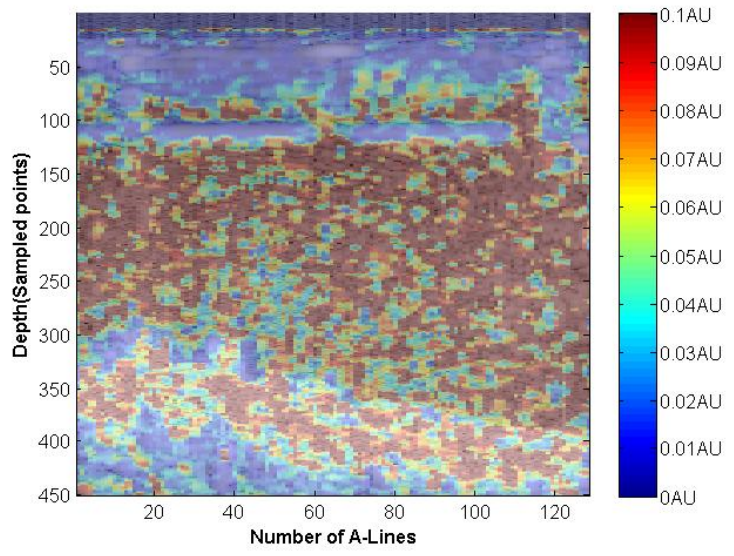

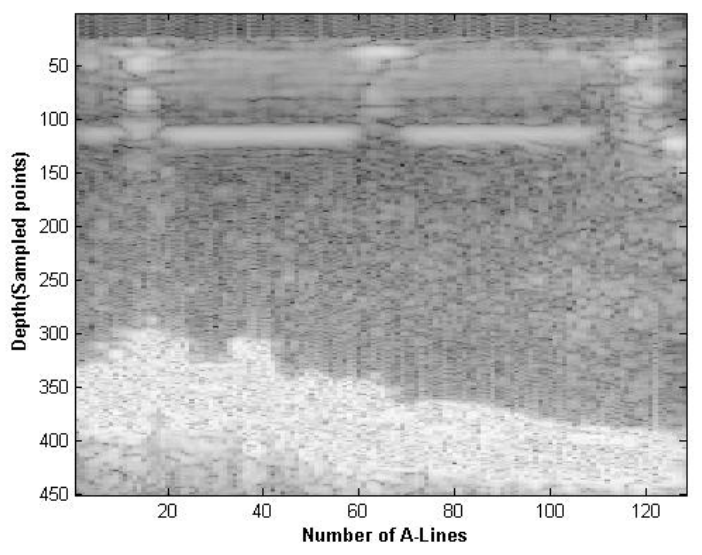

Figure 4.52: Gelatin(6\%) phantom(1\% salt) with graphite(5\%) Phantom-II : (a) B-Mode image overlaid with an RGB image generated by the magnitude of the Localised Time Delay Estimates(TDE) before the application of $\vec{E}$ field . (b) B-Mode image overlaid with an RGB image generated by the magnitude of the Localised Amplitude Changes(LAC) before the application of $\vec{E}$ field. (c) TDE overlay after the application of $\vec{E}$ field. (d) LAC overlay after the application of $\vec{E}$ field. (e) B-Mode image of the sample under examination. 
Chapter 5

\section{Discussion and Conclusions}




\subsection{Discussion}

Phantoms exhibit EIMC which show a dependence on applied electric fields, gelling agent concentration, and salt concentration. EIMC induced in phantoms were found to be depth dependent. Fast-time and slow-time shifting plots indicate that the shifting was higher at the front face of the sample and negligible at the rear face, which shows an expansion of the phantoms. Since the rear boundary of the sample is fixed at the plastic base and the shifting at the rear boundary of the sample is very small, we conclude that the speed of sound in the phantom changes negligibly. The speed of sound depends on the compressibility $\kappa$ and the density $\rho$ of the medium [14. Since the samples were observed to expand along the ultrasound axis direction, it will be interesting to investigate if that expansion results in volume/density changes, which might affect the speed of sound. The magnitude of changes obtained in response to the applied physiological-level electric fields rule out the attribution of these changes to other effects such as piezoelectricity or electrostriction. Temperature measurements indicate that the changes in temperature were insignificant to be considered as a cause for such changes.

Gelling agents formed networked fibres with fixed charges present in protein structure. The application of an electric field to such a network would induce electrokinetic effects such as electro-osmosis, electrophoresis etc. (as described in section 2.6 of this thesis). The combined response of such fixed charges, adsorbed ions, free ions, presence of free and adsorbed water dipoles is evoked by the applied electric fields. The fixed charges in the protein structure (mainly responsible for the interactions with ions) are highly abundant. Various studies on cartilage tissues (special tissues with a high FCD) suggest that the EIMC can be explained by the electro-kinetic behaviour of the tissues [74, 67, 75]. Experiments performed with optical coherence tomography to detect such mechanical changes at the surface of tissues also suggest a similar explanation [19, 20, 21]. It can be concluded that EIMC can be explained by the electro-kinetic behaviour of the phantoms. To our knowledge, the study undertook by us is the first to present images based on the EIMC and such an investigation in soft tissues.

The stiffness increases with the gelling agent concentration at the power of two. The fixed charge densities also increase with the increase in gelling agent concentrations. The dependence of EIMC on gelling agents can be explained by the dependence of the stiffness and FCD on the gelling concentration. It was expected in gelatin phantoms that the rate of change of strain in samples increases with the FCD and decreases with the increasing stiffness. At a low gelatin concentration $(<9 \%$ in Fig. 4.42 , the increase of the FCD dominates the increase of the stiffness, while at high gelatin concentration ( $>9 \%$ in Fig. 4.42, the increase of stiffness dominates the increase of FCD. In agar phantoms, the rate of change of strain decreased with the increase in agar concentration. Since agar forms stiffer gels at low agar concentrations (as opposed to gelatin), it can be suggested that for agar phantoms, the increase of stiffness always dominated the increase of FCD.

An increase in the applied electric field showed an increase in the shifting rate and shifting fluctuations for gelatin phantoms. A linear trend of shifting fluctuations, at the frequency of applied electric field, was observed for $3 \%$ \& $6 \%$ gelatin samples. For $9 \%$ samples, this trend was observed to be exponential. Probably, at lower strain, the dependence of EIMC on voltage is linear while at higher strain the dependence is exponential. For $3 \%$ and $6 \%$ gelatin phantom, the strain in the samples induced by the 
voltage range $(1-4.5$ Volts $)$ is low. For $9 \%$ gelatin phantoms, the strain induced by the same voltage is high, thus has an exponential behaviour. More studies on the voltage dependence are necessary for a better understanding.

Increased salt concentration also increased the EIMC in phantoms. Addition of salt to the phantoms acts in a twofold manner. It increases the conductivity while decreasing the stiffness of gels (due to its protein adsorption and disruption of the hydrogen bonds). Hence, addition of salts to the phantoms increases the EIMC.

The variability of results show that prolonged exposure of the phantom to electric fields led to an erratic response. The same was observed in response to the application of high electric fields(application higher that $4-5 \mathrm{~V})$. This suggests that long time exposure as well as high electric fields result in structural changes within the phantoms.

Constant current and constant voltage sources have been widely used for applying electrical fields to biological media 76, 77, 78]. The proper choice of the source depends on the experimental setup and the materials involved. For electrodermal recording, a constant current source was found to be disadvantageous due to the presence of sweat glands which act as parallel shunts through the epidermal tissue [77, 79, 80]. A constant current source might be a preferred choice in our experiments at it can eliminate the effect of electrode polarization. In this study, a constant voltage source was used for convenience. However, we monitor the electric field in the samples with a voltage probe to ensure that proper level of electric field was applied to the samples. In future, a constant current source can be investigated in more detail.

The fast-time and slow-time shifting results suggest that the EIMC variate locally within different phantoms and are also depth dependent. This was due to the fact that the rear boundary of the samples was fixed. Thus, using a linear array transducer such local variations of EIMC within phantoms and biological tissues were investigated. Cardiac tissues (due to their high conductivity) and layered fat-muscle tissues (to observe EIMC contrast within layered tissues) were chosen for experiments. For cardiac tissues, a contrast was observed via the TDE images and AMP images. The cardiac tissue is a complex structure and the contrast is suggested to be based upon the structural configuration. For a layered tissue, it was found that the contrast generated by EIMC was able to distinguish between the layers of tissues. These changes could also suggest electrophoretic as well as electrosomotic mobility inside the tissue structure. Since these mobilities depend on the FCD, mobile charges and the electrical double layers formed in the tissue structure, local contrast can be obtained based on the electrokinetic nature of the tissues.

For phantoms, the response was more homogeneous when compared to tissues. Fig. 4.52 showed contrast in a layered phantom (where the layer was created by the sedimentation of graphite particles) which also suggests that EIMC have the potential to generate a contrast based on the structure of the sample being investigated. The sampling rate and the signal to noise ratio of the ultrasound system were low in order to observe EIMC and hence a qualitative study could not be performed. 


\subsection{Conclusions and Future-work}

In conclusion, EIMC were observed in various tissue-mimicking ultrasound phantoms as well as tissues. These changes are suggested to be attributed to the electrokinetic behaviour of the samples. The dependence of EIMC were observed to be on the applied electric fields, gelling agent concentration, and salt concentration of the phantoms. Linear trends were observed in shifting fluctuations for $3 \%$ and $6 \%$ gelatin phantoms whereas exponential trends were observed for $9 \%$ gelatin phantoms in response to the applied electric fields. The concentration of gelatin in the phantoms showed an increasing strain rate at lower concentrations ( $<9 \%$ gelatin concentration) and a decreasing strain rate at higher concentrations $(>9 \%$ gelatin concentration).

We also presented that the EIMC could be detected using a linear array transducer. The TDE and AMP images were able to generate contrast and in turn distinguish layers of some underlying tissues and phantoms. Further investigations are required in order to evaluate the potential of using EIMC as an additional contrast method for traditional B-mode imaging.

\section{Future-work}

- Since both the stiffness and FCD are important parameters for EIMC, they should be measured towards a quantitative study of EIMC. The stiffness and FCD are co-dependent on the concentration of gelling agents, hence experiments with a wider range of concentrations of gelling agents could be performed to observe such dependence. The supersonics imaging machine 81] can be used to measure the stiffness of the sample. In that machine, the elasticity of the sample is measured by imaging the radiation-force induced shear-wave propagation. To measure FCD, a two point conductivity approach which includes sample equilibration in two different known concentrations of $\mathrm{KCl}$ as described in 75 . can be followed. More data points in the study on the dependence on salt, gelling agent, voltage can be acquired to understand the effects.

- The frequency of the applied electric fields used in this study was $0.025 \mathrm{~Hz}$. Frequency dependence (below $1 \mathrm{~Hz}$ ) can be pursued which might limit the structural changes occurring after prolonged exposure to electric fields.

- This study included the observation of strain only in the axial direction of the transducer. Lateral and transversal directions could also be pursued to better understand the electrokinetic effects in three dimensions.

- When imaging the EIMC, a limitation was the sampling rate $(\approx 40 \mathrm{MHz})$ and SNR of the ultrasound imaging system. A system with a higher sampling rate and SNR can be chosen for better estimation of TDE and AMP images. Phantoms with layers or inclusions bearing contrasting properties(elastic and electric) can be implemented to observe the EIMC images. Signal-to-noise (SNR) and contrast-to-noise (CNR) ratio could also be investigated in order to evaluate the imaging potential while using such phantoms. 
- After proper characterization, in-vivo experiments could be performed on animal models. Electric current application can be performed in a similar manner as used in electrical impedance tomography. Arrays of electrodes could be used to inject electrical current while constantly monitoring the tissue using ultrasound. Images can be obtained which might provide enhanced contrast based on the electrokinetic nature of tissues. 


\section{References}

[1] T. Szabo, Diagnostic Ultrasound Imaging Inside Out. Saint Louis, MO, USA: Elsevier, Academic Press, 092004.

[2] K. Dussik, "On the possibility of using ultrasound waves as a diagnostic aid," Neurology and Psychiatry, vol. 174, pp. 153-168., 1942.

[3] P. Dijkmans, L. Juffermans, R. Musters, A. van Wamel, F. ten Cate, W. van Gilst, C. Visser, N. de Jong, and O. Kamp, "Microbubbles and ultrasound: From diagnosis to therapy," European Journal of Echocardiography, vol. 5, no. 4, pp. 245-256, 2004.

[4] R. Gramiak and P. Shah, "Echocardiography of the aortic root.," Investigative Radiology, vol. 3, no. 5, pp. 356-366, 1968.

[5] L. Wang, X. Zhao, H. Sun, and G. Ku, "Microwave-induced acoustic imaging of biological tissues," Review of Scientific Instruments, vol. 70, no. 9, pp. 3744-3748, 1999.

[6] Y. Wang, D. Xing, Y. Zeng, and Q. Chen, "Photoacoustic imaging with deconvolution algorithm," Physics in Medicine and Biology, vol. 49, no. 14, pp. 3117-3124, 2004.

[7] A. S. Dukhin and P. J. Goetz, "Acoustic and electroacoustic spectroscopy," Langmuir, vol. 12, no. 18, pp. 4336-4344, 1996.

[8] Y. Xu and B. He, "Magnetoacoustic tomography with magnetic induction (mat-mi)," Physics in Medicine and Biology, vol. 50, no. 21, pp. 5175-5187, 2005.

[9] L. A. Geddes and H. E. Hoff, "The discovery of bioelectricity and current electricity the galvani-volta controversy," Spectrum, IEEE, vol. 8, pp. 38 -46, dec. 1971.

[10] C. McCaig, A. Rajnicek, B. Song, and M. Zhao, "Controlling cell behavior electrically: Current views and future potential," Physiological Reviews, vol. 85, no. 3, pp. 943-978, 2005.

[11] F. of American Societies for Experimental biology and A. P. Society(FASEB), Membranes ions and impulses. 1975. Proceedings of the FASEB.

[12] H. Schwan, "Electrical properties of tissue and cell suspensions.," Advances in biological and medical physics, vol. 5, pp. 147-209, 1957. 
[13] S. Grimnes and O. G. Martinsen, Bioimpedance and Bioelectricity Basics (Biomedical Engineering). Elsevier, Academic Press, second edition ed., 2008.

[14] R. Cobbold, Foundations of biomedical ultrasound. Biomedical Engineering Series, Oxford University Press, 2007.

[15] J. Ophir, I. Cespedes, H. Ponnekanti, Y. Yazdi, and X. Li, "Elastography: A quantitative method for imaging the elasticity of biological tissues," Ultrasonic Imaging, vol. 13, no. 2, pp. 111-134, 1991.

[16] R. P. Henderson and J. G. Webster, "An impedance camera for spatially specific measurements of the thorax," Biomedical Engineering, IEEE Transactions on, vol. BME-25, pp. 250 -254, may 1978.

[17] Staffan and Wall, "The history of electrokinetic phenomena," Current Opinion in Colloid $\mathscr{E}$ amp Interface Science, vol. 15, no. 3, pp. 119 - 124, 2010.

[18] C. Bellmann, A. Caspari, V. Albrecht, T. Loan Doan, E. Mder, T. Luxbacher, and R. Kohl, "Electrokinetic properties of natural fibres," Colloids and Surfaces A: Physicochemical and Engineering Aspects, vol. 267, no. 1-3, pp. 19-23, 2005.

[19] J.-I. Youn, T. Akkin, and T. Milner, "Electrokinetic measurement of cartilage using differential phase optical coherence tomography," Physiological Measurement, vol. 25, no. 1, pp. 85-95, 2004.

[20] J.-I. Youn, T. Akkin, B. Wong, G. Peavy, and T. Milner, "Depth-resolved detection of electrokinetic effects in cartilage using differential phase sensitive optical coherence tomography," vol. 4949, pp. 319-324, 2003.

[21] T. Akkin, D. P. Dav, J.-I. Youn, S. A. Telenkov, H. G. Rylander, and T. E. Milner, "Imaging tissue response to electrical and photothermal stimulation with nanometer sensitivity," Lasers in Surgery and Medicine, vol. 33, no. 4, pp. 219-225, 2003.

[22] O. Doganay and Y. Xu, "Electric-field induced strain in biological tissues," The Journal of the Acoustical Society of America, vol. 128, no. 5, pp. EL261-EL267, 2010.

[23] I. C. on Radiation Units and Measurements, Tissue substitutes, phantoms, and computational modelling in medical ultrasound. International Commission on Radiation Units and Measurements, 1998.

[24] M. O. Culjat, D. Goldenberg, P. Tewari, and R. S. Singh, "A review of tissue substitutes for ultrasound imaging," Ultrasound in Medicine Ėamp Biology, vol. 36, no. 6, pp. 861 - 873, 2010.

[25] C. John, "The corono-apically varying ultrasonic velocity in human hard dental tissues," The Journal of the Acoustical Society of America, vol. 116, no. 1, pp. 545-556, 2004.

[26] E. L. Madsen, M. A. Hobson, H. Shi, T. Varghese, and G. R. Frank, "Tissue-mimicking agar/gelatin materials for use in heterogeneous elastography phantoms," Physics in Medicine and Biology, vol. 50, no. 23, p. 5597, 2005. 
[27] S. H. Oh, B. I. Lee, E. J. Woo, S. Y. Lee, T.-S. Kim, O. Kwon, and J. K. Seo, "Electrical conductivity images of biological tissue phantoms in mreit," Physiological Measurement, vol. 26, no. 2, p. S279, 2005.

[28] T.-J. Kao, G. Saulnier, D. Isaacson, T. Szabo, and J. Newell, "A versatile high-permittivity phantom for eit," Biomedical Engineering, IEEE Transactions on, vol. 55, pp. 2601 -2607, nov. 2008.

[29] K. te Nijenhuis, "Gelatin," in Thermoreversible Networks, vol. 130 of Advances in Polymer Science, pp. 160-193, Springer Berlin / Heidelberg, 1997.

[30] A. Veis, The macromolecular chemistry of gelatin. Molecular biology, Academic Press, 1964.

[31] G. Phillips and P. Williams, Handbook of hydrocolloids. Woodhead Publishing in Food Science and Technology, CRC Press, 2000.

[32] M. Djabourov, "Gelationa review," Polymer International, vol. 25, no. 3, pp. 135-143, 1991.

[33] G. Pollack, Cells, gels and the engines of life: a new, unifying approach to cell function. Ebner \& Sons, 2001.

[34] F. Duck, Physical properties of tissue: a comprehensive reference book. Academic Press, 1990.

[35] K. T. Nijenhuis. PhD thesis, Delft University of technology, Netherlands, 1979.

[36] V. Normand, S. Muller, J.-C. Ravey, and A. Parker, "Gelation kinetics of gelatin: a master curve and network modeling," Macromolecules, vol. 33, no. 3, pp. 1063-1071, 2000.

[37] A. Bot, I. A. van Amerongen, R. D. Groot, N. L. Hoekstra, and W. G. Agterof, "Large deformation rheology of gelatin gels," Polymer Gels and Networks, vol. 4, no. 3, pp. 189 - 227, 1996.

[38] S. Chatterjee and H. Bohidar, "Effect of salt and temperature on viscoelasticity of gelatin hydrogels," Journal of Surface Science and Technology, vol. 22, no. 1-2, pp. 1-13, 2006.

[39] K. te Nijenhuis, "Agarose," in Thermoreversible Networks, vol. 130 of Advances in Polymer Science, pp. 194-202, Springer Berlin / Heidelberg, 1997.

[40] S. Sun, Physical chemistry of macromolecules: basic principles and issues. John Wiley \& Sons, 2004.

[41] M. Watase and K. Nishinari, "Rheological properties of agarose gels with different molecular weights," Rheologica Acta, vol. 22, no. 6, pp. 580-587, 1983.

[42] A. H. Clark, R. K. Richardson, S. B. Ross-Murphy, and J. M. Stubbs, "Structural and mechanical properties of agar/gelatin co-gels. small-deformation studies," Macromolecules, vol. 16, no. 8, pp. 1367-1374, 1983.

[43] C. H. Durney, H. Massoudi, and M. F. lskander, "Radiofrequency radiation dosimetry handbook," tech. rep., University of Utah, 1986. 
[44] O. G. Martinsen and S. Grimnes, Bioimpedance and Bioelectricity Basics, Second Edition. Academic Press, 2 ed., apr 2008.

[45] P. Åberg, Skin cancer as seen by electrical impedance. PhD thesis, Karolinska Institute, Stockoholm, Sweden.

[46] U. G. Kyle, I. Bosaeus, A. D. D. Lorenzo, P. Deurenberg, M. Elia, J. M. Gmez, B. L. Heitmann, L. Kent-Smith, J.-C. Melchior, M. Pirlich, H. Scharfetter, A. M. Schols, and C. Pichard, "Bioelectrical impedance analysispart i: review of principles and methods," Clinical Nutrition, vol. 23, no. 5, pp. 1226 - 1243, 2004.

[47] R. Tregear, Physical functions of skin. Theoretical and experimental biology, Academic Press, 1966.

[48] K. Kontturi, L. Murtomki, J. Hirvonen, P. Paronen, and A. Urtti, "Electrochemical characterization of human skin by impedance spectroscopy: The effect of penetration enhancers," Pharmaceutical Research, vol. 10, pp. 381-385, 1993.

[49] J. Webster and J. Clark, Medical instrumentation: application and design. Houghton Mifflin, 1978.

[50] R. Gudivaka, D. Schoeller, R. Kushner, and M. Bolt, "Single- and multifrequency models for bioelectrical impedance analysis of body water compartments," Journal of Applied Physiology, vol. 87, no. 3, pp. 1087-1096, 1999.

[51] N. Thakor, F. J. J.M., J. Saiz, B. Gramatikov, and J. Ferrero Sr., "Electrophysiologic models of heart cells and cell networks: Enabling a better understanding of arrhythmias and heart disease," IEEE Engineering in Medicine and Biology Magazine, vol. 17, no. 5, pp. 73-83, 1998.

[52] D. Miklavi and F. X. Hart, "Fx: Electric properties of tissues," in In Wiley Encyclopedia of Biomedical Engineering, John Wiley \& Sons, 2006.

[53] A. Guyton and J. Hall, Textbook of medical physiology. Elsevier Saunders, 2006.

[54] E. Neumann, S. Kakorin, and K. Tnsing, "Fundamentals of electroporative delivery of drugs and genes," Bioelectrochemistry and Bioenergetics, vol. 48, no. 1, pp. 3 - 16, 1999.

[55] S. Mthot, V. Moulin, D. Rancourt, M. Bourdages, D. Goulet, M. Plante, F. A. Auger, and L. Germain, "Morphological changes of human skin cells exposed to a dc electric field in vitro using a new exposure system," The Canadian Journal of Chemical Engineering, vol. 79, no. 4, pp. 668-677, 2001.

[56] E. K. Onuma and S. W. Hui, "Electric field-directed cell shape changes, displacement, and cytoskeletal reorganization are calcium dependent.," The Journal of Cell Biology, vol. 106, no. 6, pp. 2067-2075, 1988.

[57] K. Robinson, "The responses of cells to electrical fields: A review," Journal of Cell Biology, vol. 101, no. 6, pp. 2023-2027, 1985. 
[58] M. B. A. Djamgoz, M. Mycielska, Z. Madeja, S. P. Fraser, and W. Korohoda, "Directional movement of rat prostate cancer cells in direct-current electric field," Journal of Cell Science, vol. 114, no. 14, pp. 2697-2705, 2001.

[59] M. Mycielska, M.E. andDjamgoz, "Cellular mechanisms of direct-current electric field effects: Galvanotaxis and metastatic disease," Journal of Cell Science, vol. 117, no. 9, pp. 1631-1639, 2004.

[60] G. Tai, B. Reid, and M. Cao, L. andZhao, "Electrotaxis and wound healing: experimental methods to study electric fields as a directional signal for cell migration.," Methods in molecular biology (Clifton, N.J.), vol. 571, pp. 77-97, 2009.

[61] B. Song, Y. Gu, J. Pu, B. Reid, Z. Zhao, and M. Zhao, "Application of direct current electric fields to cells and tissues in vitro and modulation of wound electric field in vivo," Nature Protocols, vol. 2, no. 6, pp. 1479-1489, 2007.

[62] J. D. DeNuzzio and B. Berner, "Electrochemical and iontophoretic studies of human skin," Journal of Controlled Release, vol. 11, no. 1-3, pp. 105 - 112, 1990. Proceedings of the Fourth International Symposium on Recent Advances in Drug Delivery Systems.

[63] A. K. Banga and Y. W. Chien, "Iontophoretic delivery of drugs: Fundamentals, developments and biomedical applications," Journal of Controlled Release, vol. 7, no. 1, pp. 1-24, 1988.

[64] A. K. Banga, S. Bose, and T. K. Ghosh, "Iontophoresis and electroporation: comparisons and contrasts," International Journal of Pharmaceutics, vol. 179, no. 1, pp. 1 - 19, 1999.

[65] A. Delgado, F. Gonzlez-Caballero, R. Hunter, L. Koopal, and J. Lyklema, "Measurement and interpretation of electrokinetic phenomena," Journal of Colloid and Interface Science, vol. 309, no. 2, pp. 194 - 224, 2007. Elkin 06, International Electrokinetics Conference, June 25-29, Nancy, France.

[66] M. Aumailley and B. Gayraud, "Structure and biological activity of the extracellular matrix," Journal of Molecular Medicine, vol. 76, pp. 253-265, 1998.

[67] V. Mow, G. Ateshian, W. Lai, and W. Gu, "Effects of fixed charges on the stress-relaxation behavior of hydrated soft tissues in a confined compression problem," International Journal of Solids and Structures, vol. 35, no. 34-35, pp. 4945-4962, 1998.

[68] H. Ohshima, "Electrokinetic phenomena in a concentrated suspension of soft particles," Colloids and Surfaces A: Physicochemical and Engineering Aspects, vol. 195, no. 1-3, pp. 129-134, 2001.

[69] D. A. Saville, "Electrokinetic effects with small particles," Annual Review of Fluid Mechanics, vol. 9, no. 1, pp. 321-337, 1977.

[70] J. C. Baygents, "Electrokinetic effects on the dielectric response of colloidal particles: Dielectrophoresis and electrorotation," Colloids and Surfaces A: Physicochemical and Engineering Aspects, vol. 92, pp. 67-77, 1994. 
[71] K. Stana-Kleinschek and V. Ribitsch, "Electrokinetic properties of processed cellulose fibers," Colloids and Surfaces A: Physicochemical and Engineering Aspects, vol. 140, no. 1-3, pp. 127-138, 1998.

[72] P. Irena, P. Tanja, L. Thomas, B. Darinka, C. Simona, and P. Romana, "Elektrokinetic studies of raw and modified cotton fabrics," Book of Proceedings of the 4th International Textile, Clothing and Design Conference - Magic World of Textiles, pp. 864-869, 2008.

[73] C. Bellmann, A. Caspari, V. Albrecht, T. Loan Doan, E. Mder, T. Luxbacher, and R. Kohl, "Electrokinetic properties of natural fibres," Colloids and Surfaces A: Physicochemical and Engineering Aspects, vol. 267, no. 1-3, pp. 19-23, 2005.

[74] E. H. Frank and A. J. Grodzinsky, "Cartilage electromechanicsi. electrokinetic transduction and the effects of electrolyte ph and ionic strength," Journal of Biomechanics, vol. 20, no. 6, pp. 615 $627,1987$.

[75] A. Jackson, T.-Y. Yuan, C.-Y. Huang, and W. Gu, "A conductivity approach to measuring fixed charge density in intervertebral disc tissue," Annals of Biomedical Engineering, vol. 37, pp. 2566$2573,2009$.

[76] W. Greatbatch, B. Piersma, F. D. Shannon, and S. W. Calhoon, "Polarization phenomena relating to physiological electrodes," Annals of the New York Academy of Sciences, vol. 167, no. 2, pp. 722$744,1969$.

[77] F. Schaefer and W. Boucsein, "Comparison of electrodermal constant voltage and constant current recording techniques using the phase angle between alternating voltage and current," Psychophysiology, vol. 37, no. 1, pp. 85-91, 2000.

[78] D. Linden and T. Reddy, Handbook of batteries. McGraw-Hill handbooks, McGraw-Hill, 2002.

[79] C. Brown, Methods in psychophysiology. Williams \& Wilkins, 1967. pp. 153.

[80] A. Ross, G. Saulnier, J. Newell, and D. Isaacson, "Current source design for electrical impedance tomography," Physiological Measurement, vol. 24, no. 2, pp. 509-516, 2003.

[81] J. Bercoff, M. Tanter, and M. Fink, "Supersonic shear imaging: a new technique for soft tissue elasticity mapping," Ultrasonics, Ferroelectrics and Frequency Control, IEEE Transactions on, vol. 51, pp. $396-409$, april 2004. 